\title{
Evolutionary PTEN gene divergence underpins the remodeling of plant vacuolar compartments
}

Bojan Gujas ${ }^{1 *}$, Chloe Champeyroux ${ }^{1}$, Anna Hunkeler ${ }^{1}$, Emilija Robinson ${ }^{1}$, Noel Blanco-Touriñán ${ }^{1}$, Tiago Miguel Dias Cruz ${ }^{1}$, Matthias Van Durme ${ }^{2,3}$, Moritz K. Nowack $^{2,3}$, Antia Rodriguez-Villalon ${ }^{1 *}$

${ }^{1}$ Institute of Molecular Plant Biology, Department of Biology, Swiss Federal Institute of Technology (ETH) Zurich, Zurich, CH-8092, Switzerland.

2Department of Plant Biotechnology and Bioinformatics, Ghent University, 9052 Ghent, Belgium

3VIB-UGENT Center for Plat Systems Biology, 9052 Ghent, Belgium

*Correspondence: bojan.gujas@biol.ethz.ch, antia.rodriguez@biol.ethz.ch

\section{Summary}

Membrane fusion and fission are fundamental processes in sustaining cellular compartmentalization. Fission of a lipid bilayer requires a furrow formation that brings membranes in close proximity prior to a contiguous membrane cleavage. Although plant ancestors abandoned cleavage furrow-mediated cytokinesis more than 500 million years ago, here we show that plants still employ this mechanical principle to divide embryonic vacuoles. The evolutionary divergence in PHOSPHATASE AND TENSIN HOMOLOG DELETED ON CHROMOSOME TEN (PTEN) enzymes was required to coordinate this process, as Arabidopsis loss-of-function pten $2 a$ pten $2 b$ mutants contain hyper compartmentalized embryonic vacuoles. In contrast, PTEN2 overexpression hinders lytic and secretion cellular pathways downstream of TGN in xylem cells. These processes are critical for the formation of secondary cell walls in xylem cells and depend on a poorly characterized and evolutionarily novel $\mathrm{N}$-terminal domain in PTEN2s. The PTEN2 subfamily appeared with the emergence of the Phragmoplastophyta clade, when vacuolar compartments enlarged and cleavage furrow-mediated cytokinesis became extinct. Together, our work suggests that the evolutionary innovation of the PTEN family is conserved across terrestrial plants and central to vacuolar remodelling.

Keywords: Vacuole division, cleavage furrow, xylem, cell differentiation, PTEN 


\section{INTRODUCTION}

Cell compartmentalisation is a basic organisational principle of eukaryotic life that separates variety of partitions within the cell to generate multiple different metabolic environments. Communication between endomembrane compartments occurs by a tightly regulated interplay of membrane fission and fusion ${ }^{1,2}$. Although the molecular players involved in membrane fission can vary across living kingdoms, a common mechanistic requirement is to bring two membranes into close proximity ${ }^{1,3}$. Generally, this energetically costly membrane bending is followed by a furrow formation at the cleavage site that progresses centripetally until a neck structure is generated on which cleavage proteins can act ${ }^{3,4}$. The membrane bending requires dynamic changes in its physiochemical properties. For example, the activation of phosphatidylinositol 3kinase (Ptdlns3-kinase) to produce phosphatidylinositol 3,4,5-trisphosphate (PtdIns[3,4,5]P 3 ) was shown to be a prerequisite for membrane ruffling in mammalian cells as well as for the leading membrane bending during neutrophil cells or amoeba chemotaxis ${ }^{5,6}$. Membrane relaxation is achieved by Ptdlns $[3,4,5] \mathrm{P}_{3}$ dephosphorylation at the 3' position by PHOSPHATASE AND TENSIN HOMOLOG DELETED ON CHROMOSOME TEN (PTEN) activity from the rear sides of the motile membrane ${ }^{7}$. Interestingly, the phosphoinositides species phosphorylated at 3' positions are scarce in plants, while Ptdlns[3,4,5]P 3 has never been detected ${ }^{8,9}$. Yet, the Arabidopsis thaliana (Arabidopsis) genome encodes three PTEN homologs split in two subfamilies: PTEN1, and PTEN2 comprised of the paralogs PTEN2a and PTEN2b (PTEN2s $)^{10}$. The need for expansion of PTEN genes in plants is unclear to date.

In the green lineage, cytokinesis mechanism evolved from ancestral centripetal cleavage, still occurring in algal Streptophyta clade, to centrifugal fusion model where vesicles fuse to the growing septum membrane called cell plate ${ }^{11,12}$. Membrane donors here are guided by the evolutionary novel structure - phragmoplast, thus all organisms using this model of cytokinesis are collectively termed Phragmoplastophyta, including algal division Charophyta as well as all land plants ${ }^{12}$. In addition to discontinuation of a cleavage furrow for cytokinesis, plant cells enlarged their vacuolar compartment in comparison to unicellular green algae in the protist clade ${ }^{13}$. These large compartments became essential for land plant cells' viability as they provide plant hydrostatic skeleton in a form of turgor pressure ${ }^{14}$. Additionally, vacuoles display a vast array of cellular functions ${ }^{13}$. Vegetative tissues contain lytic vacuoles, which are acidic 
compartments abundant in hydrolases critical for ion homeostasis and lytic degradation $^{13}$. In contrast, protein storage vacuoles (PSVs) accumulate protein reserves that fuel plant development during germination and are characterized by a neutral $\mathrm{pH}^{13}$. Recently, PTEN2a was implicated in vacuolar trafficking in Arabidopsis ${ }^{15}$ - consistent with the reported PTEN2s substrate preference to be Ptdlns3P, typically residing in membranes of lytic compartments ${ }^{9,10,15}$. The latter poses the question whether plant $P$ TEN2s may have a role in remodelling vacuolar membrane (tonoplast) instead of the plasma membrane as reported in other eukaryotes. However, how voluminous vacuolar compartments divide in plants is still a matter of debate ${ }^{16}$. Recent $3 \mathrm{D}$ models of vacuoles suggested that the compartments that appear fragmented in 2D images are actually not physically separated but rather form a tubular network ${ }^{17-19}$. Thus, many previously reported examples of a vacuole fragmentation have to be revalidated in order to gain better understanding of vacuolar membrane dynamics.

Here, we followed the stepwise conversion of large embryonic vacuoles (EVs) into smaller PSVs in Arabidopsis. Our 3D reconstructions of EV remodelling revealed vacuolar division by a process morphologically resembling the progressing cleavage furrow division. Moreover, we show that PTEN2 enzymes are essential to coordinate membrane tubularization at EV division initiation sites and cleavage furrow progression. In pten2a pten $2 b$ double mutants EVs become hyper-compartmentalized instead of fragmented. On the contrary, overexpression of PTEN2s prevents the fusion of trans-Golgi network (TGN)-derived small vacuoles to the central vacuole that does not enlarge but stays tubular. This phenomenon was cell type specific and predominantly occurring in xylem tissues. Aberrant cell trafficking affected both vacuolar and secretory pathways, essential for xylem tissue maturation. Notably, PTEN2 function in remodelling vacuolar architecture depends on their poorly characterized N-terminal domain, that evolutionarily appeared in the Phragmoplastophyta clade, coinciding with vacuolar enlargement and loss of cleavage furrow-mediated cytokinesis ${ }^{20}$. Thus, it seems plausible that PTEN2s evolved to provide molecular support to preserve this ancient model of membrane fission to modulate the biggest plant cell compartment - the vacuole. 


\section{RESULTS}

\section{Embryonic vacuole division involves cleavage furrow formation and requires PTEN2 activity}

During plant embryogenesis, EV become transformed into numerous small PSVs prior seed desiccation ${ }^{21,22}$. To assess whether this process entails vacuolar fragmentation, we followed the dynamics of previously described vacuole marker TONOPLAST INTRINSIC PROTEIN (TIP) 3;2 during Arabidopsis embryo development. Expressed from a native TIP3;2 promoter fragment, this marker is detectable from the late heart or early torpedo stage of Arabidopsis embryogenesis onwards. TIP3;2-GFP first accumulates in the endoplasmic reticulum (ER) around the nucleus and near the plasma membrane, similar to the V-PPase VHP1/AVP1 tonoplast marker at the same stage (stage I in Fig. 1a Extended Data Fig. 1a). In succeeding stages of embryo development, the first pre-EV can be observed in addition to the ER signal (stage II). Next, the TIP3;2 accumulation becomes restricted to the tonoplast of pre-EVs that undergo fusion, evident by the hollowed spaces bridging two vacuoles at the fusion site (stage III in Fig. 1a and Extended Data Fig. 1b). As a result of these homotypic fusions, larger EVs are generated (stage IV in Fig. 1a), followed by the onset of the typical PSV autofluorescence in the successive stages. Next, the large EV starts dividing, evidenced by the symmetrical tonoplast invaginations towards the vacuolar lumen (stage $\mathrm{V}$ in Fig. 1a). By performing 3D reconstructions based on maximal projections and surface rendering we could observe formation of a cleavage furrowlike structure in the region where the tonoplast is contracting around the incipient separation site (Fig. 1b). As one EV splits in multiple PSVs, various cleavage progression stages can be observed in a single EV. Stage VI is characterized by the final fragmentation of EV into multiple PSVs (Fig. 1a). During the process of EV division in some samples we noticed the presence of small vacuoles that may be the membrane source necessary for tonoplast invagination during furrowing or suggest an additional alternative pathway of vacuole fragmentation (Extended Data Fig. 1c).

To determine the molecular mechanisms underpinning embryonic vacuolar division in plants, we decided first to explore whether Ptdlns3P metabolic enzymes are involved in the regulation of this process. In budding yeast, a local enrichment of Ptdlns3P at the neck occurs before membrane fission and it is required to stabilize membrane 
invaginations $^{23}$. Detailed examination of pten2a pten $2 b$ mutants revealed a crucial difference in the progression of EV division in stage $V$ in comparison to wild type embryos (Fig. 1a). In pten2a pten2b embryos the tonoplast inward invaginations are not coordinated, and often asymmetric unilateral. The invaginated membrane can be seen in 3D as individual sheets growing inwards; and even when these sheets meet the other side of the vacuole, a ring-shaped cleavage furrow-like structure will be absent or incomplete (Fig. 1b). The membrane sheet would in later stages roll in on itself and form a cylindrical shape, or sometimes a sphere that may eventually pinch off. At the end, instead of fragmented, EVs of pten2a pten2b mutants appear hypercompartmentalized with their vacuolar lumen crisscrossed with several membranes (stage VI in Fig. 1a). During seedling germination, the homotypic fusion of PSVs (stage VII in Fig. 1a and 1c) generates the central lytic vacuolar compartment, a process that can be scored by the gradual disappearance of the typical PSV autofluorescence by 48h after seed imbibition. Surprisingly, lytic vacuole formation appeared unaffected in pten $2 a$ pten $2 b$ double mutants, although mutant seedlings germinated slightly faster than wild type (Fig. 1d and Extended Data Fig. 1d). Yet, this process does not translate in a faster post-embryonic growth, as manifested by a similar root growth and underlying meristematic activity (Fig. 1e-f).

\section{PTEN2s overexpression prevents xylem cell differentiation}

To further elucidate the potential role of PTEN2s in vacuolar remodeling and its effects on plant development, we decided to analyze the impact of altered PTEN2 levels. As, the constitutive PTEN2b overexpression from UBQ10 promoter was lethal, we employed the estradiol inducible system to assess its short-term overexpression effects in relevant cell types (Extended Data Fig .1e). During germination, the consumption of PSV reserves was proposed to occur primarily in vascular cells, most likely to allow for the development of a functional vascular system before the amino acids are mobilized from other parts of the plant body ${ }^{24,25}$. Hence, we focused on xylem cells. To become conductive units, xylem cells undergo a complex developmental process that encompasses the reinforcement of the cell wall (SCW) and a vacuolardriven programmed cell death $(P C D)^{26,27}$. The formation of fully differentiated xylem cells with lignin-reinforced SCWs can already be observed $48 \mathrm{~h}$ after seed imbibition (Extended Data Fig. 1f). While pten2a pten2b double mutants showed neither 
acceleration nor delay in xylem vessel differentiation, seedlings overexpressing PTEN2b showed significant defects in xylem maturation (Extended Data Fig. 1f). Interestingly, the xylem differentiation inhibitory effect had only overexpression of PTEN2 paralogs but not PTEN1 isoform (Fig 2a-b), suggesting a functional evolutionary divergence between orthologs. In the further text we will mainly focus on PTEN2b overexpressing lines (PTEN2box), as the results obtained in either PTEN2a or PTEN2b overexpressing lines were very redundant. The phenotypical effects of PTEN2 ox were dosage dependent and sensitive to the duration of induction (Extended Data Fig. $1 \mathrm{e}$ and $1 \mathrm{~g}-\mathrm{h})$. The induction with a lower estradiol concentration $(0.2 \mu \mathrm{M})$ for shorter time (24-48h) did not affect much the overall root growth, but inhibited xylem differentiation completely or allowed only individual cells to differentiate (xylem islands) (Fig 2b). Higher expression detected in independent transgenic lines or achieved by estradiol induction in higher concentrations $(2 \mu \mathrm{M})$ significantly shortened the root length but reverted xylem phenotypes almost to normal (Extended Data Fig. 1e and $1 \mathrm{~g}-\mathrm{h})$. The latter was scored mainly in distal root parts where the tissue was longer exposed to PTEN2b induction agent prior differentiation.

Next, we assessed if xylem differentiation in PTEN2 $b_{o x}$ was only delayed or lastingly inhibited by analyzing the expression of known xylem markers associated with xylem maturation. The expression of the protoxylem master regulator VASCULAR RELATED NAC-DOMAIN PROTEIN 7 (VND7) and its downstream target gene MYB DOMAIN PROTEIN 46 (MYB46) in seemingly non-differentiated xylem cells in PTEN2box validated that these cells committed to the xylem cell fate (Fig. 2c). Moreover, the expression of secondary cellulose synthetic machinery subunits as well as PCDassociated enzymes as a hallmark of xylem cell maturation can be detected in PTEN2box (Fig.2c and Extended Data Fig. 2a). These findings demonstrated that although the xylem cells reached their transcriptional maturity, they failed to lay down SCWs as confirmed by transmission electron microscopy (Fig. 2d). Notably, xylem cells failing to form SCWs contained vacuoles of altered morphology compared to wild type (Fig. 2d), implying correlation between the SCW formation and vacuolar morphology in xylem cells. 


\section{PTEN2b overexpression modulates vacuolar and secretory vesicular trafficking of xylem cells}

Despite its key role in protoxylem differentiation ${ }^{27}$, very little is known about xylem vacuolar biogenesis, mostly due to its relatively deep positioning within a root. Hence, we decided first to reassess vacuolar biogenesis in protoxylem developing cells by monitoring VHP1-GFP dynamics. Live-cell imaging, followed by 3D image reconstruction revealed the formation of elongated tubular structures in dividing meristematic cells (Fig. 3a). The subsequent enlargement of tubular compartments results from the fusion of small rounded vacuoles, giving rise to two large vacuolar compartments connected by a narrow tubular connection. PTEN2s ox effectively abolished the fusion of small vacuoles to the elongated tubular vacuole (Fig. 3b-c). Surprisingly, neither PTEN2a $a_{o x}$ nor PTEN2 $b_{o x}$ affected vacuolar morphology in root ground tissues (Fig. 3c). The latter suggested that xylem cells require a cell type specific vacuolar regulation. To corroborate the prevention of the xylem tubular vacuole enlargement and its globularization in PTEN2sox, we assessed the trafficking pathways summarized in Figure 4a. Similar to VHP1, VHA-a3 was present on the membranes of both tubular and small vacuoles in PTEN2 $b_{o x}$ suggesting that the direct ER-to-vacuole trafficking pathway is functional (Fig. 4b). Next, we aimed to assess TGN-dependent vesicle delivery to the vacuole by analyzing an artificial cargo TolM $^{28}$

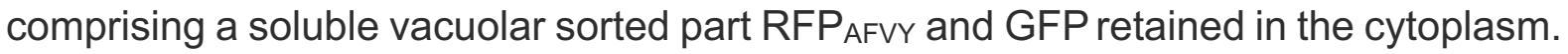
Overexpression of PTEN2b precluded the loading of RFPAFVY into the vacuole, as well as other cargos known to be transported to the vacuole (Fig. 4c-e and Extended Data Fig. 4a). Together, these observations indicated that PTEN2b regulates vesicle trafficking from TGN to the vacuole. This result was consistent with the previously reported PTEN2a localization at $T_{G N}{ }^{15}$. Similarly, PTEN2b exhibits an early colocalization with membrane tracer FM4-64 and TGN marker VHA-a1 (Extended Data Fig. 4b-c). However, failed vacuolar delivery of these cargos cannot directly explain the inability of xylem cells to build a SCW. With the onset of SCW synthesis, the primary cell wall CELLULOSE SYNTHASE (CESA) enzyme complexes ceased to be delivered to the plasma membrane and are gradually removed ${ }^{29}$. As CESA6 is a primary cell wall CESA subunit, it may be plausible to hypothesize that the sequestering of this subunit in the vacuole is critical for the secondary cellulose synthase complex to assemble and/or be active. Although CESA6 colocalized with 
PTEN2b in discrete punctae, PTEN2b overexpression still abolished the xylem SCW formation in cesa6 genetic background (Extended Data Fig. 4d-e), invalidating our hypothesis. SCW formation however also depends on the cellular secretion pathway as hemicellulose, lignin monomers and biosynthetic enzymes must be delivered to the apoplast before crosslinking ${ }^{30-32}$. For example, LACCASE 17 (LAC17) is an enzyme essential for lignin biosynthesis that in wild type plants can be found in the apoplast following the SCW spiral pattern ${ }^{33}$. Surprisingly, in PTEN2box LAC17 was not secreted, but rather retained inside the cell forming aggregates between the vacuoles (Fig. $4 \mathrm{f}$ g), explaining the lack of lignin formation as a part of xylem SCW in PTEN2 $b_{\text {ox. }}$ Although the vacuolar and secretion trafficking pathways may converge at multivesicular-bodies (MVBs), CESA6 and LAC17 aggregates created in PTEN2box did not always colocalize (Extended Data Fig. 4f). The latter suggests that PTEN2b can affect MVBs (as confirmed by Rha1 marker line) but also it may affect TGN downstream pathways at different levels (Extended Data Fig. 4g). In stronger PTEN2box we even occasionally noticed in epidermal cells RABG3f (RAB7 GTPase HOMOLOG) positive aggregates in a grape-like structures seemingly unable to fuse (Extended Data Fig. $4 \mathrm{~h}$ ), further supporting our findings in xylem tissue.

Since vacuolar-driven PCD is the final step of xylem tissue maturation, we evaluated PCD execution in xylem cells upon PTEN2b overexpression. Remarkably, PCD execution can still be detected in cells uncapable of forming SCW, as manifested by the absence of organelles such as the nuclei (Fig. 4h). Xylem cells incapable to form SCW ultimately collapse, as seen in orthogonal sections stained with toluidine blue and transmission electron microscopy images (Fig. 4i-j).

Interestingly, a sharp quenching of YFP signal in comparison to mCHERRY could be detected prior to PCD execution (Fig. 4k-I). The different $\mathrm{pH}$ fluorescence of YFP and mCHERRY proteins ${ }^{34}$ may be accounted for this phenomenon, suggesting timed vacuolar acidification only in the last steps of xylem differentiation. The visibility of YFP fluorophore inside xylem vacuoles suggests that these vacuoles have a milder $\mathrm{pH}^{35}$, similar to storage vacuoles. Hence, this phenomenon may explain the cell-specificity of PTEN2s action on vacuoles in xylem tissues but not in other cell types (Fig.3c).

Together, our results showed that PTEN2s overexpression restricts tubular xylem vacuoles from enlarging. This phenotype is opposite to the phenomenon occurring during EV division, where even membrane tubularization is essential to create a furrow surrounding the incipient cleavage site (Extended Data Fig. 4i). 


\section{PTEN2 function was conserved through evolution before vascular plants appearance}

Contrary to PTEN2s, overexpression of PTEN1 did not affect vacuolar formation nor xylem differentiation (Figs. $2 a-b$ and $3 b-c$ ). This observation raised the question whether the duplication of PTEN enzymes and their vacuolar remodeling function was an evolutionary prerequisite that contributed to the emergence of vascular plants (Fig. 5a). To answer this question, we applied a phylogenomic strategy for identifying orthologs in the green lineage. We blasted Arabidopsis PTEN2b full-length amino acid sequence against proteome assemblies of 142 plant species from chlorophytes to angiosperms (Fig. 5b, Supplementary Table 1). Detailed analysis revealed that PTEN enzymes from the green lineage can be divided into 3 subfamilies: algal PTEN, PTEN1 and PTEN2 (Fig. 5b). Algal PTEN subfamily includes Chlamydomonas reinhardtii PTEN (CrPTEN) that interestingly clusters with the referent human (HsPTEN) isoform. PTEN1 subfamily contains previously mentioned Arabidopsis PTEN1 (AtPTEN1), while PTEN2 subfamily clusters Arabidopsis isoforms (AtPTEN2a and AtPTEN2b) with Marchantia polymorpha PTEN2 isoform (MpPTEN2). The divergence of the PTEN2 gene subfamily could be traced back to the origin of the Phragmoplastophyta clade (Fig. 5a) as confirmed by the absence of xylem and vacuolar phenotypes when overexpressing CrPTEN in Arabidopsis seedlings (Fig. 5c-d). Moreover, we observed that PTEN2 genes conserved their functions even in the non-vascular plant Marchantia as the effect of overexpressing MpPTEN2 mimicked AtPTEN2b overexpression. These observations suggest that vacuolar remodeling and xylem differentiation are PTEN2-specific functions that remained highly conserved across land plants despite hundreds of millions of years of evolution ${ }^{36}$.

\section{PTEN2 function lies in its $\mathbf{N}$-terminal domain that determines its subcellular localization}

Cross-examination of PTEN sequences from the three subfamilies revealed extended $\mathrm{N}$-terminal and C-terminal sequences in PTEN2s compared to other two subfamilies (Fig. 6a). To test whether these sequences may explain PTEN2s functional divergence from other PTEN subfamilies, we overexpressed N-terminal and C-terminal truncated versions of AtPTEN2b (PTEN2 $b^{\Delta N \text { ter }}$ and PTEN2 $b^{\Delta C t e r}$, respectively). Deletion of 
PTEN2b C-terminal sequence did not alter PTEN2b -dependent suppression of xylem continuity, whereas the lack of $\mathrm{N}$-terminal sequence (PTEN2b $\Delta$ Nter) inhibits this enzyme's ability to impair xylem development (Fig. 6b) or vacuolar biogenesis (Fig. 6c). Furthermore, we were able to pinpoint the functional necessity of 57 AA of PTEN2 $\mathrm{N}$-terminal domain (PTEN2 $\mathrm{b}^{\Delta 1-131}$ ) upstream of its phosphatase catalytic domain (Fig. 6a-b). This conserved N-terminal part of PTEN2s makes it less hydrophobic in comparison to N-terminal of PTEN1, supporting the functional divergence between orthologues (Extended Data Fig. 6a-b). As expected, the N-terminals swapping from PTEN1 to PTEN2b (N1-PTEN2b) abolished PTEN2b function (Fig. 6b), confirming the functional specificity of PTEN2 N-terminus. Further in silico mining pointed out an enrichment in intrinsically disordered domains in the PTEN2 N-terminus suggesting the importance of macromolecular interaction partners in achieving stable PTEN2 three-dimensional structure (Extended Data Fig. 6c). Notably, the PTEN2b variants lacking complete (PTEN2b ${ }^{\Delta n \text { ter }}$ ) or partial N-terminus $\left(P T E N 2 b^{\Delta 1-131}\right)$ could not properly localize to TGN (Fig. 6d and Extended Data Fig. 6d-f), where in addition to the cytosol PTEN2s normally accumulate ${ }^{15}$ (Extended Data Fig 4c). PTEN1 isoform, shown to be restricted to pollen grains ${ }^{37}$, when expressed from the PTEN2b promoter cannot be detected in PTEN2b expressing tissues. Here it could be speculated that the long PTEN2 N-terminus (not present in PTEN1) in important not only for TGN localization but also for protein stabilization (Fig. 6d). Hence, it appears possible that PTEN2b Nter-mediated TGN anchoring is critical for PTEN2 functionality in vacuolar remodeling and contribute to the evolution of land plants 


\section{DISCUSSION}

Eukaryotic cell compartmentalization occurred evolutionary concomitant with cell enlargement as the plasma membrane surface was not sufficient to provide all membrane-dependent functions ${ }^{38}$. There are different hypotheses explaining the origin of different membrane-bound organelles such as the endosymbiotic origin of mitochondria and chloroplasts, de novo formation of peroxisomes or transformation of existing endomembrane structures into new ones ${ }^{39-41}$. PSV formation represents an example when an existing compartment is remodeled into an organelle with a different function $^{22}$. Yet, very little is known about the mechanisms underpinning this functional reprogramming process. Here we showed a PTEN2-mediated mechanisms by which numerous PSVs are formed by the fragmentation of EVs following a cleavage furrowresembling mechanism (Fig. 1). Without PTEN2 enzymes the EV becomes hyper compartmentalized instead of fragmented. Interestingly, the observed EV membrane invaginations in pten $2 a$ pten $2 b$ mutants resemble formation of the mitochondrial cristae or chloroplast thylakoid membranes especially when the membrane is seen as pinched off (Fig. 1b) $38,41,42$.

As a typical cleavage furrow requires cytoskeleton involvement, it is tempting to speculate the importance of the cytoskeleton during vacuole division. Although actin was reported to be important in lytic vacuole tubularization, it is not clear if actin prevents vacuole expansion by physical constriction or by preventing actin-dependent membrane delivery to the vacuole ${ }^{43}$. Notably, vacuole invaginations can occur in a cytoskeleton independent fashion as reported during microautophagocytosis in yeast ${ }^{44}$. Moreover, the dynamics of contractile vacuoles present in protists depends rather on membrane tethering complexes than on the activity of cytoskeletal elements ${ }^{45,46}$. Consistent with the coupled occurrence of fusion and fission events in membrane homeostasis, our work revealed a PTEN2-mediated effect on vesicle fusion in xylem cells (Fig. 4). Inducible overexpression of PTEN2s potentiated tubular vacuole structures by preventing small vacuoles to fuse with it (arrows in Fig. 3b). Vacuole tubularization (as the most extreme form of membrane bending) is actually a core phenomenon necessary to form a cleavage furrow-like structure during EV division (Extended Data Fig. 4i). In pten2a pten $2 b$ mutants, vacuolar fission is hindered by the failure to form a symmetric ring of tonoplast invaginations at the division site (Fig. 1b). Coincidentally or not, plant PTEN2s appeared exactly in the 
Phragmoplastophyta clade and diverged their function from ancestral PTENs with the loss of cleavage furrow as cytokinesis mechanism ${ }^{47}$.

PTEN2s overexpressing lines provided a critical genetic tool to research the cell type specificities of vesicle trafficking in a developmental context. In this study we focused on the cell trafficking during xylem cell differentiation into a water conducting unit. We showed that PTEN2box prevents SCW formation in xylem cells, partially by inhibiting LAC17 secretion to the apoplast. As mutations in hemicellulose biosynthetic genes translate into xylem phenotypes, a potential suppressed delivery of hemicellulose to the apoplast may explain the lack of SCW cellulose in xylem cells upon PTEN2 $b_{o x}$ induction ${ }^{48-50}$. Furthermore, our current knowledge about xylem formation indicates that concomitant with SCW formation, hydrolytic enzymes necessary for PCD execution are loaded into the vacuole. Vacuoles store these enzymes in an inactive form until SCW formation is completed, ensuring the correct timing of PCD execution ${ }^{51}$. Our observations indicated that vacuolar acidification occurs just prior to PCD execution (Fig. 4k), suggesting a mechanism for the activation of hydrolytic enzymes. Subsequently, the vacuole swells, the tonoplast's permeability changes and finally the vacuole collapses releasing its content into the cytoplasm ${ }^{52,53}$. This process is thought to trigger a rapid cytoplasmic content degradation ${ }^{54}$. However, we showed that PCD-associated genes are correctly expressed and that protoxylem cells undergo cell death in the absence of SCW formation and without the formation of a large central vacuole, contrary to the expected sequence of xylem differentiation events ${ }^{55}$. Remarkably, previous studies reported autophagy as responsible for the gradual cellular content hydrolysis and reduced cytoplasmic density observed during the SCW biosynthesis ${ }^{53,56}$. Thus, autophagy may be an alternative mechanism for xylem cell clearance when vacuole-mediated pathway is inhibited. Indeed, we observed the creation of multiple cup-shaped vesicular structures resembling phagophore upon high PTEN2a induction (Fig. 4d) as well as that RabG3f positive small vacuoles creating grape-like aggregates (Extended Data Fig. 4h). It has been reported that another member of the same RabG3 subfamily, RabG3b can either stimulate or inhibit both autophagy and xylem formation, depending on its activation status ${ }^{57}$. Interestingly, the autophagy resembling pathway occurs also when massive amounts of synthesized proteins have to be delivered to the vacuole, as it occurs for seed storage proteins ${ }^{58,59}$. Similarly, some proteases necessary to mobilize the PSV content during germination was shown to also skip Golgi/TGN and directly from ER translocate 
to the vacuole 60,61 . Utilization of this direct ER-to-vacuole pathway may explain the absence of germination defects in pten2a pten $2 b$ seedlings incapable to form conventional PSVs. This notion is supported by our result that the direct ER-to-vacuole trafficking route remains unaffected in PTEN2box, evident by the presence of VHP1 and VHA-a3 in xylem vacuolar membranes (Fig. 3b and $4 b)^{18}$. Further investigation is needed to elucidate the exact downstream players in PTEN2s signaling cascade, and distinguish the biological importance of its dual phosphatase activity ${ }^{10}$, especially in the new light of its evolutionarily novel $\mathrm{N}$-terminal. However, it is tempting to speculate that the PTEN2 gene family diverged in green lineage to control vacuolar morphology and dynamics as their emergence coincided with vacuole enlargement during evolution. 


\section{METHODS}

\section{Plant materials and growth conditions}

Arabidopsis thaliana ecotype Columbia-O (Col-O) was used as wild-type control in all cases. Seeds of pten2a (SALK_114721), pten2b (SALK_120020) and cesa6 (SALK_004587) were obtained from the Nottingham Arabidopsis Stock Centre and combined by crossing. Homozygous lines were selected by genotyping using the primers listed in Supplementary Table 2. The following transgenic lines used in this study were described elsewhere: MYB46::GFP62, DMP4::H2A-GFP63, EXI1::H2AGFP ${ }^{63}, \quad$ PASPA3::H2A-GFP63, RNS3::H2A-GFP63, SCPL48::H2A-GFP, PASPA3::TolM ${ }^{28}$, VHA-a3::VHA-a3-GFP'18, VHP1::VHP1-GFP64, VHA-a1::VHA-a1RFP65, CESA6::YFP-CESA6 ${ }^{66}, \quad$ LAC17::LAC17-mCHERRY ${ }^{33}, \quad$ BRI1::BRI1mCITRINE ${ }^{67}$, UBQ::Rha1-YFP (W7Y) ${ }^{68}$, UBQ::RabG3f-YFP $(W 5 Y)^{68}$. These lines were combined with mutants or other transgenics by crossing. Following constructs (detailed information in Supplementary Table 3) were generated in this study: TIP3;2::TIP3;2-GFP, UBQ::XVE::PTEN1, UBQ::XVE::PTEN2a, UBQ::XVE::PTEN2b, VND7::NLS-3xVENUS, CESA7::NLS-3xVENUS, CESA4::NLS-3xVENUS, CESA8::NLS-3XVENUS, XCP1::XCP1-mCHERRY, BFN1::NLS-dtTOMATO, PTEN2b::PTEN2b-CITRINE, PTEN2b::PTEN2b-mCHERRY, UBQ::XVE::CrPTEN, UBQ::XVE::MPPTEN2, UBQ::XVE::PTEN2b $b^{\triangle C \text { ter }, ~ U B Q:: X V E:: P T E N 2 b ~} b^{\triangle N \text { Ner }}$, UBQ::XVE::PTEN2b $b^{41-131}, \quad$ UBQ::XVE::N1-PTEN2b, PTEN2b:: PTEN2b ${ }^{\triangle N \text { ter }} \quad$ CITRINE, PTEN2b:: PTEN2b $b^{41-131}$-CITRINE, PTEN2b::PTEN1-CITRINE. Constructs were transformed into Col-0, pten2a pten $2 b$ and marker lines (unless indicated) using Agrobacterium-mediated floral dip transformation according to standard procedures. For in vitro growth, seeds were surfaced sterilized, stratified 2 days at $4^{\circ} \mathrm{C}$ and grown on $0.5 \times$ Murashige and Skoog (MS, Duchefa) medium with MES buffer, pH 5.7, 0.7\% agar, and $1 \%$ sucrose. Seedlings were grown in vertical plates under continuous light conditions. The estradiol inducible lines were either germinated or transferred to identical media (48h treatments) containing $0.2 \mu \mathrm{M}$ or $2 \mu \mathrm{M}$ estradiol (ES, Sigma Aldrich). Seedlings were analyzed six days after germination unless specified otherwise. 


\section{Cloning procedures}

To generate pPROMOTER::NLS-3xVENUS reporter lines, the genomic region upstream the ATG of VND7 (1596bp), CESA7 (1153bp), CESA4 (1939bp), CESA8 (1949bp), or BFN1 (1975bp) was PCR-amplified using the primers listed in Supplementary Table 2. The resulting fragments were cloned into $p D O N R P r-P 1 r$ (Gateway) and subsequently recombined together with a $p E N z e o-N L S-3 x V E N U S^{69}$ plasmid into EDO097pFR7m24G70. Entry clone with BFN1 promoter was recombined together with $p E N-L 1-N L S-t d T O M A T O-L 2$ (Gateway) into destination vector pK7m24GW2 (Gateway) plasmid following the manufacturer instructions (Gateway, Invitrogen).

TIP3;2::TIP3;2-GFP line was generated by synthetizing TIP3;2 codding genomic sequence together with $845 \mathrm{bp}$ of promoter region upstream of ATG (as found in The Arabidopsis Information Resource Platform) and cloned into pDONRP4-P1r. Obtained entry clone was recombined with $p E N-L 1-G F P-L 2$ into EDO097pFR7m24G ${ }^{70}$.

Protein overexpression was achieved by estradiol XVE system (Gateway plasmid pMDC $7^{71}$ ). Coding sequences of PTEN1, PTEN2a and PTEN2b were amplified from Arabidopsis genomic DNA. MpPTEN2 (Mapoly0016s0179) was PCR amplified from Marchantia cDNA. CrPTEN (Cre06.g308400) was in vitro synthesized (Invitrogen). DNA amplicons containing attB1-B2 sites were and recombined into pMDC7 via pEN207 (PTEN1 and PTEN2b) or pEN221 (MpPTEN2 and CrPTEN) Gateway plasmids. PTEN2a was firstly cloned into $p 17 A C C D 2 P$, a plasmid created in this study. p17ACCD2P contains multicloning restriction sites: 5'- GAA TTC GAA GCT CGG TAC CCG GGG ATC CTC TAG AGT CGA CCT GCA GGC CCA TGG TGA CTA GTC AAG CTT - 3' between attL1 and attL2 Gateway recombination sites, thus providing direct creation of an entry clone without BP reaction.

Translational reporters were created by amplifying promoter regions of: PTEN2a (1116bp), PTEN2b (1173bp) and XCP1 (1601bp) and cloned into pDONRPr-P1r. Codding regions were amplified from whole seedling cDNA (for PTEN2a, PTEN2b and XCP1) or pollen enriched cDNA (PTEN1). Entry clones were made using pDONR207 except for PTEN2a, were $p 17 A C C D 2 P$ was used. Final constructs were generated by recombining the entry clones into $p H 7 m 34 G W$. Similarly, the distinct $P T E N 2 b$ protein variants (PTEN2b $b^{\Delta N t e r}, P T E N 2 b^{\triangle C t e r}, P T E N 2 b^{\Delta 1-131}$ and N1-PTEN2b) were cloned in frame with CITRINE by LR recombination into $p H 7 m 34 G W$ or for overexpression into pMDC7. Primers using for cloning can be found in Supplementary Table 2. 


\section{Histological analysis}

PSV biogenesis was visualized in epidermal cells of Arabidopsis embryos extracted from green siliques (stages I-III), yellow siliques (stages IV-V), dry seeds after $4 \mathrm{~h}$ imbibition in water (stage VI) or 24-48h after imbibition in constant light conditions (stages VII-VIII). Cellulose (by Calcofluor White from Sigma-Aldrich) and lignin staining (Fuchsin from Sigma-Aldrich) were performed after seedlings clearing following ClearSee protocol as previously described ${ }^{72}$. DAPI (4',6-diamidino-2-phenylindole, Sigma-Aldrich) staining used to verify PCD status of xylem cells was performed after fuchsin staining when seedlings were exposed to $50 \mu \mathrm{g} / \mathrm{ml}$ DAPI in $1 \mathrm{x}$ PBS (phosphate-buffered saline) with $1 \%$ TRITON-X for $1 \mathrm{~h}$ with gentle shaking. After thorough washes in 1x PBS, seedlings were visualized with a confocal microscope. Live imaging of green fluorophores was performed upon propidium iodide (SigmaAldrich) or FM4-64 (Invitrogen) staining according to standard procedures ${ }^{65}$. Chemical treatment with Brefeldin A (BFA, Sigma-Aldrich) was performed for duration of 90 minutes in concentration of $50 \mu \mathrm{M}$ in liquid 0.5 MS media. Transverse plastic sections of roots were performed and visualized as previously described ${ }^{73}$.

\section{Confocal microscopy and image analysis}

Confocal laser-scanning microscopy images were obtained using either a Leica SP8 (in Fig. 4, Fig. 5 [b-g, k], Fig. 6d, Fig. 7c-d and Extended Data Fig. 5 [a, f-h]) or Zeiss LSM 780 microscopes. Blue dyes such as Calcofluor White and DAPI were excited at $405 \mathrm{~nm}$ and detected at $430-485 \mathrm{~nm}$ as well as PSV autofluorescence. Green fluorophores (GFP, CITRINE, Venus, YFP) were excited at 488nm and detected between 500-550nm. Red fluorophores and dyes (RFP, mCHERRY, tdTOMATO, propidium iodide, FM4-64 and fuchsin) were excited at 561nm and detected at 590650nm. 63x Oil Plan-Apochromat DIC M27 objective was used to visualize Arabidopsis embryos, otherwise the 40x water LD C-Apochromat M27 objective was used on Zeiss LSM 780 microscope. For two photon fluorescence excitation, Mai Tai XF (Spectra-Physics) laser at 980nm was used to excite GFP, YFP and CITRINE, while InSight DeepSee (Spectra-Physics) at 1060nm was used to excite RFP and mCHERRY fluorophores. Here, 40x water HC PL IRAPO objective was used. Signal detection was collected with external detectors based on FITC (525/50nm) / TexasRed (617/70nm) filters. Images were processed in ImageJ or Imaris image processing 
software. When image colors were inverted and/or adjusted, all images belonging to one experiment were processed simultaneously. Scale bars were added in ImageJ.

\section{Transmission electron microscopy}

The root of 7-day-old seedlings was mounted in 1-hexedecene (Sigma) on a carrier with a $2 \mathrm{~mm}$ diameter and high-pressure frozen using the Leica EM HPM100. Then the samples were substituted in $1 \% \mathrm{OsO}_{4}$ for $6 \mathrm{~h}$ at $-90^{\circ} \mathrm{C}$, followed by $3 \mathrm{~h}$ at $-60^{\circ} \mathrm{C}$, 3h at $-30^{\circ} \mathrm{C}$, and $1 \mathrm{~h}$ at $0^{\circ} \mathrm{C}$. After one hour incubation, samples were rinsed twice with anhydrous acetone and incubated for $2 \mathrm{~h}$ in 33\% Epon/Araldite (Epon 812-Sigma, Durcupam ACM-Sigma, Dibutylphtalat-Sigma) in anhydrous acetone at $4^{\circ} \mathrm{C}$. Next, samples were incubated in $66 \%$ Epon/Araldite in anhydrous acetone at $4^{\circ} \mathrm{C}$ overnight, and finally embedded in 100\% Epon/Araldite. Samples were then trimmed using a glass knife and 70nm sections were cut with a diamond knife (DiATOME) using a ultramicrotome (Leica Ultracut UCT). Sections were assembled on a grid $(2 \mathrm{~mm} \times 1 \mathrm{~mm}$ slit diaphragm, PLANO), coated with formvar (0.85\% formvar in 1,2-dichlorethane). Contrast of the samples on the grids were enhanced with lead citrate. Samples were examined using the FEI Tecnai G2 Spirit transmission electron microscope with two digital CCD cameras (Gatan Orius 1000, FEI Eagle).

\section{RNA-extraction, RT-qPCR and Western Blot analysis}

Total RNA was extracted from 7-day-old seedlings using RNeasy® Plant Mini-Kit (QIAGEN) and treated with RNase-Free DNase (QIAGEN). cDNA synthesis was performed using RevertAid First Strand cDNA synthesis kit (Thermoscientific). RTqPCR was performed using KAPA SYBR® FAST (KAPA BIOSYSTEMS), primers listed in Table $S 1$ and $2 \mu \mathrm{L}$ of 1:10 dilution of cDNA. All reactions were performed in triplicates. Expression data derived from $\mathrm{Cp}$ values calculated according to the second derivative maximum method (LightCycler®LC480 II, Roche) and normalized to the expression of PDF2 (At4g04890). To detect PTEN2-CITRINE protein in the transgenic lines, total proteins of 7-day-old seedlings were extracted using Laemmli buffer (v/v), separated in $12.5 \%(\mathrm{w} / \mathrm{v})$ sodium dodecyl sulfate-polyacrylamide gel electrophoresis (SDS-PAGE) and transferred to an AmershamTM HybondTM 0.45 $\mu$ m polyvinylidene difluoride (PVDF) membrane (Merck). CITRINE-fusion proteins were detected with anti-GFP antibody (JL-8, Takara Bio Clontech, dilution: 1:5000, overnight incubation) and anti-Mouse IgG (Fc-specific)-Peroxydase (Sigma, dilution: 1/10000, 1.5hr 
incubation). Anti-Hsp90 (Agrisera, dilution: 1:2500, overnight incubation) and antiRabbit IgG (whole molecule)-HRP (Sigma A0545, dilution: 1:5000, 1.5hr incubation) were used as loading control. Chemiluminescence was revealed using Rotiß-Lumin (ROTH) as substrate and imaged with a ChemiDoc Touch Imaging System (Bio-Rad).

\section{Phylogenetic analysis}

Protein sequences of PTEN2b homologous from 142 plant species from Phytozome, National Center from Biotechnology Information and Plaza 4.0 databases were analyzed using the following criteria: $\mathrm{E}$ value cut off $10^{-10}$ (Phytozome), total score cut off 100 (NCBI) and score cut off 100 (Plaza). Next, we removed manually the sequences whose identity was higher than $99 \%$ within the same species as well as the incomplete sequences. We only represented one splice variant for each locus and remove miss-aligned sequences. The resulting sequences were aligned using CLUSTAL OMEGA algorithm and the tree was generated by using FigTree version 1.4.3 software (http://tree.bio.edu.ac.uk/software/figtree/) and color-coded edited manually.

\section{Bioinformatic analysis of physicochemical protein properties}

The coding sequences of the N-terminal domain of CrPTEN, AtPTEN1, AtPTEN2a, AtPTEN2b and MpPTEN2 were aligned (CLUSTAL OMEGA) and the presence of membrane binding domains was predicted using a $\mathrm{BH}$ score above 0.6 as described by Brzeska et $\mathrm{al}^{74}$. By using IUPred2A score ${ }^{75,76}$, domains with values above 0.5 were assigned as highly probably intrinsic disorder domains.

\section{ACKNOWLEDGEMENTS}

The authors thank Dr. K. Schumacher, Dr. Y. Oda and Dr. S. Fujita for kindly providing transgenic material, Dr. J. Westermann for providing us with Marchantia cDNA, Dr. A. Ruiz-Sola, Dr. J Alassimone and K. Kirchoff for great technical assistance. We thank J. Kusch and T. Scwartz from ScopeM for their support in handling the confocal microscopes and image processing and to University of Zürich TEM services for technical assistance. B.G. was financially supported by Vontobel, A.H. and T.M.D.C. by ETH-Foundation grants, and C.C., N.B-T., and E.R. by ETH core funding. This work was also funded by the Swiss National Foundation (SNF_31003A_160201 to A.R.-V.). 


\section{FIGURE LEGENDS}

Figure 1: Embryonic vacuole division involves cleavage furrow formation and requires PTEN2 activity. a-h, Representative confocal images of vacuoles in epidermal cells in Arabidopsis embryos extracted from green siliques (stages I-III), yellow siliques (stages IV-V), dry seeds (VI), germinated for $24 \mathrm{~h}$ (stage VII) or $48 \mathrm{~h}$ (stage VIII). Tonoplast (vacuolar membrane) decorated by TIP3;2-GFP (in green). a, Comparative seven stages of TIP3;2 dynamics between wild type and pten2a pten $2 b$. At the onset of its expression, TIP3;2 accumulates in endoplasmic reticulum close to plasma membrane (orange arrows), or follow nuclear shape (yellow arrows). First tonoplast is visible in stage II marked with a white arrow. Small pre-embryonic vacuoles fuse in stage III (fusion sites are marked with blue arrows). Please note that image of EVs in pten2a pten $2 b$ in stage IV is slightly advanced than in wild type. In stages V-VII PSV exhibit autofluorescence in blue part of the spectrum (represented blue in images). Magenta shows autofluorescence in red part of the spectrum. b, EV division starts by tonoplast invagination at division site visible as a circle (white dashed arrows). In addition to the lack of synchronicity in tonoplast invaginations, in pten2a pten $2 b$ double mutants, membrane unilaterally ingress (red dashed arrow) and can reach the other side of the vacuole, however the division does not occur. Possible cause is the absence of membrane bending into hourglass shape as visible in WT (yellow asterisk). The ingrown membrane possibly rolls into a cylindrical shape as visible in $3 \mathrm{D}$ reconstructions of the late-stage $\mathrm{V}$ in double mutants. $\mathbf{c}$, Representative confocal images of storage vacuoles' autofluorescence in epidermal cells of Arabidopsis embryos extracted from dry seeds and germinating seedlings in wild type and pten $2 a$ pten $2 b$ mutants. Scale bars represent $20 \mu \mathrm{m}$. d, Germination $24 \mathrm{~h}$ after imbibition in indicated genotypes. Error bars represent SE. n>500. e-f, Quantification of root length (e) and meristem size (f) in 6 days old seedlings of the indicated genotypes. $n>30$ (e) or $n=10$ (f). All error bars represent standard error. n.s., not significant; $* * \mathrm{p}<0.01$.

Figure 2: PTEN2s prevent xylem cell differentiation. a, Representative confocal images of protoxylem cells in the indicated genotypes stained with Calcofluor White (cellulose in cyan) and Fuchsin (lignin in magenta). Secondary cell wall (SCW) was also visualised by transmission light (TM). Scale bars represent $20 \mu \mathrm{m}$. b, 
Quantification of xylem phenotypes observed in the roots displayed in a. The two phenotypes observed and scored were the total absence of xylem strands (without xylem) or the appearance of several protoxylem cells with SCW (islands). c, Representative images of indicated xylem differentiation markers in wildtype (WT) and seedlings incubated in $0.2 \mu \mathrm{M}$ estradiol for $48 \mathrm{~h}$ to trigger PTEN2 $b_{\text {ox. }}$. Roots were stained with Calcofluor White (cyan) and fuchsin (magenta). Marker lines: VND7 (VASCULAR RELATED NAC-DOMAIN PROTEIN 7), MYB46 (MYB DOMAIN PROTEIN 46), CESA7 (CELLULOSE SYNTHASE CATALYTIC SUBUNIT 7), CESA4 (CELLULOSE SYNTHASE A4), CESA8 (CELLULOSE SYNTHASE 8), DMP4 (DUF679 DOMAIN MEMBRANE PROTEIN 4), EXI1 (EXITUS 1). Scale bars represent $20 \mu \mathrm{m}$. d, Transmission electron microscopy images of differentiating proto- (px) and metaxylem ( $\mathrm{mx}$ ) cells in WT and PTEN2box. Xylem cells in WT formed thick secondary cell wall (SCW), vacuoles are enlarging in $\mathrm{mx}$ while $\mathrm{px}$ underwent programmed cell death. PTEN2b overexpression prevents SCW formation while $\mathrm{mx}$ cells contain multiple small vacuoles. Here px cell also underwent clearance.

Figure 3: Regulation of xylem vacuolar biogenesis regulation by PTEN2s. a, 3D reconstruction of VHP1-GFP decorated vacuolar compartments in protoxylem cells in wild type plants at progressive developmental stages counterstained with propidium iodide $(\mathrm{PI})$ to label cell wall. Yellow arrows mark small vacuole-like compartments and black arrows mark tubular connecting membranes. For easier visualization, protoxylem cells margins were squared by a white dashed line. Scale bars represent $20 \mu \mathrm{m}$. b, Representative images of mature protoxylem cells in the indicated genotypes, visualized as in a. Scale bars represent $20 \mu \mathrm{m}$. Black arrows mark tubular connecting membranes. c, Comparison of vacuolar morphology in mature epidermis (ep), cortex (co), endodermis (en), pericycle (pc) and protoxylem (asterisk) between indicated genotypes. Scale bars represent $20 \mu \mathrm{m}$.

Figure 4: PTEN2s inhibit vacuolar and secretion trafficking pathway in xylem cells but not PCD. a, Schematic representation of analysed trafficking pathways important for xylem cell differentiation. b-g, Representative images of the corresponding xylem cells in wild type (WT) and seedlings with $0.2 \mu \mathrm{M}$ estradiolmediated PTEN2b induction for $48 \mathrm{~h}$ visualizing different trafficking markers: tonoplast 
marker VHA-a3 (VACUOLAR PROTON ATPASE A3) (b), xylem specific promoter PASPA3 (PUTATIVE ASPARTIC PROTEINASE A3) driving expression of TolM (tonoplast integrity marker) showing GFP in ER and a vacuolar targeted mRFP (c), vacuolar cargos CESA6 (CELLULOSE SYNTHASE SUBUNIT A6) (d) and XCP1 (XYLEM CYSTEINE PEPTIDASE 1) (e), secreted cargo LAC17 (LACCASE 17) (f). g, non-secreted LAC17 is not delivered into VHP1-labeled vacuoles. $\mathbf{h}-\mathbf{j}$, PCD execution occurs even without SCW formation in PTEN2box. $\mathbf{h}$, DAPI-stained nuclei are absent in the cells where the xylem-specific expression of MYB46 ceased due to the PCD execution (white arrows). Scale bars represent $20 \mu \mathrm{m}$. i, Toluidine-stained root crosssections of the indicated genotypes. Xylem secondary cell wall stains bright blue as visible in WT but absent in PTEN2box overexpression where xylem cells appear collapsed as in transmission electron microscopy images (j). j, Transmission electron microscopy images of the indicated genotypes. Notice a high number of small vacuoles and aggregates in PTEN2box. Yellow dashed circle highlights a cup-shaped phagophore. Scale bars represent $2 \mu \mathrm{m}$. k, Xylem vacuole acidification in wild type prior PCD. Note faster fading of $\mathrm{pH}$-sensitive YFP in comparison to $\mathrm{pH}$-tolerant mCHERRY. The cell where acidification occurs is encircled with a white dashed line. Scale bars represent $10 \mu \mathrm{m}$. I, continuation cell from $\mathrm{k}$, where PCD is executed and mCHERRY signal disappears too. Scale bars represent $10 \mu \mathrm{m}$

\section{Figure 5: PTEN2s functions in vacuolar fusion and xylem differentiation were} conserved through evolution. a, Schematic tree of the evolution of plant PTEN subfamilies. b, Phylogenetic tree of 418 plant PTENs from 142 plant species. For simplification, only the isoforms of species of interest have been represented. Details about all the sequences and the complete distribution of the isoforms in the three subfamilies (ancestor-like PTENs, PTEN1s, PTEN2s) can be found in Supplementary Table 2. c, Representative confocal microscopy images of fuchsin-stained protoxylem strands from roots grown on mock conditions or upon $2 \mu \mathrm{M}$ estradiol-mediated induction for 48h of Chlamydomonas reinharditi PTEN (CrPTEN), Arabidopsis thaliana PTEN1 (AtPTEN1), PTEN2a (AtPTEN2a), and PTEN2b (AtPTEN2b) and Marchantia polymorpha PTEN2 (MpPTEN2). d, Representative confocal microscopy images of vacuolar morphology in mature xylem cells (VHP1-GFP labels tonoplast, propidium iodide stains cell wall) of inducible over-expressor lines of the different PTEN isoforms 
mentioned above. PTEN over-expression was induced by $2 \mu \mathrm{M}$ estradiol for $48 \mathrm{~h}$. Protoxylem gaps are highlighted with white dashed lines. Scale bars represent $20 \mu \mathrm{m}$.

Figure 6: A conserved domain within PTEN2s $\mathbf{N}$-terminal sequence is critical for their functionality and TGN anchoring. a, Schematic representation of the PTEN enzymes from Homo Sapiens (Hs), Arabidopsis (At), Marchantia (Mp) and Chlamydomonas $(\mathrm{Cr})$ analysed in this study. On the right are represented the truncated version of PTEN2b without the entire C- (PTEN2 $b^{\Delta C \text { ter }}$ ) or N-terminal (PTEN2b ${ }^{\triangle N \text { Nter }}$ ) sequences, PTEN2b with a partial N-terminal sequences $\left(P T E N 2 b^{\Delta 1-}\right.$ ${ }^{131}$ ) and the hybrid version with PTEN1 N-terminal (N1-PTEN2b). Colour filled boxes represent phosphatase catalytic domains whereas empty squared boxes represent C2 domains. b, Representative confocal microscopy images of fuchsin-stained protoxylem strands after $2 \mu \mathrm{M}$ estradiol-mediated induction for $48 \mathrm{~h}$ of indicated PTEN2b versions. Protoxylem gap cells are highlighted with white dashed lines. c, Representative images of vacuole morphology in mature xylem cell upon 48h overexpression of indicated PTEN2b variants. VHP1-GFP labels tonoplast, while propidium iodide labels cell wall. Protoxylem gap cells are highlighted with white dashed lines. d, Representative confocal images of 6-day-old plants harbouring indicated constructs illustrating the dependence of PTEN2b localization at TGN to its $\mathrm{N}$-terminal. Scale bars represent $20 \mu \mathrm{m}$.

\section{Extended Data Figure 1: Aberrant EV division does not affect post-embryonic} development. a-c, Representative confocal images of aquaporin TIP3;2-GFP (green) distribution during embryogenesis. a, Similar to TIP3;2, VHP1 tonoplast marker also labels ER. Magenta shows autofluorescence from chloroplasts visible in embryos isolated from green siliques. b, 3D maximal projection of a stage III vacuole corresponding to 2D image shown in Figure 1a. c, Small vacuoles labelled with TIP3;2 close to EV tonoplast preceding the cleavage furrow division. Magenta shows autofluorescence detected in red part of the spectrum. Scale bars represent $20 \mu \mathrm{m}$. $\mathbf{d}$, Germination rate between indicated genotypes. Error bars represent SE. $n>500$. e, Analysis of normalized, relative PTEN2b overexpression by qRT-PCR in two independent transgenic lines induced with $2 \mu \mathrm{M}$ estradiol for the duration of 6 days. Error bars represent SE among three independent biological replicates. f, 
Representative confocal images of embryos dissected from dry seeds, $24 \mathrm{~h}$ and $48 \mathrm{~h}$ after imbibition of the indicated genotypes, stained with Calcofluor White (cellulose in cyan) and fuchsin (lignin in magenta). Yellow arrows mark xylem discontinuities in seedlings with induced PTEN2b overexpression from imbibition $(2 \mu \mathrm{M}$ estradiol). Note the appearance of differentiated xylem cells (magenta) only $48 \mathrm{~h}$ after germination. Scale bars $200 \mu \mathrm{m}$. g, Root length quantification of 6-days-old seedlings illustrate dose dependant effect of PTEN2b overexpression after $48 \mathrm{~h}$ of estradiol induction. Error bars represent SE. $\mathrm{n}>40 \mathrm{~h}$, PTEN2b overexpression prevents xylem differentiation in T3.4.40 line in both proximal and distal part of the root. Higher overexpression in the line T3.13.41 dramatically shortens the root length (g) but does not prevent xylem differentiation in younger distal root parts. Undifferentiated xylem is only labelled with MYB46 marker in yellow, while differentiated xylem is labelled with fuchsin staining for lignin in magenta, or white (overlap between yellow and magenta). Asterisk labels xylem position within vascular cylinder. White arrows label ectopic lignification in endodermis. Scale bars represent $100 \mu$ m. n.s., not significant; $* p<0.05 ; * * p<0.01$; $* * * \mathrm{p}<0.001$.

\section{Extended Data Figure 2: PTEN2b overexpression does not alter the expression} of genes associated with xylem PCD execution. a, Representative confocal microscopy images of the mature protoxylem cells stained with Calcofluor White for cellulose (cyan) and fuchsin for lignin (magenta). PTEN2b was induced for $48 \mathrm{~h}$ with $0.2 \mu \mathrm{M}$ estradiol. Note the expression of genes associated with PCD such as the PUTATIVE ASPARTIC PROTEINASE A3 (PASPA3), RIBONUCLEASE 3 (RNS3), SERINE CARBOXYPEPTIDASE-LIKE 48 (SCPL48) and BIFUNCTIONAL NUCLEASE 1 (BFN1) can still be detected in seedlings with PTEN2b upregulation. Asterisks mark protoxylem strands. Scale bars represent $20 \mu \mathrm{m}$.

\section{Extended Data Figure 4: PTEN2b colocalize to TGN and impinges on vacuolar} and cell secretion pathways. a, Brassinosteroid receptor BRI1 (BRASSINOSTEROID INSENSITIVE 1) cannot be delivered to xylem vacuoles upon PTEN2b overexpression. Seedlings were counterstained with propidium iodide $(\mathrm{PI})$. b, PTEN2b colocalize with cellular compartments early labelled with FM4-64 (magenta). c, PTEN2b partially colocalize with PROTON ATPASE A1 (VHA-a1) in 
TGN (arrows). d, PTEN2b partially colocalize with CELLULOSE SYNTHASE SUBUNIT A6 (CESA6). e, cesa6 mutant cannot rescue secondary cellulose building upon PTEN2b overexpression. f, Aggregates of vesicles carrying vacuolar destined cargo (CESA6 in green) and secretion cargo (LAC17 in red) do not colocalize. Arrows' color corresponds to fluorophores and points the aggregates where proteins do not colocalize g, Multivesicular body (MVB) marker Rha1 (ARABIDOPSIS RAB HOMOLOG F2A) creates aggregates in xylem cells upon PTEN2b upregulation. $\mathbf{h}$, Prevacuolar compartment and tonoplast marker RabG3f (RAB GTPASE HOMOLOG G3F) upon prolonged PTEN2b overexpression creates grape like structures in vicinity of the central vacuole in mature epidermal cells. Asterisk labels xylem strands. Scale bars represent $20 \mu \mathrm{m}$. i, Schematic representation of membrane phenomena regulated by PTEN2s.

\section{Extended Data Figure 6: The N-terminal domains of PTEN1 and PTEN2s exhibit different biochemical properties that determines their subcellular localization.}

a, Alignment of N-terminal PTEN sequences from: human (HSPTEN), Chlamydomonas (CrPTEN), Arabidopsis (AtPTEN1, AtPTEN2a and AtPTEN2b), and Marchantia (MpPTEN2) proteome assemblies obtained using CLUSTAL OMEGA. Identical amino acids are represented in green while similar amino acids are represented in magenta. b, Prediction of membrane binding domain in PTEN Nterminal sequences of indicated isoform using $\mathrm{BH}$ score ${ }^{74}$. Domains with values above 0.6 are predicted to be membrane binding domain. $\mathbf{c}$, Prediction of intrinsic disordered region in $\mathrm{N}$-terminal sequences of indicated isoform using IUPred2A score ${ }^{75,76}$. Regions with values above 0.5 are supposed to be enriched in intrinsic disorders. The grey areas highlight the domain identified in PTEN2b (amino acid 132-188) as responsible for its functionality. $\mathbf{d}$, Representative confocal images of 6 day-old plants expressing the TGN marker VHAa1-RFP together with PTEN2b::PTEN2b-citrine or PTEN2b::PTEN2b $b^{\Delta 1-131}-C I T R I N E$. Arrows indicate the position of some of the dotted structures observed for PTEN2b::PTEN2b41-131-CITRINE. e, Representative confocal images of 6 day-old plants expressing PTEN2b::PTEN2b $b^{\triangle 1-131}-C I T R I N E$ with PTEN2b::PTEN2b-mCHERRY. Please note the co-localization of both constructs. $\mathbf{f}$, Confocal images of 6 day-old plants expressing PTEN2b::PTEN2b-1-131-citrine and treated with DMSO or $50 \mu \mathrm{M}$ BFA for $1.5 \mathrm{~h}$. Scale bars represent $10 \mu \mathrm{m}$. 


\section{Supplementary Figure 1: Transgenic lines validation (Supporting data for}

Figures 6 and 7). a, qPCR analyses confirmed the over-expression of PTEN in different inducible lines described in Fig. 6 and Fig. 7. RNA was extracted from roots of 7 day-old plants treated with DMSO or $2 \mu \mathrm{M}$ estradiol for $48 \mathrm{hrs}$. Expression values of the different genes of interest in estradiol-treated plants were normalized by the corresponding expression values measured in DMSO-treated plants. Values represent the mean of two biological replicates (both including three technical replicates), error bars indicate the standard deviation. b, qPCR analyses revealed the presence of CITRINE mRNA in independent PTEN2b::PTEN1-CITRINE that do not exhibit any fluorescence signal in root cells (Fig 6). Values represent the mean of three technical replicates, error bars indicate the standard deviation. $\mathbf{c}$, The expression of the different Nter-truncated versions of PTEN2b tagged with citrine (Fig 6) was confirmed by Western blot using anti-GFP antibody. Anti-Hsp90 was used as a loading control. The star indicates the presence of an unspecific band.

Supplementary Table 1: Protein sequences used to build the phylogenetic tree.

Supplementary Table 2: Primers used in this study.

Supplementary Table 3: Constructs generated in this study.

\section{REFERENCES}

1 Peters, C., Baars, T. L., Buhler, S. \& Mayer, A. Mutual control of membrane fission and fusion proteins. Cell 119, 667-678, doi:10.1016/j.cell.2004.11.023 (2004).

2 Martens, S. \& McMahon, H. T. Mechanisms of membrane fusion: disparate players and common principles. Nat Rev Mol Cell Biol 9, 543-556, doi:10.1038/nrm2417 (2008).

3 Lenz, M., Morlot, S. \& Roux, A. Mechanical requirements for membrane fission: common facts from various examples. FEBS Lett 583, 3839-3846, doi:10.1016/j.febslet.2009.11.012 (2009).

4 Schmid, S. L. \& Frolov, V. A. Dynamin: functional design of a membrane fission catalyst. Annu Rev Cell Dev Biol 27, 79-105, doi:10.1146/annurev-cellbio-100109-104016 (2011).

5 lijima, M., Huang, Y. E., Luo, H. R., Vazquez, F. \& Devreotes, P. N. Novel mechanism of PTEN regulation by its phosphatidylinositol 4,5-bisphosphate binding motif is critical for chemotaxis. J Biol Chem 279, 16606-16613, doi:10.1074/jbc.M312098200 (2004).

6 Derman, M. P. et al. The lipid products of phosphoinositide 3-kinase increase cell motility through protein kinase C. J Biol Chem 272, 6465-6470, doi:10.1074/jbc.272.10.6465 (1997).

7 Funamoto, S., Meili, R., Lee, S., Parry, L. \& Firtel, R. A. Spatial and temporal regulation of 3-phosphoinositides by PI 3-kinase and PTEN mediates chemotaxis. Cell 109, 611-623, doi:10.1016/s0092-8674(02)00755-9 (2002). 
8 Munnik, T. \& Testerink, C. Plant phospholipid signaling: "in a nutshell". J Lipid Res $\mathbf{5 0}$ Suppl, S260-265, doi:10.1194/jlr.R800098-JLR200 (2009).

9 Vermeer, J. E. et al. Visualization of Ptdlns3P dynamics in living plant cells. Plant J 47, 687-700, doi:10.1111/j.1365-313X.2006.02830.x (2006).

10 Pribat, A. et al. A novel class of PTEN protein in Arabidopsis displays unusual phosphoinositide phosphatase activity and efficiently binds phosphatidic acid. Biochem $\mathrm{J}$ 441, 161-171, doi:10.1042/BJ20110776 (2012).

11 Jurgens, G. Plant cytokinesis: fission by fusion. Trends Cell Biol 15, 277-283, doi:10.1016/j.tcb.2005.03.005 (2005).

12 Buschmann, H. \& Zachgo, S. The Evolution of Cell Division: From Streptophyte Algae to Land Plants. Trends Plant Sci 21, 872-883, doi:10.1016/j.tplants.2016.07.004 (2016).

13 Becker, B. Function and evolution of the vacuolar compartment in green algae and land plants (Viridiplantae). Int Rev Cytol 264, 1-24, doi:10.1016/S0074-7696(07)64001-7 (2007).

14 Beauzamy, L., Derr, J. \& Boudaoud, A. Quantifying Hydrostatic Pressure in Plant Cells by Using Indentation with an Atomic Force Microscope. Biophys J 108, 2448-2456, doi:10.1016/j.bpj.2015.03.035 (2015).

15 Delgadillo, M. O. et al. MTV proteins unveil ER- and microtubule-associated compartments in the plant vacuolar trafficking pathway. Proc Natl Acad Sci U S A 117, 9884-9895, doi:10.1073/pnas.1919820117 (2020).

16 Cui, Y. et al. A whole-cell electron tomography model of vacuole biogenesis in Arabidopsis root cells. Nature plants 5, 95-105, doi:10.1038/s41477-018-0328-1 (2019).

17 Kruger, F. \& Schumacher, K. Pumping up the volume - vacuole biogenesis in Arabidopsis thaliana. Semin Cell Dev Biol 80, 106-112, doi:10.1016/j.semcdb.2017.07.008 (2018).

18 Viotti, C. et al. The endoplasmic reticulum is the main membrane source for biogenesis of the lytic vacuole in Arabidopsis. Plant Cell 25, 3434-3449, doi:10.1105/tpc.113.114827 (2013).

19 Tanaka, Y. et al. Intra-vacuolar reserves of membranes during stomatal closure: the possible role of guard cell vacuoles estimated by 3-D reconstruction. Plant Cell Physiol 48, 1159-1169, doi:10.1093/pcp/pcm085 (2007).

20 Beilby, M. J. Chara braunii genome: a new resource for plant electrophysiology. Biophys Rev 11, 235-239, doi:10.1007/s12551-019-00512-7 (2019).

21 Cui, Y., Zhao, Q., Hu, S. \& Jiang, L. Vacuole Biogenesis in Plants: How Many Vacuoles, How Many Models? Trends Plant Sci 25, 538-548, doi:10.1016/j.tplants.2020.01.008 (2020).

22 Feeney, M., Kittelmann, M., Menassa, R., Hawes, C. \& Frigerio, L. Protein Storage Vacuoles Originate from Remodeled Preexisting Vacuoles in Arabidopsis thaliana. Plant Physiol 177, 241-254, doi:10.1104/pp.18.00010 (2018).

23 Gopaldass, N., Fauvet, B., Lashuel, H., Roux, A. \& Mayer, A. Membrane scission driven by the PROPPIN Atg18. EMBO J 36, 3274-3291, doi:10.15252/embj.201796859 (2017).

24 Tiedemann, J., Schlereth, A. \& Muntz, K. Differential tissue-specific expression of cysteine proteinases forms the basis for the fine-tuned mobilization of storage globulin during and after germination in legume seeds. Planta 212, 728-738, doi:10.1007/s004250000435 (2001).

25 Zheng, H. \& Staehelin, L. A. Protein storage vacuoles are transformed into lytic vacuoles in root meristematic cells of germinating seedlings by multiple, cell type-specific mechanisms. Plant Physiol 155, 2023-2035, doi:10.1104/pp.110.170159 (2011).

26 Gujas, B. et al. Perturbing phosphoinositide homeostasis oppositely affects vascular differentiation in Arabidopsis thaliana roots. Development 144, 3578-3589, doi:10.1242/dev.155788 (2017).

27 Lucas, W. J. et al. The plant vascular system: evolution, development and functions. $J$ Integr Plant Biol 55, 294-388, doi:10.1111/jipb.12041 (2013).

28 Fendrych, M. et al. Programmed cell death controlled by ANAC033/SOMBRERO determines root cap organ size in Arabidopsis. Curr Biol 24, 931-940, doi:10.1016/j.cub.2014.03.025 (2014). 
29 Watanabe, Y. et al. Cellulose synthase complexes display distinct dynamic behaviors during xylem transdifferentiation. Proc Natl Acad Sci U S A 115, E6366-E6374, doi:10.1073/pnas.1802113115 (2018).

30 Mottiar, Y., Vanholme, R., Boerjan, W., Ralph, J. \& Mansfield, S. D. Designer lignins: harnessing the plasticity of lignification. Curr Opin Biotechnol 37, 190-200, doi:10.1016/j.copbio.2015.10.009 (2016).

31 Takenaka, Y. et al. Patterned Deposition of Xylan and Lignin is Independent from that of the Secondary Wall Cellulose of Arabidopsis Xylem Vessels. Plant Cell 30, 2663-2676, doi:10.1105/tpc.18.00292 (2018).

32 Northcote, D. H., Davey, R. \& Lay, J. Use of antisera to localize callose, xylan and arabinogalactan in the cell-plate, primary and secondary walls of plant cells. Planta 178, 353-366, doi:10.1007/BF00391863 (1989).

33 Schuetz, M. et al. Laccases Direct Lignification in the Discrete Secondary Cell Wall Domains of Protoxylem. Plant Physiology 166, 798-U489, doi:10.1104/pp.114.245597 (2014).

34 Young, B., Wightman, R., Blanvillain, R., Purcel, S. B. \& Gallois, P. pH-sensitivity of YFP provides an intracellular indicator of programmed cell death. Plant Methods 6, 27, doi:10.1186/1746-4811-6-27 (2010).

35 Kleine-Vehn, J. et al. Differential degradation of PIN2 auxin efflux carrier by retromerdependent vacuolar targeting. Proc Natl Acad Sci U S A 105, 17812-17817, doi:10.1073/pnas.0808073105 0808073105 [pii] (2008).

$36 \mathrm{Han}, \mathrm{X}$. et al. Origin and Evolution of Core Components Responsible for Monitoring Light Environment Changes during Plant Terrestrialization. Mol Plant 12, 847-862, doi:10.1016/j.molp.2019.04.006 (2019).

37 Gupta, R., Ting, J. T., Sokolov, L. N., Johnson, S. A. \& Luan, S. A tumor suppressor homolog, AtPTEN1, is essential for pollen development in Arabidopsis. Plant Cell 14, 2495-2507 (2002).

38 Alberts, B. Molecular biology of the cell. 5th ed. edn, (Garland Science ; [London : Taylor \& Francis, distributor], 2008).

39 Joshi, A. S., Zhang, H. \& Prinz, W. A. Organelle biogenesis in the endoplasmic reticulum. Nat Cell Biol 19, 876-882, doi:10.1038/ncb3579 (2017).

40 Schrader, M. \& Pellegrini, L. The making of a mammalian peroxisome, version 2.0: mitochondria get into the mix. Cell death and differentiation 24, 1148-1152, doi:10.1038/cdd.2017.23 (2017).

41 Munoz-Gomez, S. A. et al. Ancient homology of the mitochondrial contact site and cristae organizing system points to an endosymbiotic origin of mitochondrial cristae. Curr Biol 25, 1489-1495, doi:10.1016/j.cub.2015.04.006 (2015).

42 Vothknecht, U. C. \& Westhoff, P. Biogenesis and origin of thylakoid membranes. Biochim Biophys Acta 1541, 91-101, doi:10.1016/s0167-4889(01)00153-7 (2001).

43 Scheuring, D. et al. Actin-dependent vacuolar occupancy of the cell determines auxininduced growth repression. Proc Natl Acad Sci U S A 113, 452-457, doi:10.1073/pnas.1517445113 (2016).

44 Muller, O. et al. Autophagic tubes: vacuolar invaginations involved in lateral membrane sorting and inverse vesicle budding. J Cell Biol 151, 519-528, doi:10.1083/jcb.151.3.519 (2000).

45 Allen, R. D. \& Naitoh, Y. Osmoregulation and contractile vacuoles of protozoa. Int Rev Cytol 215, 351-394, doi:10.1016/s0074-7696(02)15015-7 (2002).

46 Komsic-Buchmann, K., Stephan, L. M. \& Becker, B. The SEC6 protein is required for contractile vacuole function in Chlamydomonas reinhardtii. J Cell Sci 125, 2885-2895, doi:10.1242/jcs.099184 (2012).

47 Nishiyama, T. et al. The Chara Genome: Secondary Complexity and Implications for Plant Terrestrialization. Cell 174, 448-464 e424, doi:10.1016/j.cell.2018.06.033 (2018). 
bioRxiv preprint doi: https://doi.org/10.1101/2022.01.18.476728; this version posted January $20,2022$. The copyright holder for this preprint (which was not certified by peer review) is the author/funder, who has granted bioRxiv a license to display the preprint in perpetuity. It is made available under aCC-BY-NC-ND 4.0 International license.

48 Persson, S. et al. The Arabidopsis irregular xylem8 mutant is deficient in glucuronoxylan and homogalacturonan, which are essential for secondary cell wall integrity. Plant Cell 19, 237-255, doi:10.1105/tpc.106.047720 (2007).

49 Chiniquy, D. et al. Three Novel Rice Genes Closely Related to the Arabidopsis IRX9, IRX9L, and IRX14 Genes and Their Roles in Xylan Biosynthesis. Front Plant Sci 4, 83, doi:10.3389/fpls.2013.00083 (2013).

50 Zhang, B., Gao, Y., Zhang, L. \& Zhou, Y. The plant cell wall: Biosynthesis, construction, and functions. J Integr Plant Biol 63, 251-272, doi:10.1111/jipb.13055 (2021).

51 Avci, U., Earl Petzold, H., Ismail, I. O., Beers, E. P. \& Haigler, C. H. Cysteine proteases XCP1 and XCP2 aid micro-autolysis within the intact central vacuole during xylogenesis in Arabidopsis roots. Plant J 56, 303-315, doi:10.1111/j.1365-313X.2008.03592.x (2008).

52 Kuriyama, $\mathrm{H}$. Loss of Tonoplast Integrity Programmed in Tracheary Element Differentiation. Plant Physiol 121, 763-774 (1999).

53 Groover, A., DeWitt, N., Heidel, A. \& Jones, A. Programmed cell death of plant tracheary elements differentiating in vitro. Protoplasma Protoplasma, 197-211, doi:https://doi.org/10.1007/BF01279568 (1997).

54 Hara-Nishimura, I. \& Hatsugai, N. The role of vacuole in plant cell death. Cell death and differentiation 18, 1298-1304, doi:10.1038/cdd.2011.70 (2011).

55 Groover, A. \& Jones, A. M. Tracheary element differentiation uses a novel mechanism coordinating programmed cell death and secondary cell wall synthesis. Plant Physiol 119, 375-384, doi:10.1104/pp.119.2.375 (1999).

56 Courtois-Moreau, C. L. et al. A unique program for cell death in xylem fibers of Populus stem. Plant J 58, 260-274, doi:10.1111/j.1365-313X.2008.03777.x (2009).

57 Kwon, S. I. et al. The Rab GTPase RabG3b functions in autophagy and contributes to tracheary element differentiation in Arabidopsis. Plant J 64, 151-164, doi:10.1111/j.1365313X.2010.04315.x (2010).

58 Herman, E. \& Schmidt, M. Endoplasmic reticulum to vacuole trafficking of endoplasmic reticulum bodies provides an alternate pathway for protein transfer to the vacuole. Plant Physiol 136, 3440-3446, doi:10.1104/pp.104.051722 (2004).

59 Michaeli, S., Avin-Wittenberg, T. \& Galili, G. Involvement of autophagy in the direct ER to vacuole protein trafficking route in plants. Front Plant Sci 5, 134, doi:10.3389/fpls.2014.00134 (2014).

60 Toyooka, K., Okamoto, T. \& Minamikawa, T. Mass transport of proform of a KDEL-tailed cysteine proteinase (SH-EP) to protein storage vacuoles by endoplasmic reticulumderived vesicle is involved in protein mobilization in germinating seeds. J Cell Biol 148, 453-464, doi:10.1083/jcb.148.3.453 (2000).

61 Okamoto, T., Shimada, T., Hara-Nishimura, I., Nishimura, M. \& Minamikawa, T. Cterminal KDEL sequence of a KDEL-tailed cysteine proteinase (sulfhydryl-endopeptidase) is involved in formation of KDEL vesicle and in efficient vacuolar transport of sulfhydrylendopeptidase. Plant Physiol 132, 1892-1900, doi:10.1104/pp.103.021147 (2003).

62 Lee, J. Y. et al. Transcriptional and posttranscriptional regulation of transcription factor expression in Arabidopsis roots. Proc Natl Acad Sci U S A 103, 6055-6060, doi:10.1073/pnas.0510607103 (2006).

63 Olvera-Carrillo, Y. et al. A Conserved Core of Programmed Cell Death Indicator Genes Discriminates Developmentally and Environmentally Induced Programmed Cell Death in Plants. Plant Physiol 169, 2684-2699, doi:10.1104/pp.15.00769 (2015).

64 Segami, S., Makino, S., Miyake, A., Asaoka, M. \& Maeshima, M. Dynamics of vacuoles and $\mathrm{H}+$-pyrophosphatase visualized by monomeric green fluorescent protein in Arabidopsis: artifactual bulbs and native intravacuolar spherical structures. Plant Cell 26, 3416-3434, doi:10.1105/tpc.114.127571 (2014).

65 Dettmer, J., Hong-Hermesdorf, A., Stierhof, Y. D. \& Schumacher, K. Vacuolar H+-ATPase activity is required for endocytic and secretory trafficking in Arabidopsis. Plant Cell 18, 715-730, doi:10.1105/tpc.105.037978 (2006). 
66 Paredez, A. R., Somerville, C. R. \& Ehrhardt, D. W. Visualization of cellulose synthase demonstrates functional association with microtubules. Science 312, 1491-1495, doi:10.1126/science.1126551 (2006).

67 Jaillais, Y., Belkhadir, Y., Balsemao-Pires, E., Dangl, J. L. \& Chory, J. Extracellular leucine-rich repeats as a platform for receptor/coreceptor complex formation. Proc Natl Acad Sci U S A 108, 8503-8507, doi:10.1073/pnas.1103556108 (2011).

68 Geldner, N. et al. Rapid, combinatorial analysis of membrane compartments in intact plants with a multicolor marker set. Plant $J$ 59, 169-178, doi:10.1111/j.1365313X.2009.03851.x TPJ3851 [pii] (2009).

69 Vermeer, J. E. M. et al. A Spatial Accommodation by Neighboring Cells Is Required for Organ Initiation in Arabidopsis. Science 343, 178-183, doi:10.1126/science.1245871 (2014).

70 Chauvin, A., Caldelari, D., Wolfender, J. L. \& Farmer, E. E. Four 13-lipoxygenases contribute to rapid jasmonate synthesis in wounded Arabidopsis thaliana leaves: a role for lipoxygenase 6 in responses to long-distance wound signals. New Phytol 197, 566-575, doi:10.1111/nph.12029 (2013).

71 Curtis, M. D. \& Grossniklaus, U. A gateway cloning vector set for high-throughput functional analysis of genes in planta. Plant Physiol 133, 462-469, doi:10.1104/pp.103.027979 (2003).

72 Ursache, R., Andersen, T. G., Marhavy, P. \& Geldner, N. A protocol for combining fluorescent proteins with histological stains for diverse cell wall components. Plant J 93, 399-412, doi:10.1111/tpj.13784 (2018).

73 Mahonen, A. P. et al. A novel two-component hybrid molecule regulates vascular morphogenesis of the Arabidopsis root. Genes Dev 14, 2938-2943 (2000).

74 Brzeska, H., Guag, J., Remmert, K., Chacko, S. \& Korn, E. D. An experimentally based computer search identifies unstructured membrane-binding sites in proteins: application to class I myosins, PAKS, and CARMIL. J Biol Chem 285, 5738-5747, doi:10.1074/jbc.M109.066910 (2010).

75 Erdos, G. \& Dosztanyi, Z. Analyzing Protein Disorder with IUPred2A. Curr Protoc Bioinformatics 70, e99, doi:10.1002/cpbi.99 (2020).

76 Meszaros, B., Erdos, G. \& Dosztanyi, Z. IUPred2A: context-dependent prediction of protein disorder as a function of redox state and protein binding. Nucleic Acids Res 46, W329-W337, doi:10.1093/nar/gky384 (2018). 
bioRxiv preprint doi: https://doi.org/10.1101/2022.01.18.476728; this version posted January 20, 2022. The copyright holder for this FIGURE 1 preprint (which was not certified by peer review) is the author/funder, who has granted bioRxiv a license to display the preprint in

a
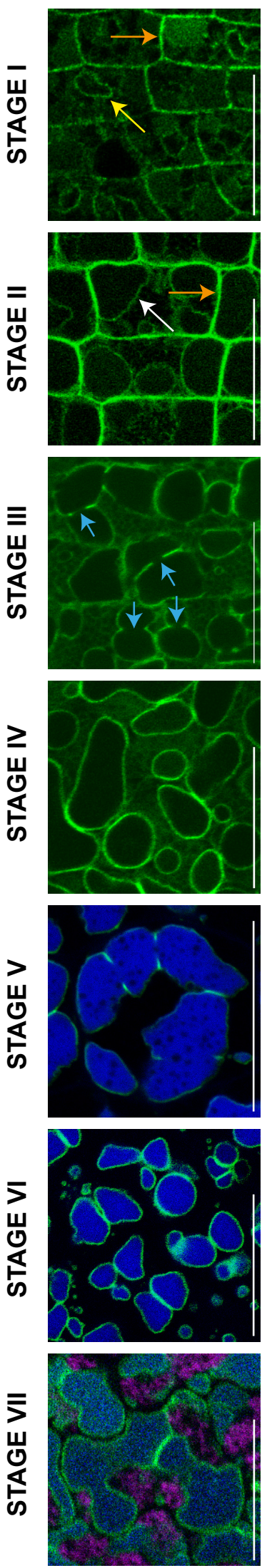

pten $2 a$ pten $2 b$
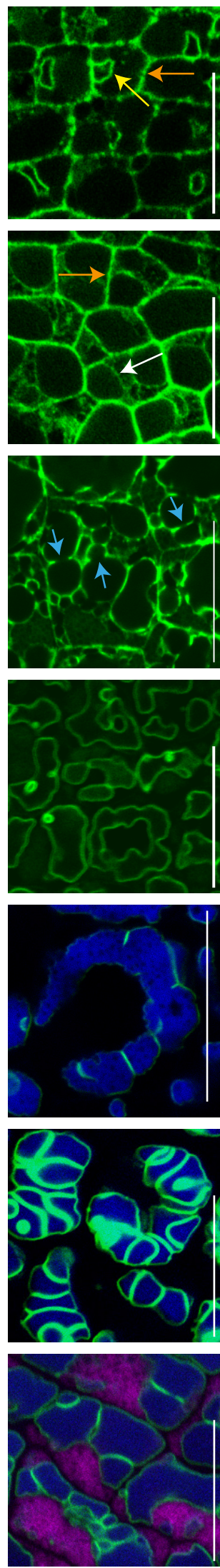

b WT
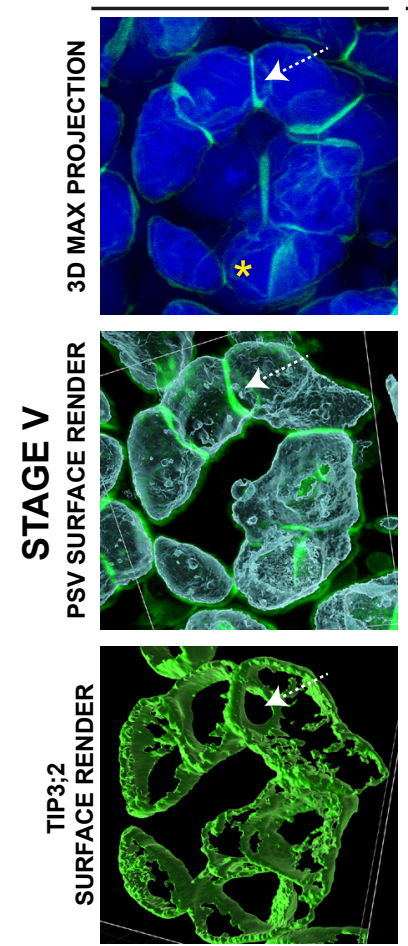

C
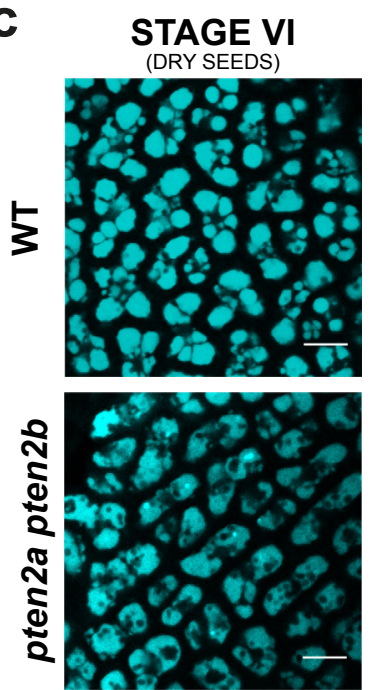

d

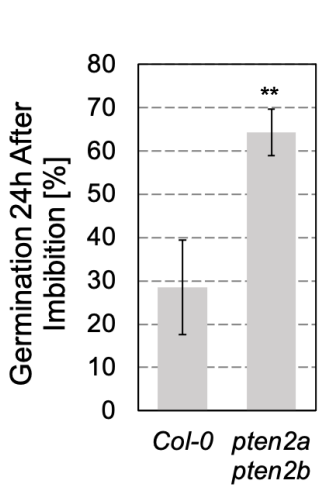

pten2a pten $2 b$
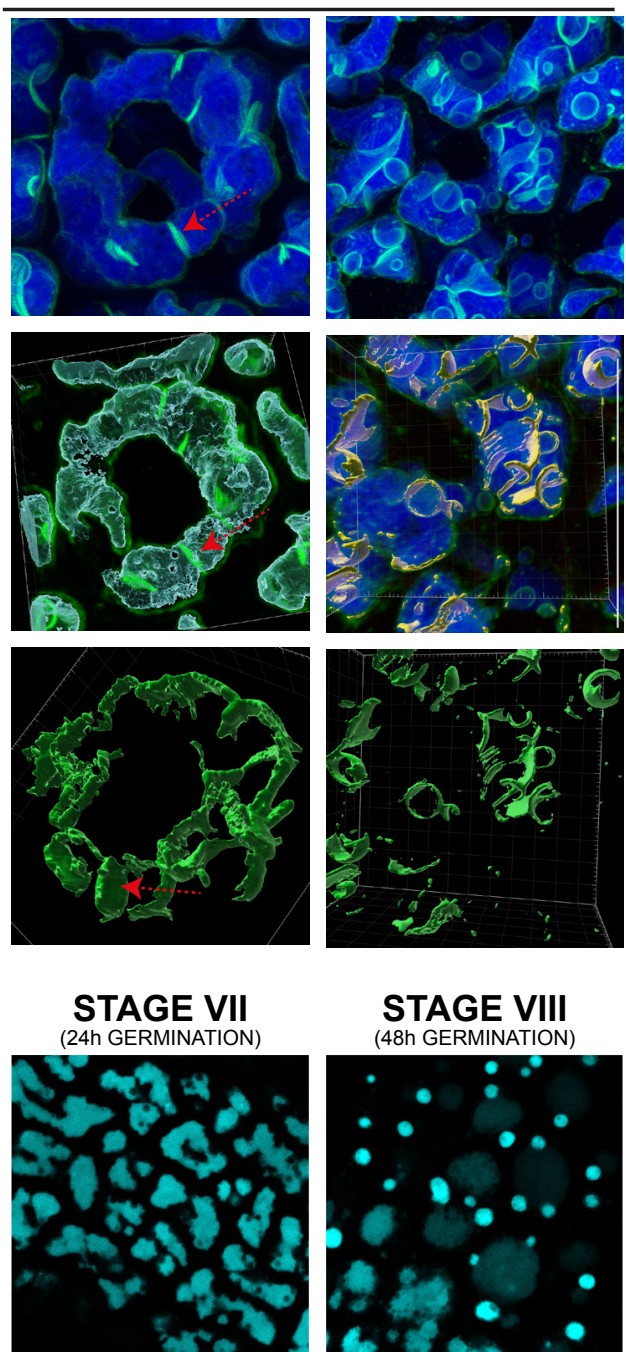

STAGE VIII
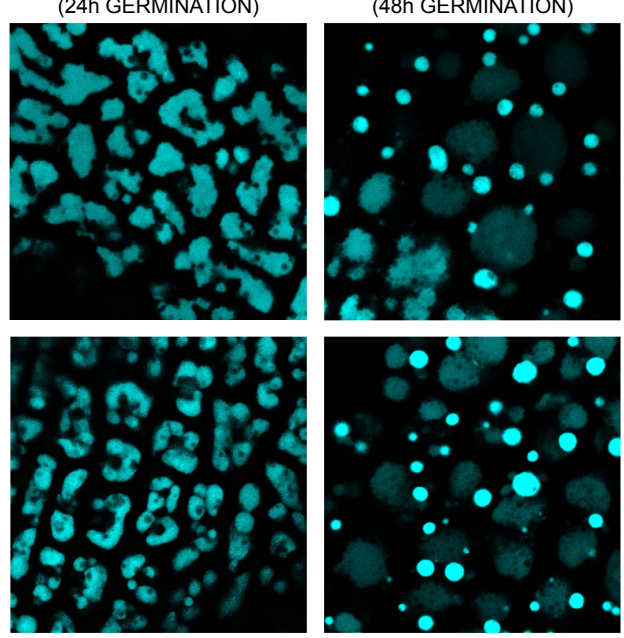

e

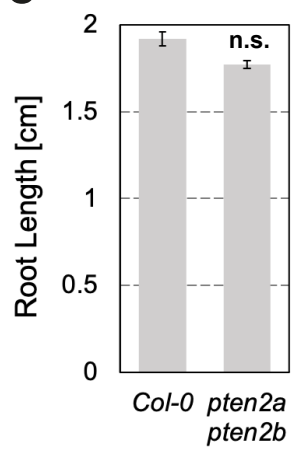

f

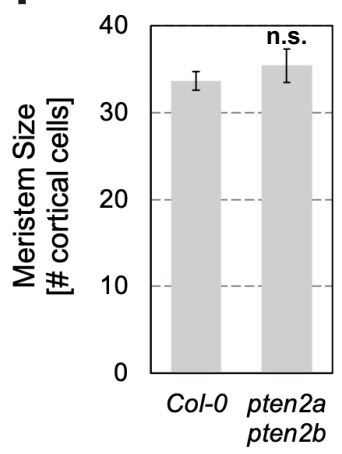

Figure 1: Embryonic vacuole division involves cleavage furrow formation and requires PTEN2 activity. a-h, Representative confocal images of vacuoles in epidermal cells in Arabidopsis embryos extracted from green siliques (stages I-III), yellow siliques (stages IV-V), dry seeds (VI), germinated for 24h (stage VII) or 48h (stage VIII). Tonoplast (vacuolar membrane) decorated by TIP3;2-GFP (in green). a, Comparative seven stages of TIP3;2 dynamics between wild type and pten2a pten2b. At the onset of its expression, TIP3;2 accumulates in endoplasmic reticulum close to plasma membrane (orange arrows), or follow nuclear shape (yellow arrows). First tonoplast is visible in stage II marked with a white arrow. Small pre-embryonic vacuoles fuse in stage III (fusion sites are marked with blue arrows). Please note that image of EVs in pten2a pten $2 b$ in stage IV is slightly advanced than in wild type. In stages V-VII PSV exhibit autofluorescence in blue part of the spectrum (represented blue in images). Magenta shows autofluorescence in red part of the spectrum. b, EV division starts by tonoplast invagination at division site visible as a circle (white dashed arrows). In addition to the lack of synchronicity in tonoplast invaginations, in pten2a pten $2 b$ double mutants, membrane unilaterally ingress (red dashed arrow) and can reach the other side of the vacuole, however the division does not occur. Possible cause is the absence of membrane bending into hourglass shape as visible in WT (yellow asterisk). The ingrown membrane possibly rolls into a cylindrical shape as visible in 3D reconstructions of the late-stage $\mathrm{V}$ in double mutants. c, Representative confocal images of storage vacuoles' autofluorescence in epidermal cells of Arabidopsis embryos extracted from dry seeds and germinating seedlings in wild type and pten2a pten2b mutants. Scale bars represent $20 \mu \mathrm{m}$. d, Germination $24 \mathrm{~h}$ after imbibition in indicated genotypes, $\mathrm{n}>500$. e-f, Quantification of root length (e) and meristem size (f) in 6 days old seedlings of the indicated genotypes. $n>30$ (e) and $n=10$ (f). All error bars represent standard error. $n . s .$, not significant; ${ }^{* *} p<0.01$. 
bioRxiv preprint doi: https://doi.org/10.1101/2022.01.18.476728; this version posted January 20, 2022. The copyright holder for this EXTENDEDeDATARiqVUREeryfied by peer review) is the author/funder, who has granted bioRxiv a license to display the preprint in

a

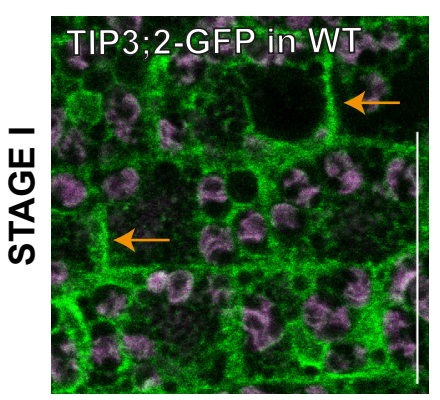

b

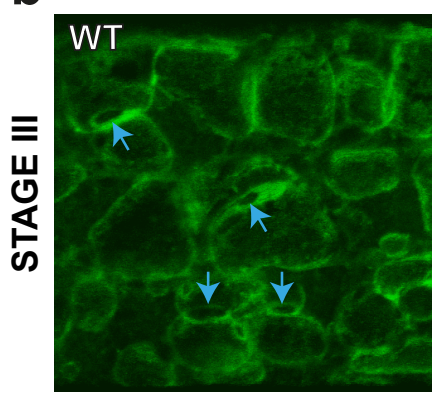

C

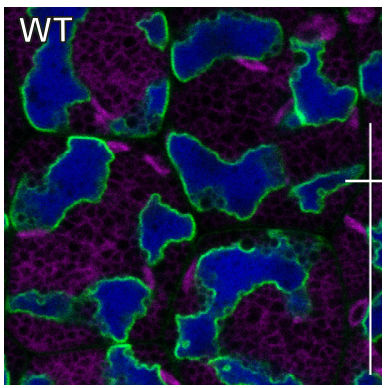

d

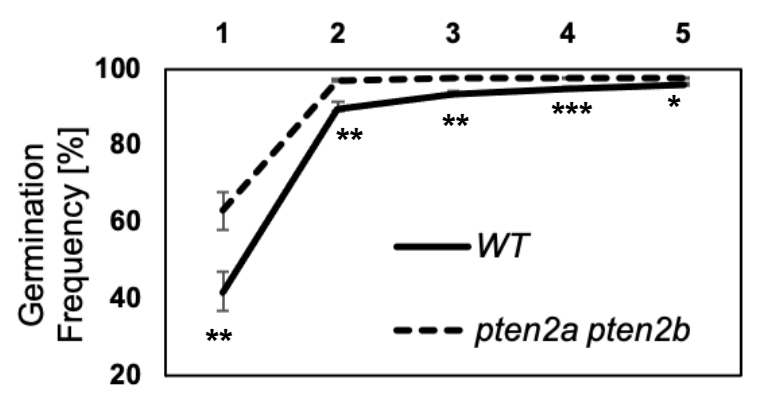

$\mathbf{e}$

PTEN2b Relative Expression

$\begin{array}{llllll}0 & 50 & 100 & 150 & 200 & 250\end{array}$
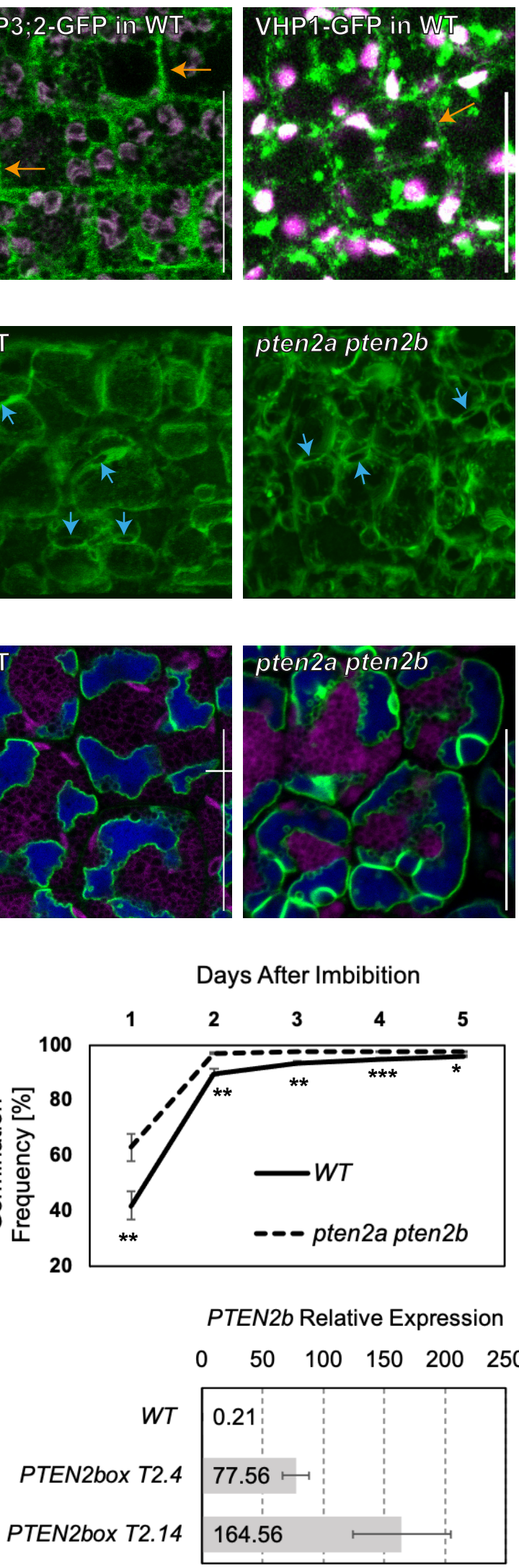

f
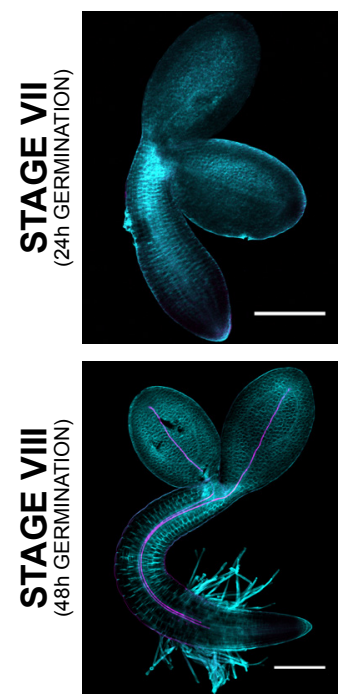

pten $2 a$ pten $2 b$

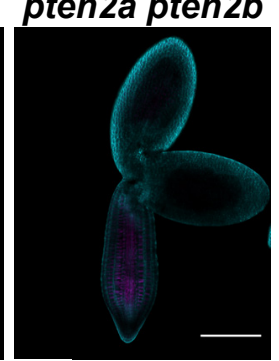

PTEN2box
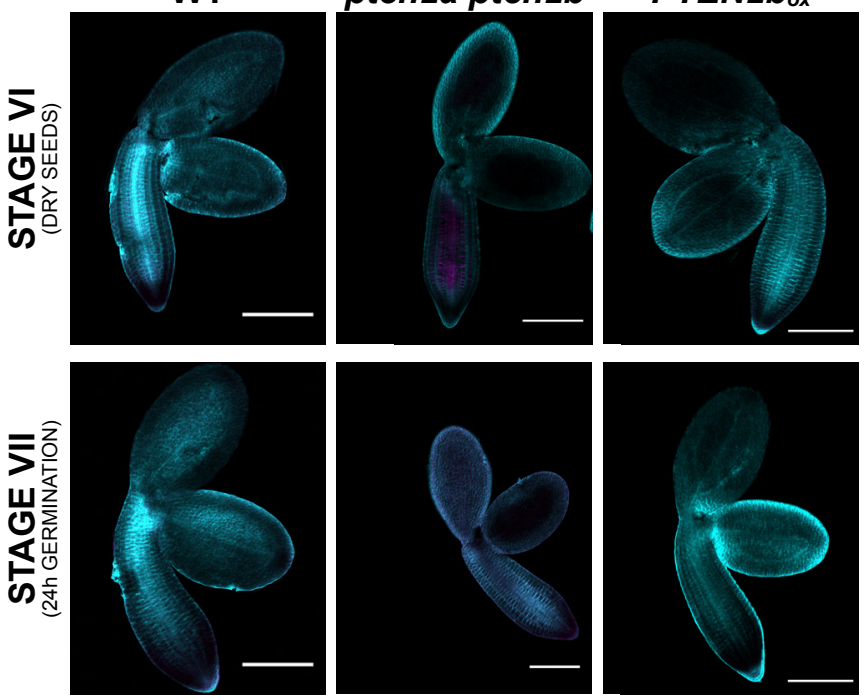

g
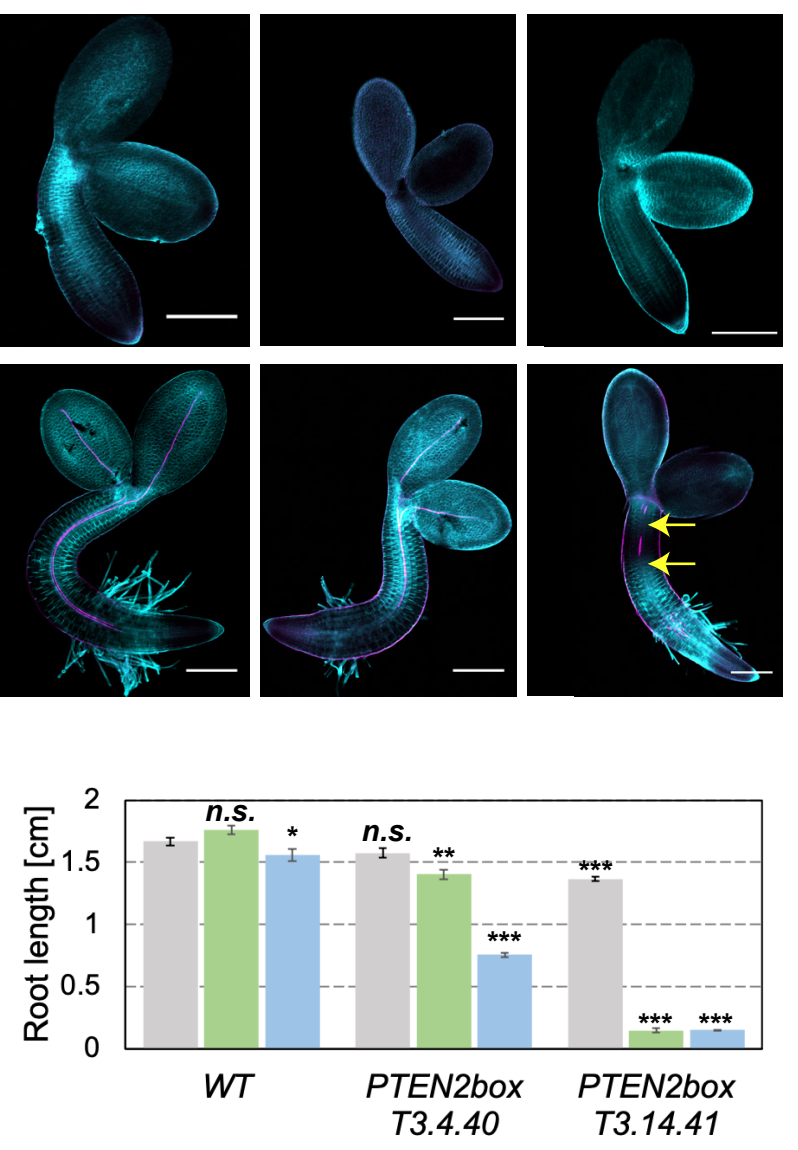

$\square$ mock $\quad 0.2 \mu \mathrm{M}$ estradiol $\quad \square 2 \mu \mathrm{M}$ estradiol

h

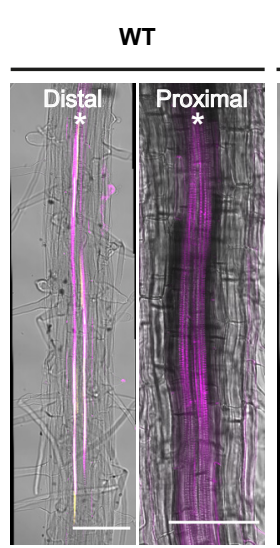

UBQ::XVE::PTEN2b T3.4.40

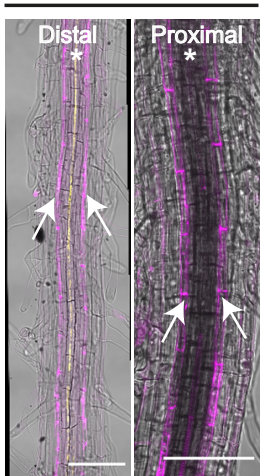

UBQ::XVE:::PTEN2b T3.14.41

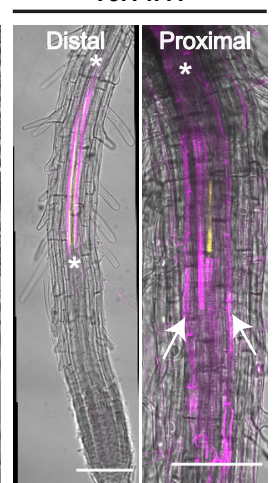

Extended Data Figure 1: Aberrant EV division does not affect post-embryonic development. a-c, Representative confocal images of aquaporin TIP3;2-GFP (green) distribution during embryogenesis. a, Similar to TIP3;2, VHP1 tonoplast marker also labels ER. Magenta shows autofluorescence from chloroplasts visible in embryos isolated from green siliques. b, 3D maximal projection of a stage III vacuole corresponding to $2 \mathrm{D}$ image shown in Figure 1a. c, Small vacuoles labelled with TIP3;2 close to EV tonoplast preceding the cleavage furrow division. Magenta shows autofluorescence detected in red part of the spectrum. Scale bars represent $20 \mu \mathrm{m}$. d, Germination rate between indicated genotypes. Error bars represent SE. $\mathrm{n}>500$. e, Analysis of normalized, relative PTEN2b overexpression by qRT-PCR in two independent transgenic lines induced with $2 \mu \mathrm{M}$ estradiol for the duration of 6 days. Error bars represent SE among three independent biological replicates. $f$, Representative confocal images of embryos dissected from dry seeds, $24 \mathrm{~h}$ and $48 \mathrm{~h}$ after imbibition of the indicated genotypes, stained with Calcofluor White (cellulose in cyan) and fuchsin (lignin in magenta). Yellow arrows mark xylem discontinuities in seedlings with induced PTEN2b overexpression from imbibition $(2 \mu \mathrm{M}$ estradiol). Note the appearance of differentiated xylem cells (magenta) only $48 \mathrm{~h}$ after germination. Scale bars 200 $\mu \mathrm{m}$. g, Root length quantification of 6-days-old seedlings illustrate dose dependant effect of PTEN2b overexpression after 48h of estradiol induction. Error bars represent SE. $n>40 \mathrm{~h}$, PTEN2b overexpression prevents xylem differentiation in T3.4.40 line in both proximal and distal part of the root. Higher overexpression in the line T3.13.41 dramatically shortens the root length $(\mathrm{g})$ but does not prevent xylem differentiation in younger distal root parts. Undifferentiated xylem is only labelled with MYB46 marker in yellow, while differentiated xylem is labelled with fuchsin staining for lignin in magenta, or white (overlap between yellow and magenta). Asterisk labels xylem position within vascular cylinder. White arrows label ectopic lignification in endodermis. Scale bars represent $100 \mu m$. n.s., not significant; ${ }^{*} p<0.05 ;{ }^{* *} p<0.01 ;{ }^{* * *} p<0.001$ 
bioRxiv preprint doi: https://doi.org/10.1101/2022.01.18.476728; this version posted January 20, 2022. The copyright holder for this FIGURE 2 preprint (which was not certified by peer review) is the author/funder, who has granted bioRxiv a license to display the preprint in

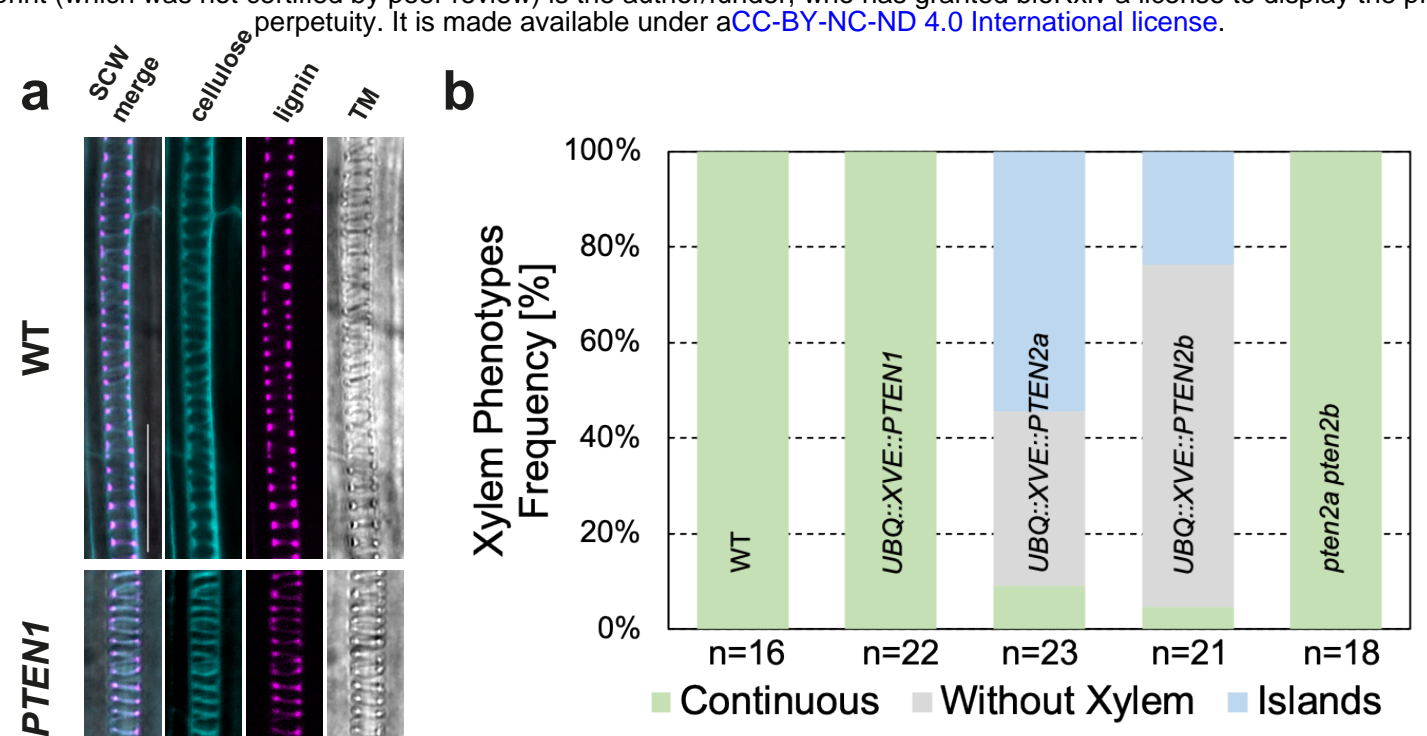

C
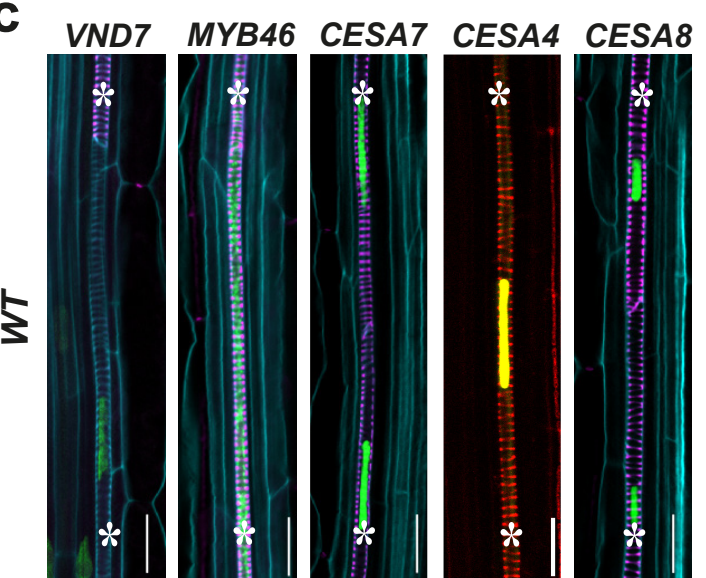

DMP4

\section{EXI1}

@̊
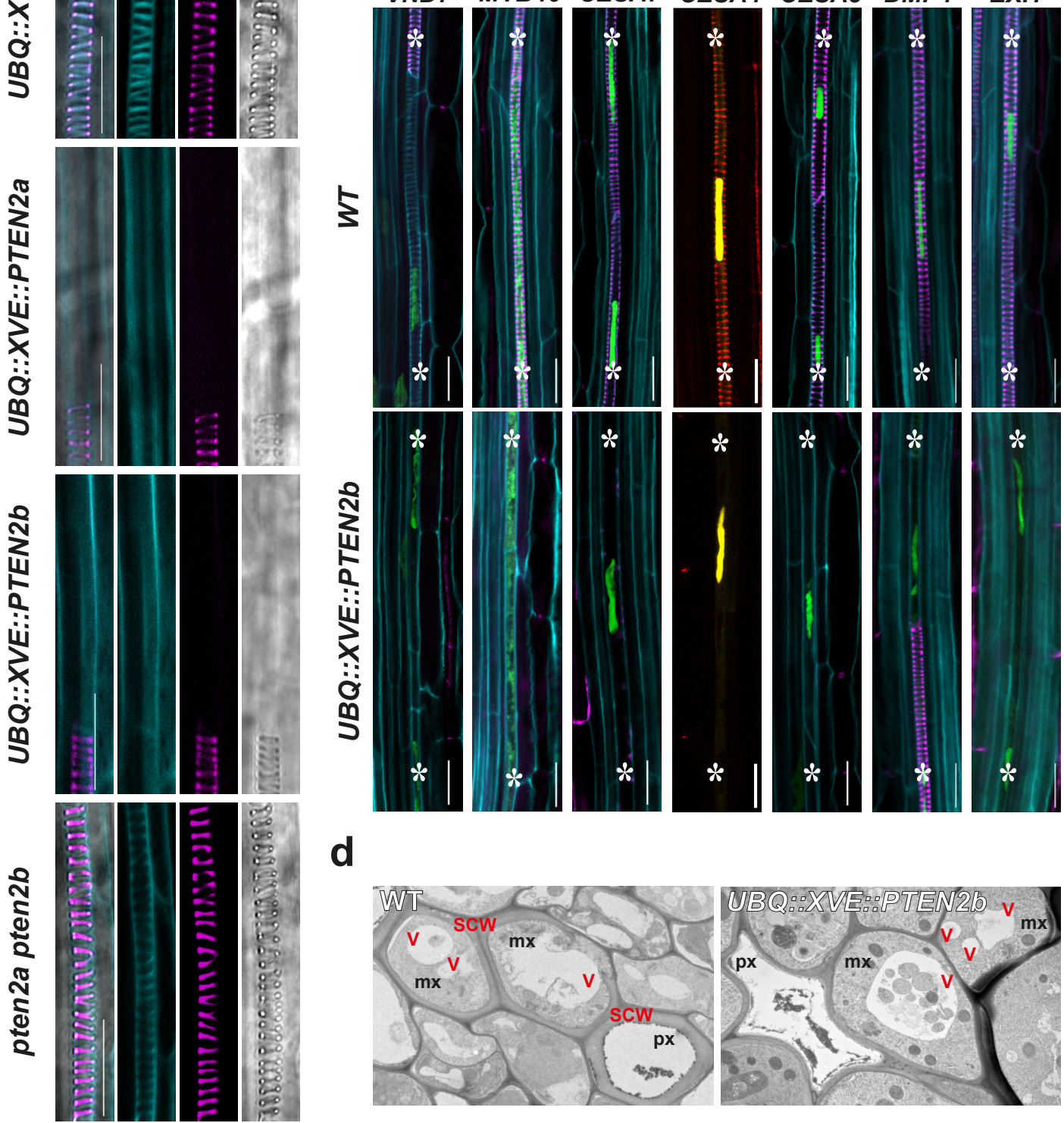

d

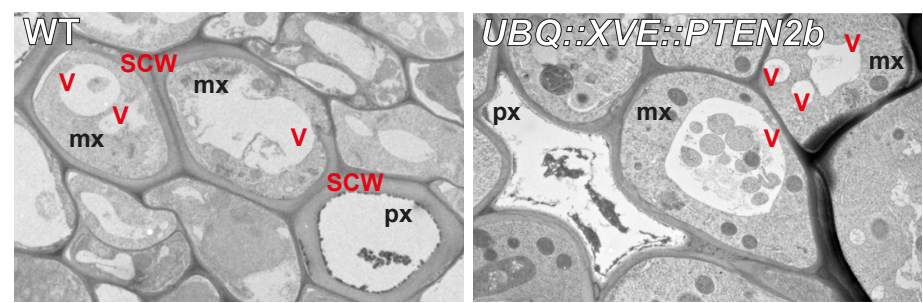

Figure 2: PTEN2s prevent xylem cell differentiation. a, Representative confocal images of protoxylem cells in the indicated genotypes stained with Calcofluor White (cellulose in cyan) and Fuchsin (lignin in magenta). Secondary cell wall (SCW) was also visualised by transmission light (TM). Scale bars represent $20 \mu \mathrm{m}$. b. Quantification of xylem phenotypes observed in the roots displayed in a. The two phenotypes observed and scored were the total absence of xylem strands (without xylem) or the appearance of several protoxylem cells with SCW (islands). c, Representative images of indicated xylem differentiation markers in wildtype (WT) and seedlings incubated in $0.2 \mu \mathrm{M}$ estradiol for $48 \mathrm{~h}$ to trigger PTEN2box. Roots were stained with Calcofluor White (cyan) and fuchsin (magenta). Marker lines: VND7 (VASCULAR RELATED NAC-DOMAIN PROTEIN 7), MYB46 (MYB DOMAIN PROTEIN 46), CESA7 (CELLULOSE SYNTHASE CATALYTIC SUBUNIT 7), CESA4 (CELLULOSE SYNTHASE A4), CESA8 (CELLULOSE SYNTHASE 8), DMP4 (DUF679 DOMAIN MEMBRANE PROTEIN 4), EXI1 (EXITUS 1). Scale bars represent $20 \mu \mathrm{m}$. d, Transmission electron microscopy images of differentiating proto- (px) and metaxylem (mx) cells in WT and PTEN2box. Xylem cells in WT formed thick secondary cell wall (SCW), vacuoles are enlarging in $\mathrm{mx}$ while $\mathrm{px}$ underwent programmed cell death. PTEN2b overexpression prevents SCW formation while $\mathrm{mx}$ cells contain multiple small vacuoles. Here px cell also underwent clearance. 
bioRxiv preprint doi: https://doi.org/10.1101/2022.01.18.476728; this version posted January 20, 2022. The copyright holder for this

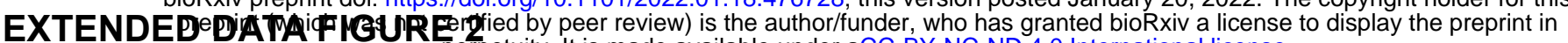
perpetuity. It is made available under aCC-BY-NC-ND 4.0 International license.

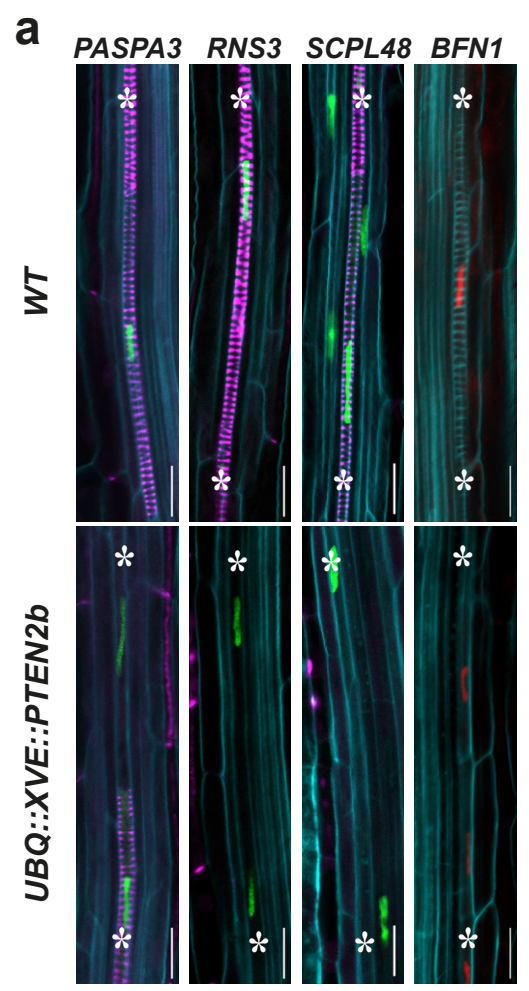

Extended Data Figure 2: PTEN2b overexpression does not alter the expression of genes associated with xylem PCD execution. a, Representative confocal microscopy images of the mature protoxylem cells stained with Calcofluor White for cellulose (cyan) and fuchsin for lignin (magenta). PTEN2b was induced for $48 \mathrm{~h}$ with $0.2 \mu \mathrm{M}$ estradiol. Note the expression of genes associated with PCD such as the PUTATIVE ASPARTIC PROTEINASE A3 (PASPA3), RIBONUCLEASE 3 (RNS3), SERINE CARBOXYPEPTIDASE-LIKE 48 (SCPL48) and BIFUNCTIONAL NUCLEASE 1 (BFN1) can still be detected in seedlings with PTEN2b upregulation. Asterisks mark protoxylem strands. Scale bars represent $20 \mu \mathrm{m}$. 
bioRxiv preprint doi: https://doi.org/10.1101/2022.01.18.476728; this version posted January 20,2022 . The copyright holder for this
FIGURE 3 preprint (which was not certified by peer review) is the author/funder, who has granted bioRxiv a license to display the preprint in perpetuity. It is made available under aCC-BY-NC-ND 4.0 International license.
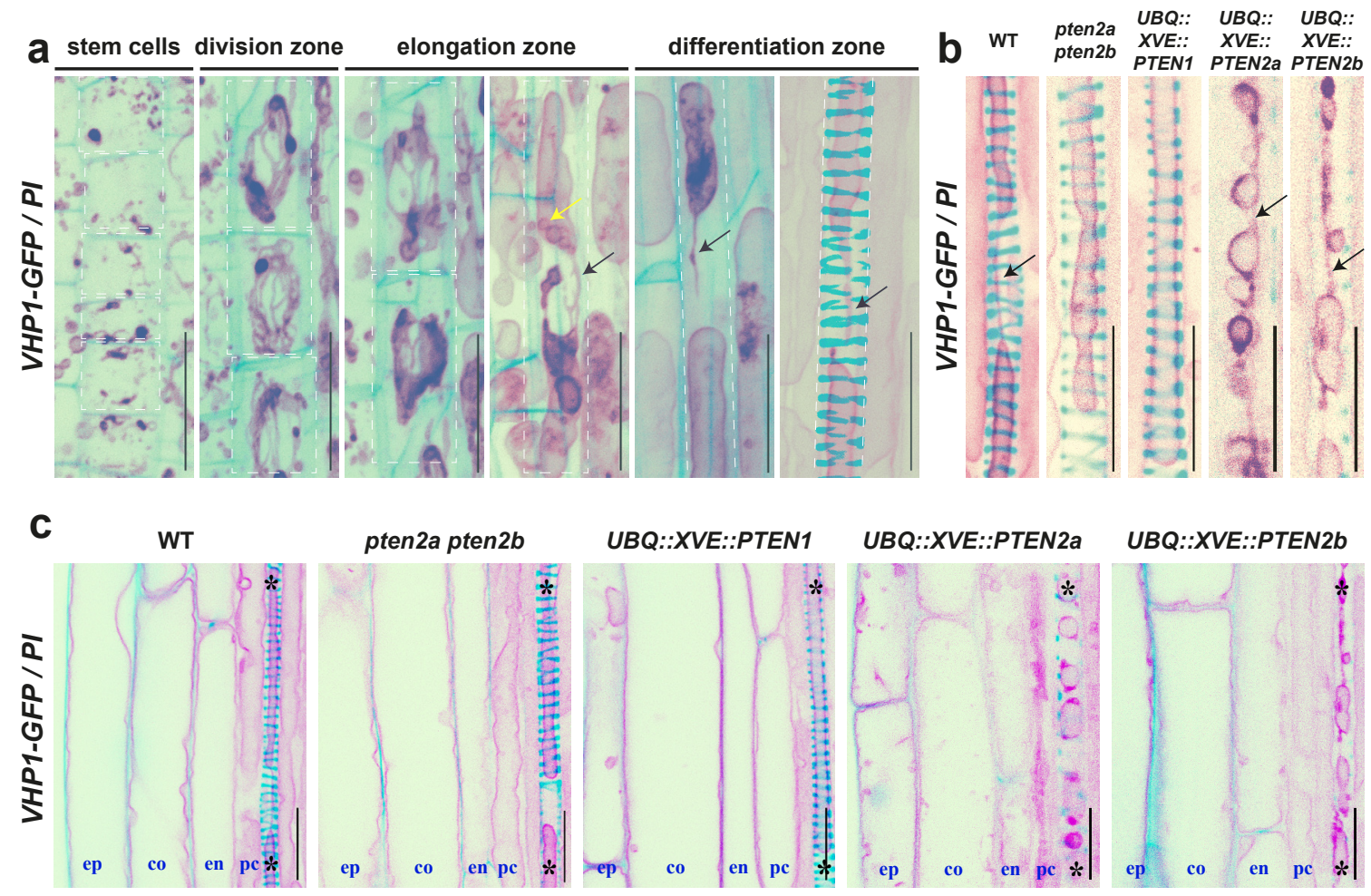

Figure 3: Regulation of xylem vacuolar biogenesis regulation by PTEN2s. a, 3D reconstruction of VHP1-GFP decorated vacuolar compartments in protoxylem cells in wild type plants at progressive developmental stages counterstained with propidium iodide $(\mathrm{PI})$ to label cell wall. Yellow arrows mark small vacuole-like compartments and black arrows mark tubular connecting membranes. For easier visualization, protoxylem cells margins were squared by a white dashed line. Scale bars represent $20 \mu \mathrm{m}$. b. Representative images of mature protoxylem cells in the indicated genotypes, visualized as in a. Scale bars represent $20 \mu \mathrm{m}$. Black arrows mark tubular connecting membranes. c, Comparison of vacuolar morphology in mature epidermis (ep), cortex (co), endodermis (en), pericycle (pc) and protoxylem (asterisk) between indicated genotypes. Scale bars represent $20 \mu \mathrm{m}$. 
bioRxiv preprint doi: https://doi.org/10.1101/2022.01.18.476728; this version posted January 20, 2022. The copyright holder for this FIGURE 4 preprint (which was not certified by peer review) is the author/funder, who has granted bioRxiv a license to display the preprint in perpetuity. It is made available under aCC-BY-NC-ND 4.0 International license.

a

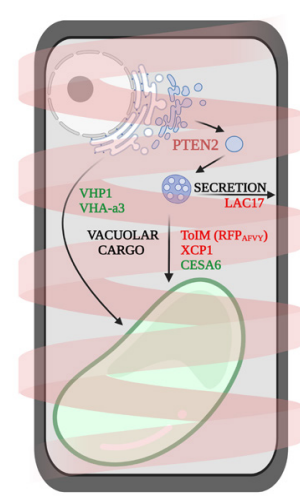

b WT PTEN2box

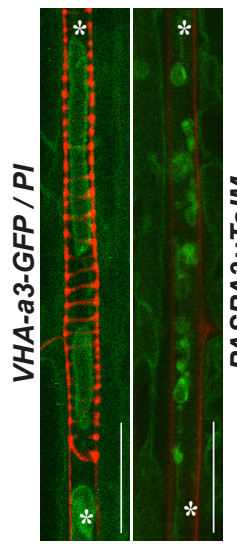

C d

d ${ }_{\text {WT PTEN2box }} \mathbf{e}$

e $\underset{\text { WT PTEN2box }}{\mathbf{f}}$

f ${ }_{\text {WT PTEN2box }} \mathbf{g}$ WT PTEN2box
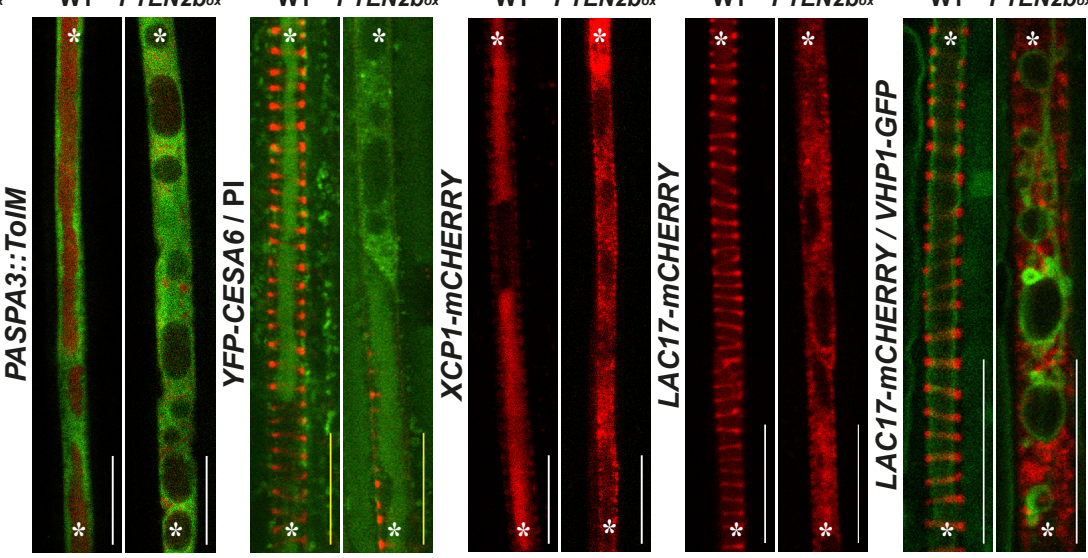

h WT

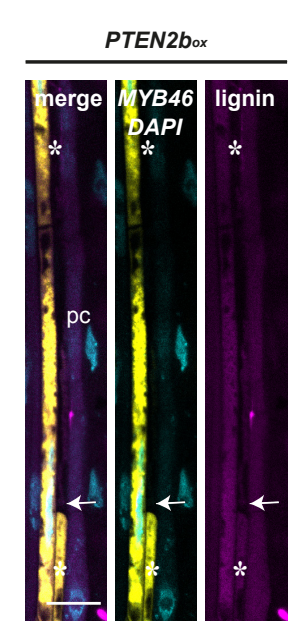

i
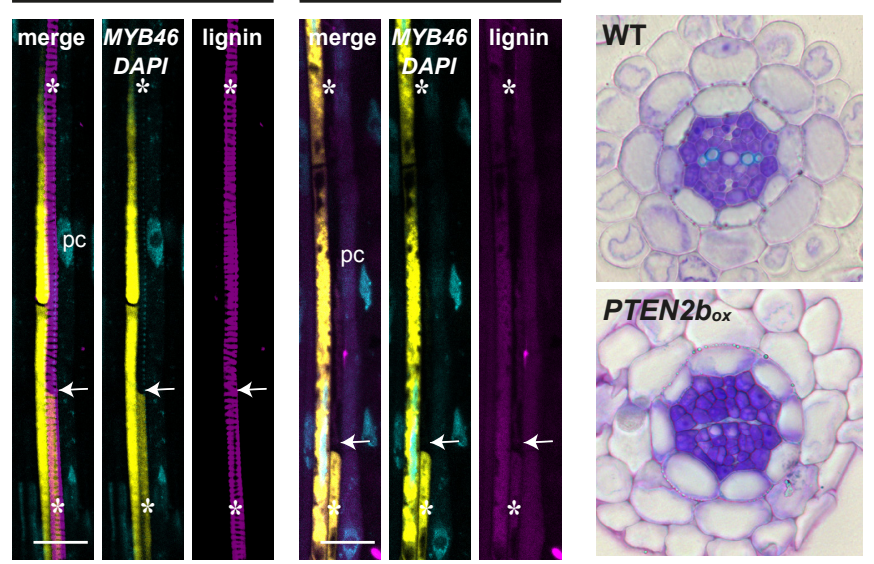

j

k
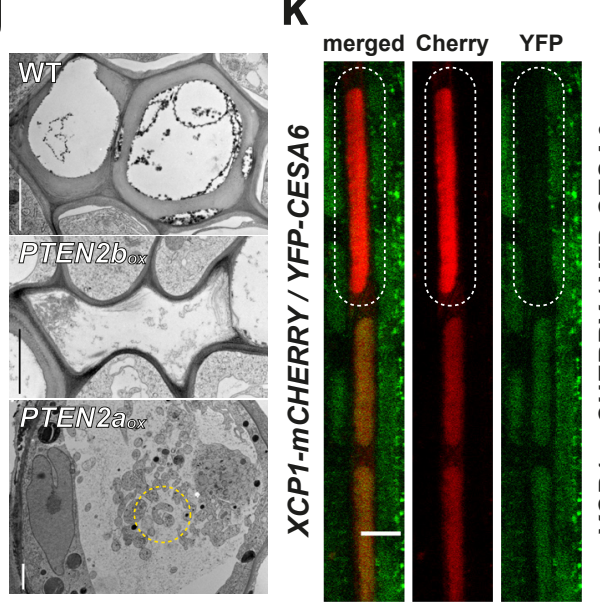

I

merged

Figure 4: PTEN2s inhibit vacuolar and secretion trafficking pathway in xylem cells but not PCD. a, Schematic representation of analysed trafficking pathways important for xylem cell differentiation. $\mathbf{b}-\mathbf{g}$, Representative images of the corresponding xylem cells in wild type (WT) and seedlings with $0.2 \mu \mathrm{M}$ estradiol-mediated $P T E N 2 b$ induction for $48 \mathrm{~h}$ visualizing different trafficking markers: tonoplast marker VHA-a3 (VACUOLAR PROTON ATPASE A3) (b), xylem specific promoter PASPA3 (PUTATIVE ASPARTIC PROTEINASE A3) driving expression of ToIM (tonoplast integrity marker) showing GFP in cytosol and a vacuolar targeted mRFP (c), vacuolar cargos CESA6 (CELLULOSE SYNTHASE SUBUNIT A6) (d) and XCP1 (XYLEM CYSTEINE PEPTIDASE 1) (e), secreted cargo LAC17 (LACCASE 17) (f). g, non-secreted LAC17 is not delivered into VHP1-labeled vacuoles. $\mathbf{h}-\mathbf{j}$, PCD execution occurs even without SCW formation in PTEN2box. $\mathbf{h}$, DAPI-stained nuclei are absent in the cells where the xylem-specific expression of MYB46 ceased due to the PCD execution (white arrows). Scale bars represent $20 \mu \mathrm{m}$. i, Toluidine-stained root cross-sections of the indicated genotypes. Xylem secondary cell wall stains bright blue as visible in WT but absent in PTEN2box overexpression where xylem cells appear collapsed as in transmission electron microscopy images (j). j, Transmission electron microscopy images of the indicated genotypes. Notice a high number of small vacuoles and aggregates in PTEN2box. Yellow dashed circle highlights a cup-shaped phagophore. Scale bars represent $2 \mu \mathrm{m}$. $\mathbf{k}$, Xylem vacuole acidification in wild type prior PCD. Note faster fading of $\mathrm{pH}$-sensitive YFP in comparison to $\mathrm{pH}$-tolerant mCHERRY. The cell where acidification occurs is encircled with a white dashed line. Scale bars represent $10 \mu \mathrm{m}$. I, continuation cell from $\mathrm{k}$, where PCD is executed and $\mathrm{mCHERRY} \mathrm{signal} \mathrm{disappears}$ too. Scale bars represent $10 \mu \mathrm{m}$. 

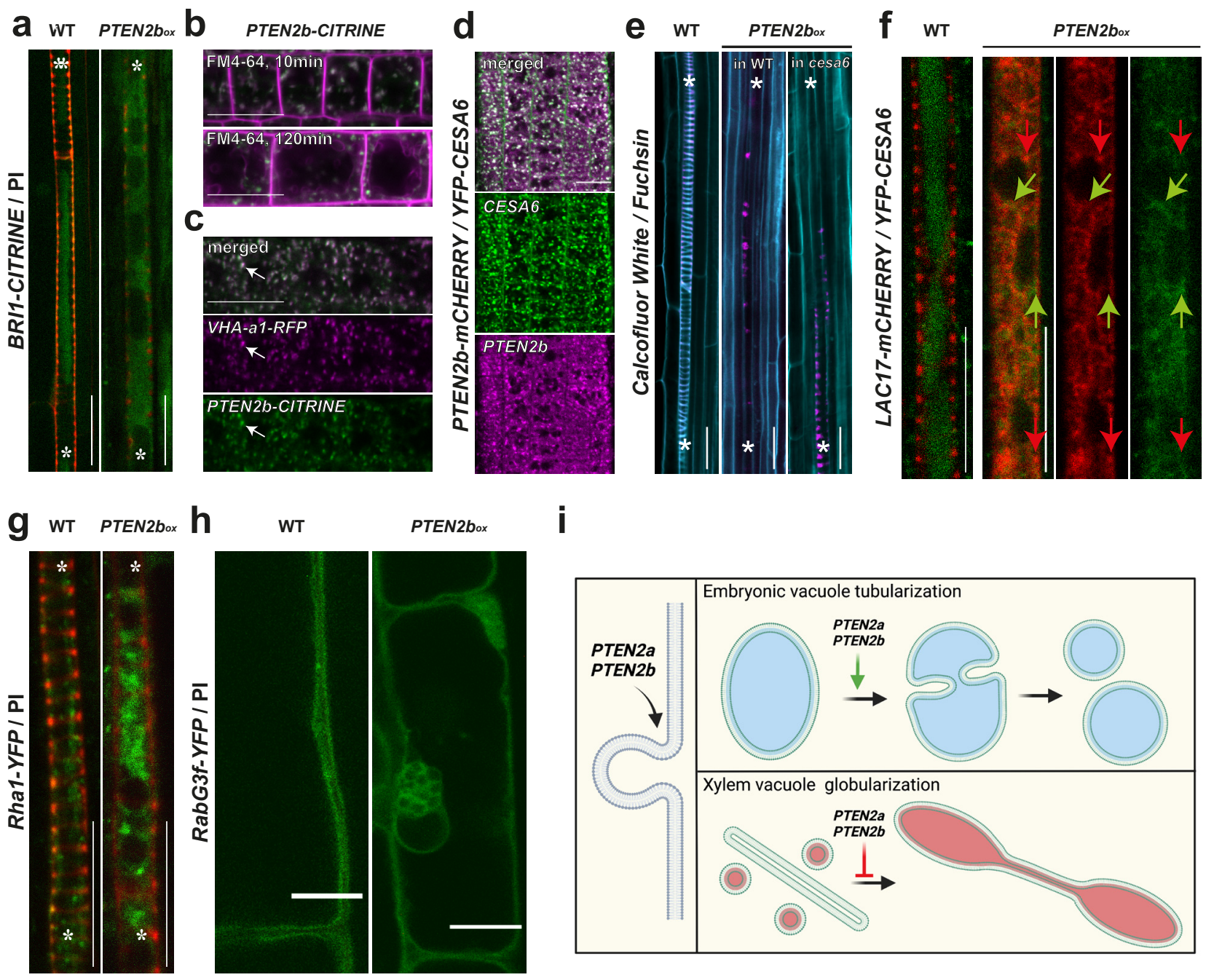

PTEN2box

i
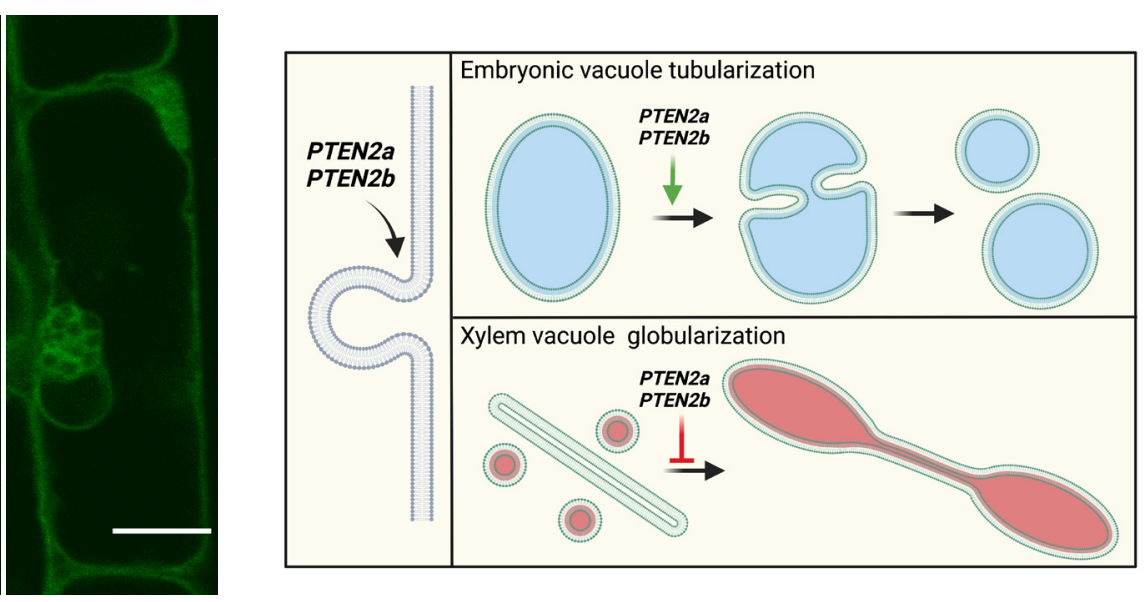

Extended Data Figure 4: PTEN2b colocalize to TGN and impinges on vacuolar and cellular secretion pathways. a, Brassinosteroid receptor BRI1 (BRASSINOSTEROID INSENSITIVE 1) cannot be delivered to xylem vacuoles upon PTEN2b overexpression. Seedlings were counterstained with propidium iodide (PI). b, PTEN2b colocalize with cellular compartments early labelled with FM4-64 (magenta). c, PTEN2b partially colocalize with PROTON ATPASE A1 (VHA-a1) in TGN (arrows). d, PTEN2b partially colocalize with CELLULOSE SYNTHASE SUBUNIT A6 (CESA6). e, cesa6 mutant cannot rescue secondary cellulose building upon PTEN2b overexpression. f, Aggregates of vesicles carrying vacuolar destined cargo (CESA6 in green) and secretion cargo (LAC17 in red) do not colocalize. Arrows' color corresponds to fluorophores and points the aggregates where proteins do not colocalize $\mathbf{g}$, Multivesicular body (MVB) marker Rha1 (ARABIDOPSIS RAB HOMOLOG F2A) creates aggregates in xylem cells upon PTEN2b upregulation. $\mathbf{h}$, Prevacuolar compartment and tonoplast marker RabG3f (RAB GTPASE HOMOLOG G3F) upon prolonged PTEN2b overexpression creates grape like structures in vicinity of the central vacuole in mature epidermal cells. Asterisk labels xylem strands. Scale bars represent $20 \mu \mathrm{m}$. i, Schematic representation of membrane phenomena regulated by PTEN2s. 
bioRxiv preprint doi: https://doi.org/10.1101/2022.01.18.476728; this version posted January 20, 2022. The copyright holder for this preprint (which was not certified by peer review) is the author/funder, who has granted bioRxiv a license to display the preprint in perpetuity. It is made available under aCC-BY-NC-ND 4.0 International license.

\section{FIGURE 5}

a

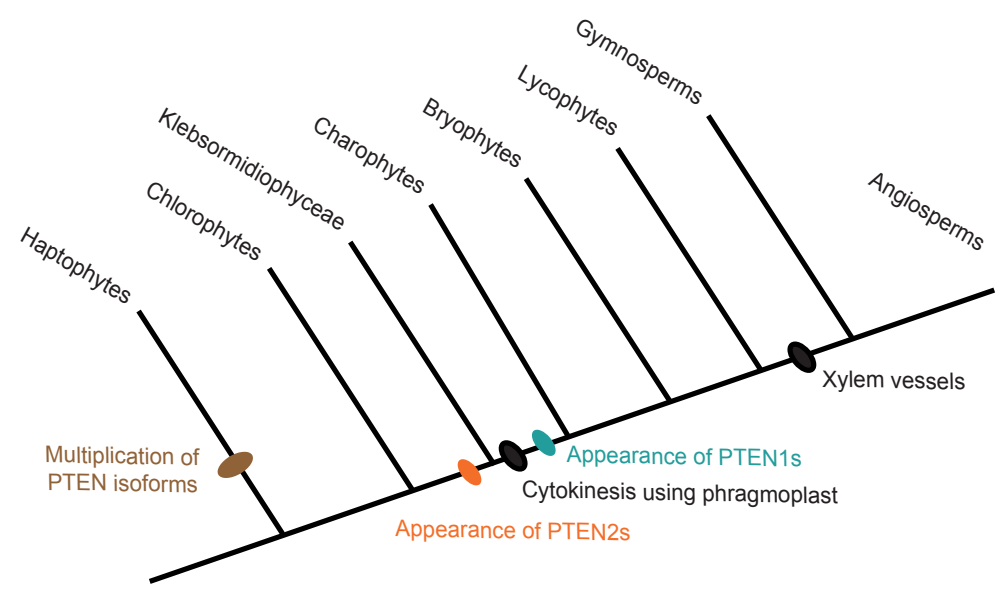

C

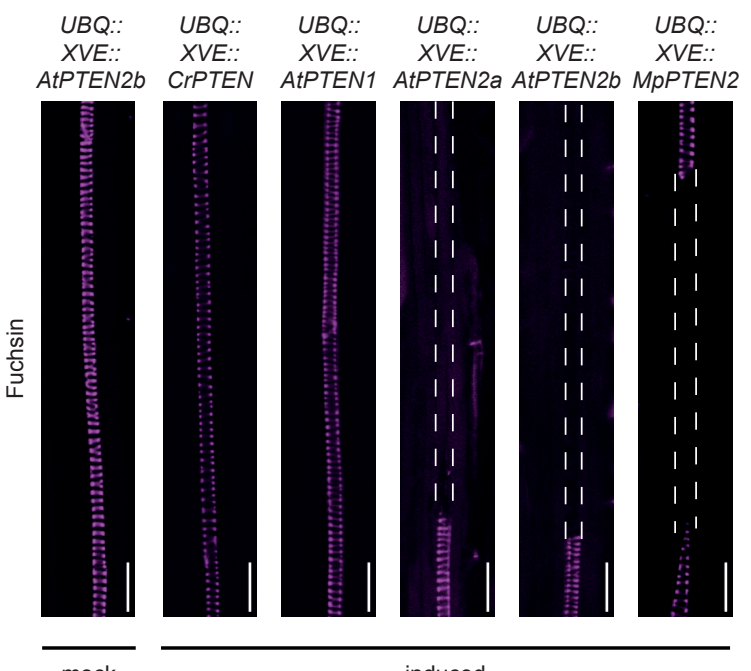

b

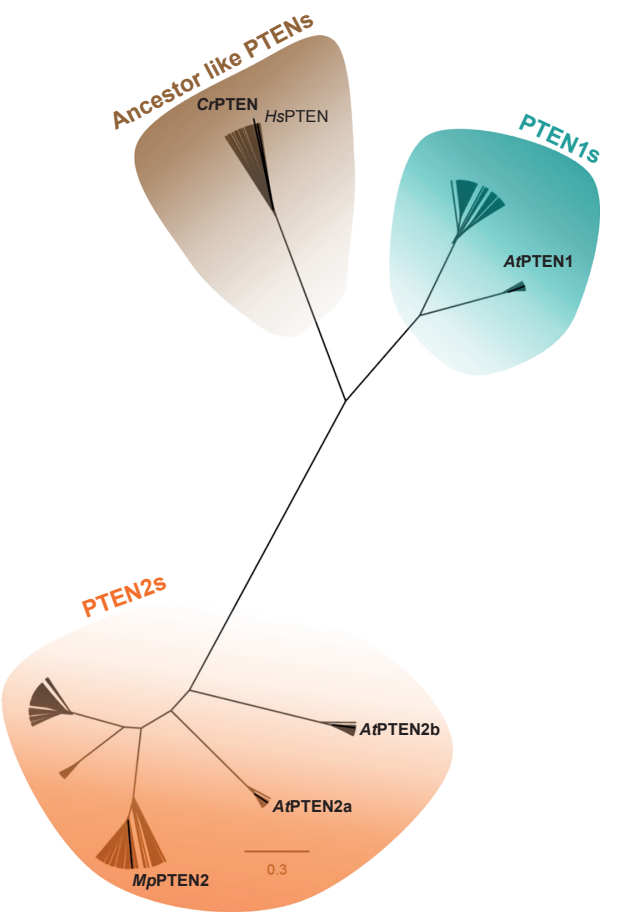

d

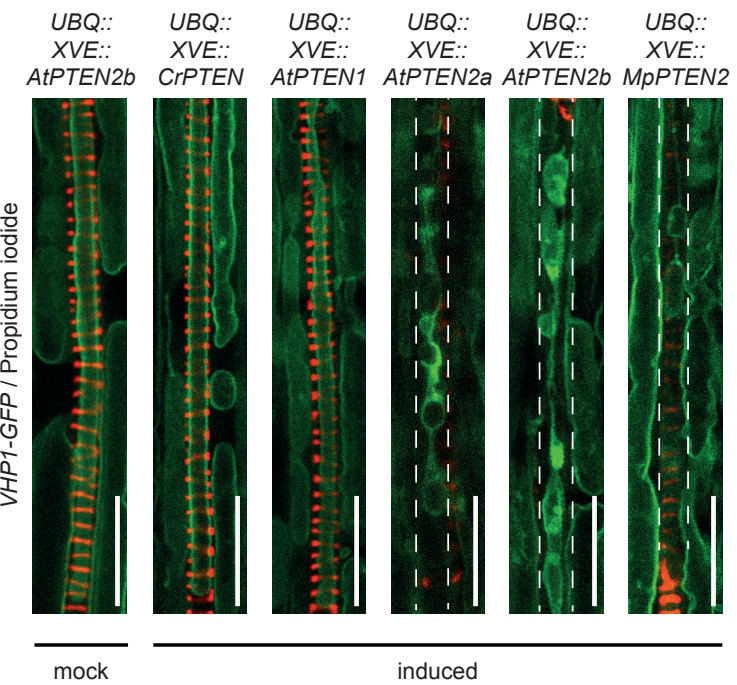

Figure 5: PTEN2s functions in vacuolar fusion and xylem differentiation were conserved through evolution. a, Schematic tree of the evolution of plant PTEN subfamilies. b. Phylogenetic tree of 418 plant PTENs from 142 plant species. For simplification, only the isoforms of species of interest have been represented. Details about all the sequences and the complete distribution of the isoforms in the three sub-families (ancestor-like PTENs, PTEN1s, PTEN2s) can be found in Supplementary Table 1. c, Representative confocal microscopy images of fuchsin-stained protoxylem strands from roots grown on mock conditions or upon $2 \mu \mathrm{M}$ estradiol-mediated induction for $48 \mathrm{~h}$ of $\mathrm{Chlamydomonas}$ reinharditi PTEN (CrPTEN), Arabidopsis thaliana PTEN1 (AtPTEN1), PTEN2a (AtPTEN2a), and PTEN2b (AtPTEN2b), Marchantia polymorpha PTEN2 (MpPTEN2). d, Representative confocal microscopy images of vacuolar morphology in mature xylem cells (VHP1-GFP labels tonoplast, propidium iodide stains cell wall) of inducible over-expressor lines of the different PTEN isoforms mentioned above. PTEN overepresion was induced for $48 \mathrm{~h}$ with $2 \mu \mathrm{M}$ estradiol. Protoxylem gaps are highlighted with white dashed lines. Scale bars represent $20 \mu \mathrm{m}$. 
bioRxiv preprint doi: https://doi.org/10.1101/2022.01.18.476728; this version posted January 20, 2022. The copyright holder for this

preprint (which was not certified by peer review) is the author/funder, who has granted bioRxiv a license to display the preprint in perpetuity. It is made available under aCC-BY-NC-ND 4.0 International license.

FIGURE 6

a
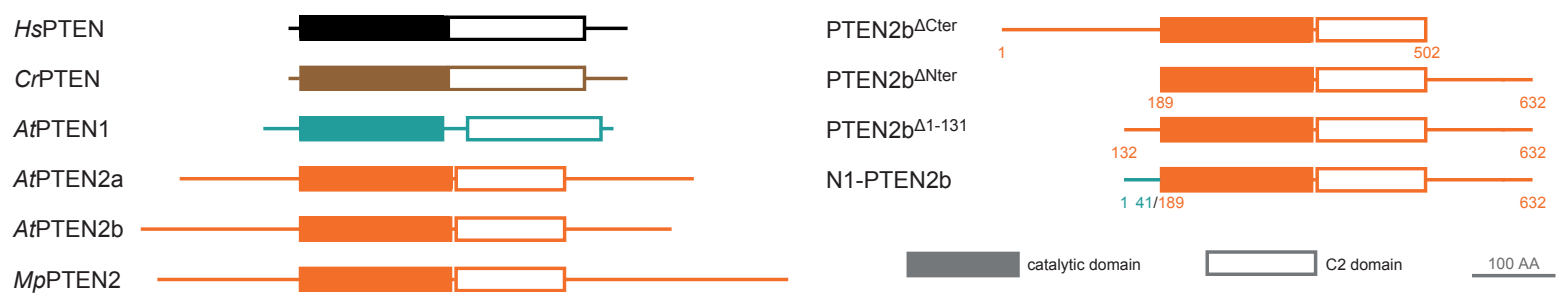

b
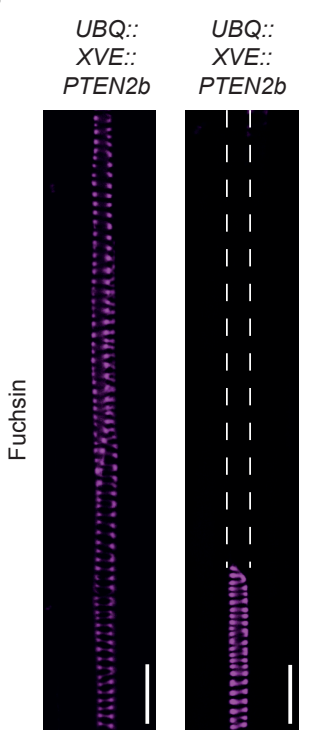

UBQ::
XVE.:

$U B Q::$

UBQ::

UBQ::

XVE:: XVE:: $\quad$ XVE::

C

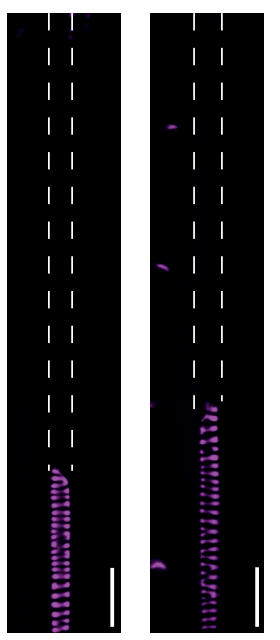
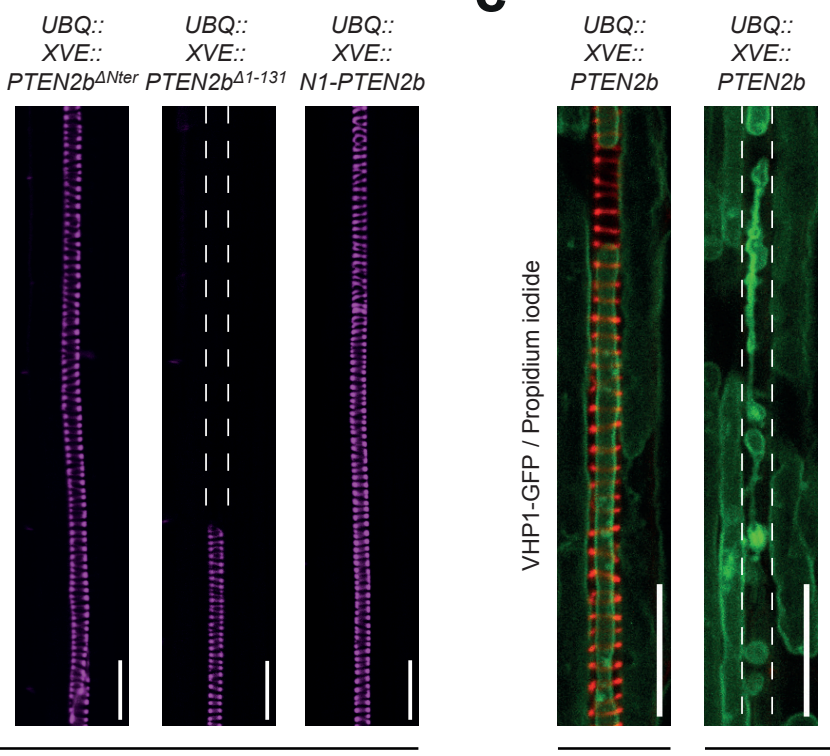

$U B Q::$
$X V E:$

UBQ::

PTEN2b

PTEN2bDCter PTEN2bDNter

mock

induced

mock

\begin{tabular}{llll}
1 & 1 \\
1 & 1 \\
1 & 1 \\
1 & 1 \\
1 & 1 \\
1 & 1 \\
1 & 1 \\
1 & 1 \\
1 & 1 \\
1 & 1 \\
1 & 1 \\
1 & 1 \\
1 & 1 \\
1 & 1 \\
1 & 1 \\
1 & 1 \\
1 & 1 \\
1 & 1 \\
\hline
\end{tabular}

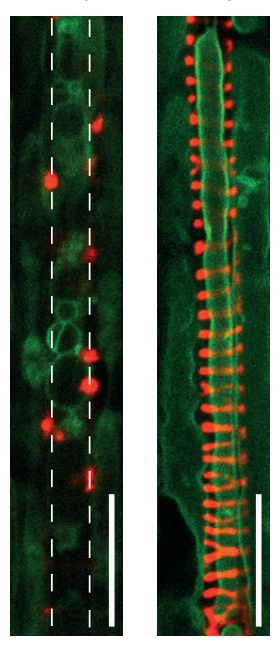

induced

d

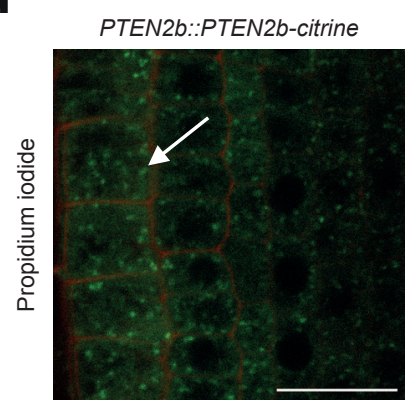

PTEN2b::PTEN2b ${ }^{\triangle N \text { ter }}$-citrine

PTEN2b::PTEN2b $b^{\Delta 1-131}$-citrine

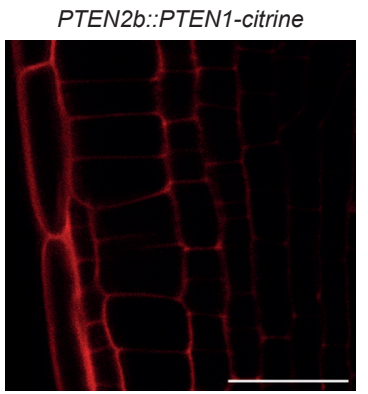

Figure 6: A conserved domain within PTEN2s $\mathrm{N}$-terminal sequence is critical for their functionality and TGN anchoring. a, Schematic representation of the PTEN enzymes from Homo Sapiens (Hs), Arabidopsis (At), Marchantia (Mp) and Chlamydomonas (Cr) analysed in this study. On the right are represented the truncated version of PTEN2b without the entire C- (PTEN2b $\left.{ }^{\Delta C t e r}\right)$ or N-terminal (PTEN2 $b^{\Delta N \text { ter }}$ ) sequences, PTEN2b with a partial N-terminal sequences $\left(P T E N 2 b^{\Delta 1-131}\right.$ ) and the hybrid version with PTEN1 N-terminal (N1-PTEN2b). Colour filled boxes represent phosphatase catalytic domains whereas empty squared boxes represent C2 domains. b, Representative confocal microscopy images of fuchsin-stained protoxylem strands after $2 \mu \mathrm{M}$ estradiol-mediated induction for $48 \mathrm{~h}$ of indicated PTEN2b versions. Protoxylem gap cells are highlighted with white dashed lines. c, Representative images of vacuole morphology in mature xylem cell upon $48 \mathrm{~h}$ overexpression of indicated PTEN2b variants. VHP1-GFP labels tonoplast, while propidium iodide labels cell wall. Protoxylem gap cells are highlighted with white dashed lines. d, Representative confocal images of 6-day-old plants harbouring indicated constructs illustrating the dependence of PTEN2b localization at TGN to its N-terminal. Scale bars represent $20 \mu \mathrm{m}$. 
bioRxiv preprint doi: https://doi.org/10.1101/2022.01.18.476728; this version posted January 20, 2022. The copyright holder for this preprint (which was not certified by peer review) is the author/funder, who has granted bioRxiv a license to display the preprint in perpetuity. It is made available under aCC-BY-NC-ND 4.0 International license.

\section{EXTENDED DATA FIGURE 6}

a

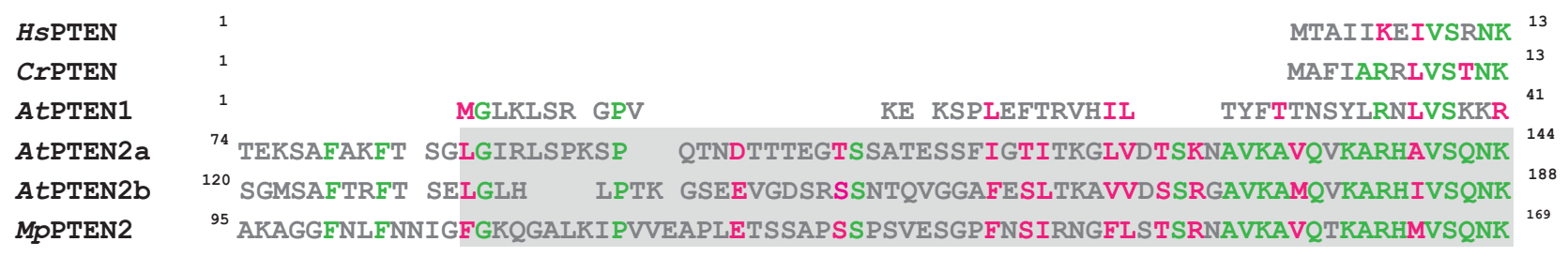

b
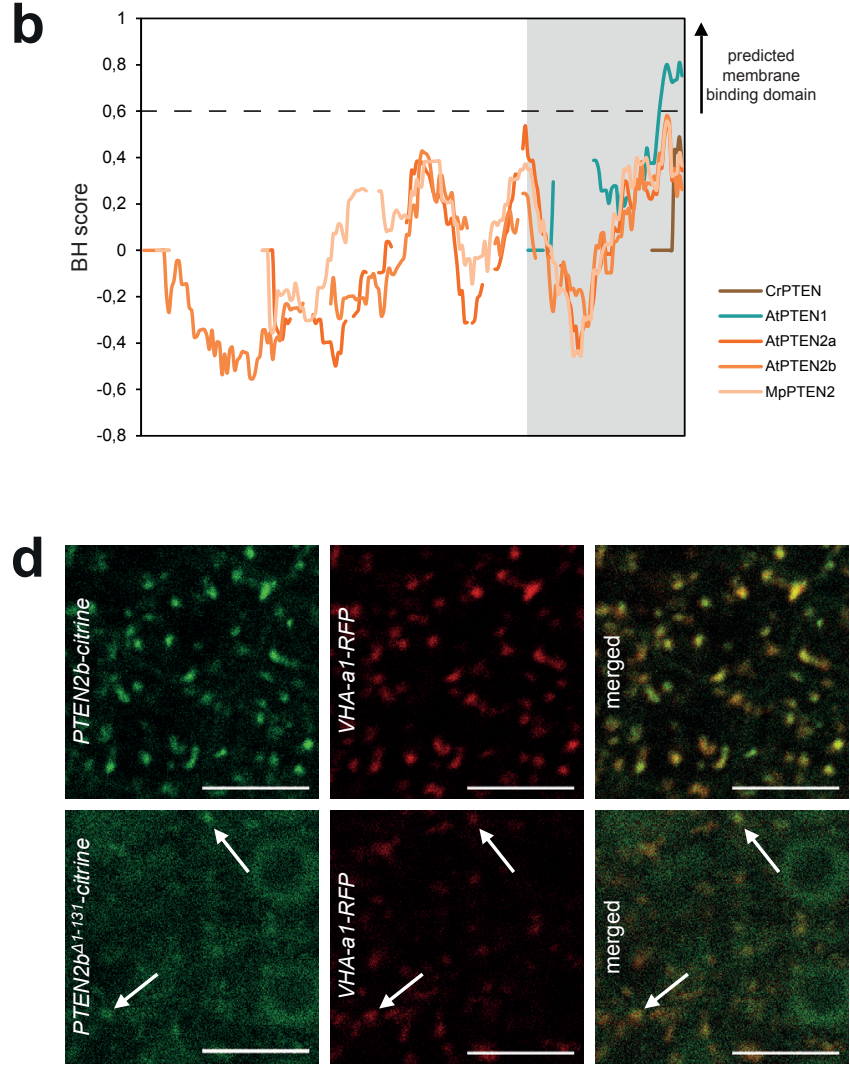
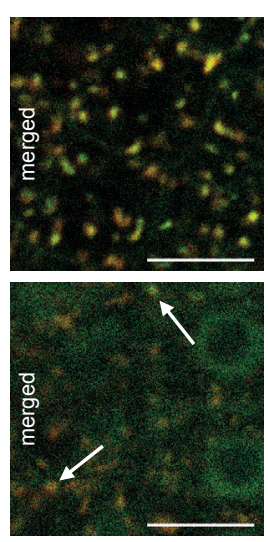

C

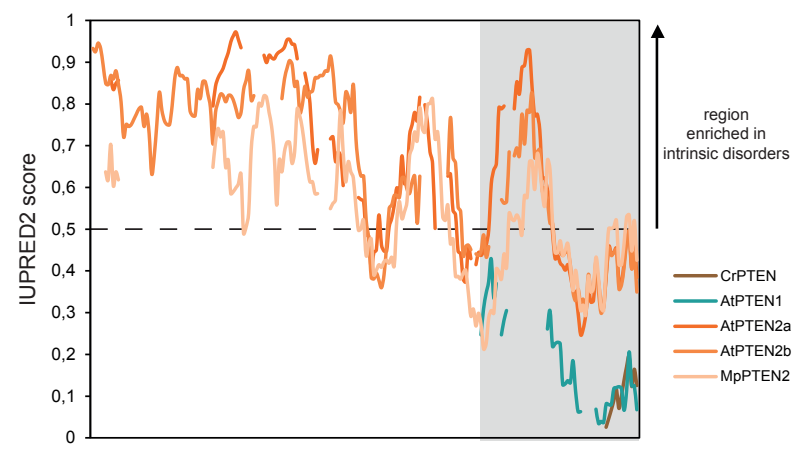

e
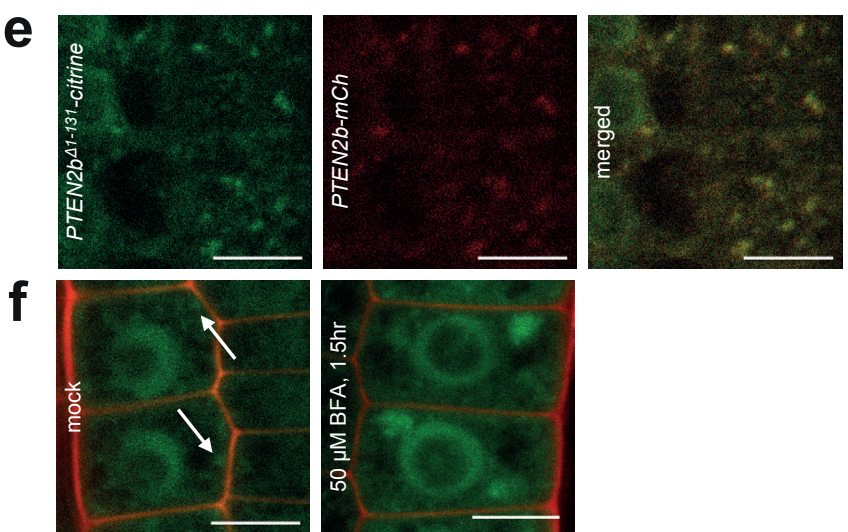

Extended Data Figure 6: The N-terminal domains of PTEN1 and PTEN2s exhibit different biochemical properties that determines their subcellular localization. a, Alignment of N-terminal PTEN sequences from: human (HsPTEN), Chlamydomonas (CrPTEN), Arabidopsis (AtPTEN1, AtPTEN2a and AtPTEN2b), and Marchantia (MpPTEN2) proteome assemblies obtained using CLUSTAL OMEGA. Identical amino acids are represented in green while similar amino acids are represented in magenta. $\mathbf{b}$, Prediction of membrane binding domain in PTEN N-terminal sequences of indicated isoform using $\mathrm{BH}$ score 74 . Domains with values above 0.6 are predicted to be membrane binding domain. $\mathbf{c}$, Prediction of intrinsic disordered region in $\mathrm{N}$-terminal sequences of indicated isoform using IUPred2A score ${ }^{75,76}$. Regions with values above 0.5 are supposed to be enriched in intrinsic disorders. The grey areas highlight the domain identified in PTEN2b (amino acid 132-188) as responsible for its functionality. d, Representative confocal images of 6 day-old plants expressing the TGN marker VHAa1-RFP together with PTEN2b::PTEN2b-CITRINE or PTEN2b::PTEN2b11-131-CITRINE. Arrows indicate the position of some of the dotted structures observed for PTEN2b::PTEN2b $b^{\Delta 1-131}$-CITRINE. e, Representative confocal images of 6 day-old plants expressing PTEN2b::PTEN2b $b^{11-131}-C I T R I N E$ with PTEN2b::PTEN2b-mCHERRY. Please note the co-localization of both constructs. f, Confocal images of 6 day-old plants expressing PTEN2b::PTEN2b $b^{11-131}-C I T R I N E$ and treated with DMSO or $50 \mu \mathrm{M}$ BFA for $1.5 \mathrm{~h}$. Scale bars represent $10 \mu \mathrm{m}$. 
bioRxiv preprint doi: https://doi.org/10.1101/2022.01.18.476728; this version posted January 20, 2022. The copyright holder for this preprint (which was not certified by peer review) is the author/funder, who has granted bioRxiv a license to display the preprint in perpetuity. It is made available under aCC-BY-NC-ND 4.0 International license.

FIGURE S1

a

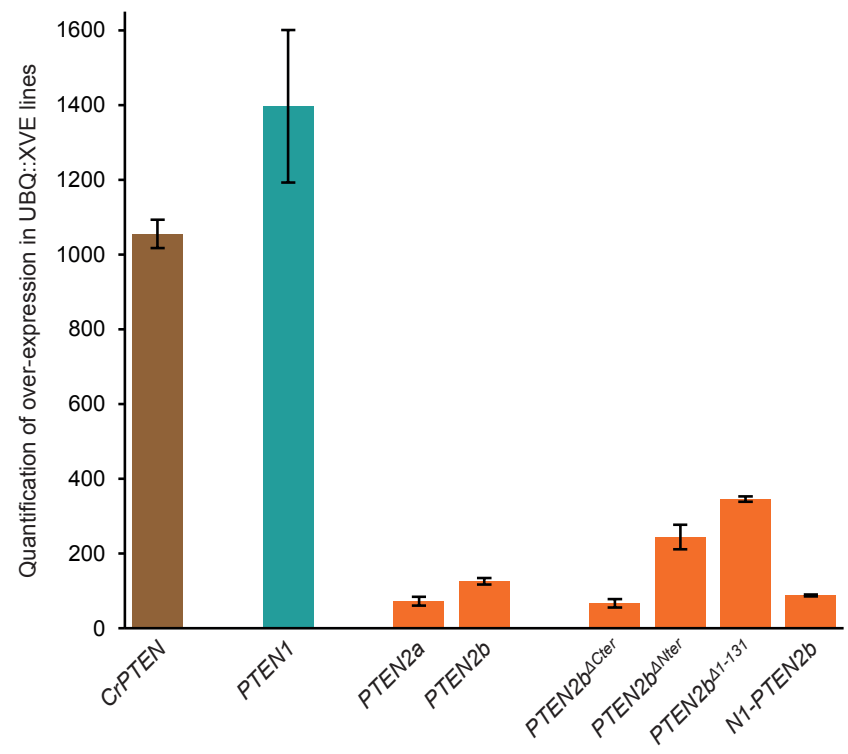

b

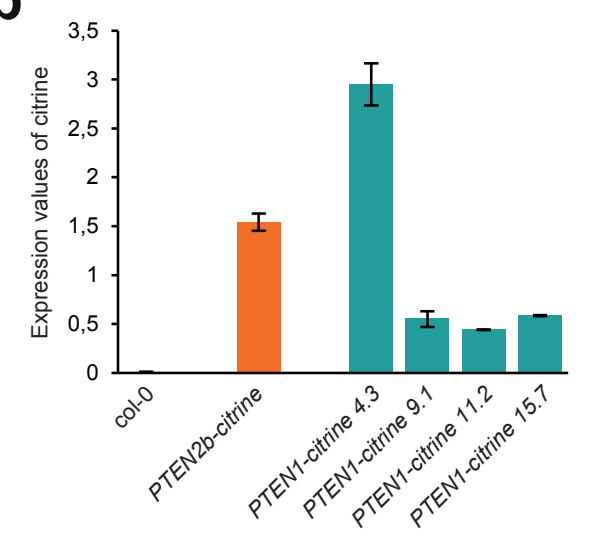

C $\mathrm{kDa}$

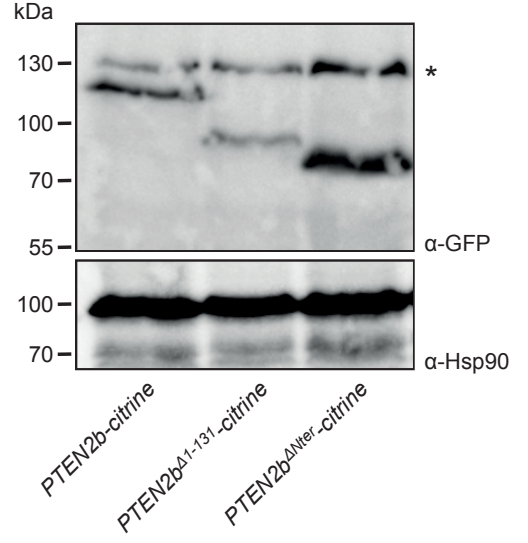

Supplementary Figure 1: Transgenic lines validation (Supporting data for Figures 6 and 7). a, qPCR analyses confirmed the over-expression of PTEN in different inducible lines described in Fig. 6 and Fig. 7. RNA was extracted from roots of 7 day-old plants treated with DMSO or $2 \mu \mathrm{M}$ estradiol for $48 \mathrm{hrs}$. Expression values of the different genes of interest in estradiol-treated plants were normalized by the corresponding expression values measured in DMSO-treated plants. Values represent the mean of two biological replicates (both including three technical replicates). Error bars indicate the standard deviation. b, qPCR analyses revealed the presence of CITRINE mRNA in independent PTEN2b::PTEN1-CITRINE that do not exhibit any fluorescence signal in root cells (Fig 6). Values represent the mean of three technical replicates, error bars indicate the standard deviation. c, The expression of the different Nter-truncated versions of PTEN2b tagged with citrine (Fig 6 ) was confirmed by Western blot using anti-GFP antibody. Anti-Hsp90 was used as a loading control. The star indicates the presence of an unspecific band. 
bioRxiv preprint doi: https://doi.org/10.1101/2022.01.18.476728; this version posted January 20, 2022. The copyright holder for this preprint (which was not certified by peer review) is the author/funder, who has granted bioRxiv a license to display the preprint in perpetuity. It is made available under aCC-BY-NC-ND 4.0 International license.

\begin{tabular}{|c|c|c|c|c|}
\hline \multicolumn{5}{|c|}{ Supplementary Table 1: Protein sequences used to build the phylogenetic tree } \\
\hline Organism & Protein name & Order & Clade & Database \\
\hline Acorus americanus & Acora.04G145300 & Acorales & Monocotyledones & phytozome \\
\hline Acorus americanus & Acora.11G167200 & Acorales & Monocotyledones & phytozome \\
\hline Aegilops tauschii & LOC109747383 & Poales & Monocotyledones & $\mathrm{NCBI}$ \\
\hline Alyssum linifolium & Alyli.0051s0214 & Brassicales & Eudicotyledones & phytozome \\
\hline Alyssum linifolium & Alyli.0020s0220 & Brassicales & Eudicotyledones & phytozome \\
\hline Alyssum linifolium & Alyli.0204s0005 & Brassicales & Eudicotyledones & phytozome \\
\hline Alyssum linifolium & Alyli.0031s0213 & Brassicales & Eudicotyledones & phytozome \\
\hline Alyssum linifolium & Alyli.0216s0006 & Brassicales & Eudicotyledones & phytozome \\
\hline Amaranthus hypochondriacus & $\mathrm{AH} 006150$ & Caryophyllales & Eudicotyledones & phytozome \\
\hline Amaranthus hypochondriacus & $\mathrm{AH} 001650$ & Caryophyllales & Eudicotyledones & phytozome \\
\hline Amborella trichopoda & $\begin{array}{l}\text { evm_27.model.AmTr_v1.0_scaff } \\
\text { old00177.28 }\end{array}$ & Amborellales & Amborellales & phytozome \\
\hline Amborella trichopoda & $\begin{array}{l}\text { evm_27.model.AmTr_v1.0_scaff } \\
\text { old00051.97 }\end{array}$ & Amborellales & Amborellales & phytozome \\
\hline Anacardium occidentale & Anaoc.0018s0955 & Sapindales & Eudicotyledones & phytozome \\
\hline Anacardium occidentale & Anaoc.0017s0442 & Sapindales & Eudicotyledones & phytozome \\
\hline Anacardium occidentale & Anaoc.1291s0006 & Sapindales & Eudicotyledones & phytozome \\
\hline Anacardium occidentale & Anaoc.0003s1165 & Sapindales & Eudicotyledones & phytozome \\
\hline Ananas comosus & Aco005843 & Poales & Monocotyledones & phytozome \\
\hline Ananas comosus & Aco024739 & Poales & Monocotyledones & phytozome \\
\hline Aquilegia coerulea & Aqcoe3G057800 & Ranunculales & Eudicotyledones & phytozome \\
\hline Aquilegia coerulea & Aqcoe5G142900 & Ranunculales & Eudicotyledones & phytozome \\
\hline Aquilegia coerulea & Aqcoe6G311500 & Ranunculales & Eudicotyledones & phytozome \\
\hline Arabidopsis halleri & Araha.12280s0002 & Brassicales & Eudicotyledones & phytozome \\
\hline Arabidopsis halleri & Araha.2717s0004 & Brassicales & Eudicotyledones & phytozome \\
\hline Arabidopsis lyrata & AL7G50930 & Brassicales & Eudicotyledones & phytozome \\
\hline Arabidopsis lyrata & AL3G32830 & Brassicales & Eudicotyledones & phytozome \\
\hline Arabidopsis lyrata & AL5G30230 & Brassicales & Eudicotyledones & phytozome \\
\hline Arabidopsis thaliana & At5g39400 & Brassicales & Eudicotyledones & phytozome \\
\hline Arabidopsis thaliana & At3g19420 & Brassicales & Eudicotyledones & phytozome \\
\hline Arabidopsis thaliana & At3g50110 & Brassicales & Eudicotyledones & phytozome \\
\hline Arachis hypogaea & $\begin{array}{c}\text { arahy.Tifrunner.gnm1.ann1.854 } \\
\text { N7M }\end{array}$ & Fabales & Eudicotyledones & phytozome \\
\hline Arachis hypogaea & $\begin{array}{l}\text { arahy.Tifrunner.gnm1.ann1.PFF } \\
\text { 9RU }\end{array}$ & Fabales & Eudicotyledones & phytozome \\
\hline Arachis hypogaea & $\begin{array}{c}\text { arahy.Tifrunner.gnm1.ann1.132 } \\
\text { VHE }\end{array}$ & Fabales & Eudicotyledones & phytozome \\
\hline Arachis hypogaea & $\begin{array}{c}\text { arahy.Tifrunner.gnm1.ann1.69L } \\
\text { NNJ }\end{array}$ & Fabales & Eudicotyledones & phytozome \\
\hline Arachis hypogaea & $\begin{array}{c}\text { arahy.Tifrunner.gnm1.ann1.AK4 } \\
\text { RCU }\end{array}$ & Fabales & Eudicotyledones & phytozome \\
\hline Asparagus officinalis & $\begin{array}{c}\text { evm.model.AsparagusV1_08.13 } \\
99\end{array}$ & Asparagales & Monocotyledones & phytozome \\
\hline Asparagus officinalis & $\underset{7}{\text { evm.model.AsparagusV1_07.85 }}$ & Asparagales & Monocotyledones & phytozome \\
\hline Asparagus officinalis & $\underset{54}{\text { evm.model.AsparagusV1_08.32 }}$ & Asparagales & Monocotyledones & phytozome \\
\hline Aureococcus anophagefferens & AURANDRAFT_26423 & Pelagomonadales & Heterokonts & NCBI \\
\hline Aureococcus anophagefferens & AURANDRAFT_71576 & Pelagomonadales & Heterokonts & $\mathrm{NCBI}$ \\
\hline Aureococcus anophagefferens & AURANDRAFT_70790 & Pelagomonadales & Heterokonts & $\mathrm{NCBI}$ \\
\hline Beta vulgaris & EL10Ac9g22309 & Caryophyllales & Eudicotyledones & phytozome \\
\hline Beta vulgaris & EL10Ac6g15699 & Caryophyllales & Eudicotyledones & phytozome \\
\hline
\end{tabular}


bioRxiv preprint doi: https://doi.org/10.1101/2022.01.18.476728; this version posted January 20, 2022. The copyright holder for this preprint (which was not certified by peer review) is the author/funder, who has granted bioRxiv a license to display the preprint in perpetuity. It is made available under aCC-BY-NC-ND 4.0 International license.

\begin{tabular}{|c|c|c|c|c|}
\hline Organism & Protein name & Order & Clade & Database \\
\hline Betula platyphylla & BPChr11G05686 & Fagales & Eudicotyledones & phytozome \\
\hline Betula platyphylla & BPChr06G22535 & Fagales & Eudicotyledones & phytozome \\
\hline Boechera stricta & Bostr.25849s0003 & Brassicales & Eudicotyledones & phytozome \\
\hline Boechera stricta & Bostr.19424s0720 & Brassicales & Eudicotyledones & phytozome \\
\hline Boechera stricta & Bostr.6864s0231 & Brassicales & Eudicotyledones & phytozome \\
\hline Brachypodium distachyon & Bradi4g08080 & Poales & Monocotyledones & phytozome \\
\hline Brachypodium hybridum & Brahy.D04G0113800 & Poales & Monocotyledones & phytozome \\
\hline Brachypodium hybridum & Brahy.S10G0102100 & Poales & Monocotyledones & phytozome \\
\hline Brachypodium sylvaticum & Brasy5G104500 & Poales & Monocotyledones & phytozome \\
\hline Brassica oleracea capitata & Bol040114 & Brassicales & Eudicotyledones & phytozome \\
\hline Brassica oleracea capitata & Bol018098 & Brassicales & Eudicotyledones & phytozome \\
\hline Brassica oleracea capitata & Bol031011 & Brassicales & Eudicotyledones & phytozome \\
\hline Brassica oleracea capitata & Bol016970 & Brassicales & Eudicotyledones & phytozome \\
\hline Brassica rapa & Brara.A02880 & Brassicales & Eudicotyledones & phytozome \\
\hline Brassica rapa & Brara.E02262 & Brassicales & Eudicotyledones & phytozome \\
\hline Brassica rapa & Brara.C04308 & Brassicales & Eudicotyledones & phytozome \\
\hline Cakile maritima & Camar.1507s0005 & Brassicales & Eudicotyledones & phytozome \\
\hline Cakile maritima & Camar.0166s0020 & Brassicales & Eudicotyledones & phytozome \\
\hline Cakile maritima & Camar.0140s0022 & Brassicales & Eudicotyledones & phytozome \\
\hline Cakile maritima & Camar.0046s0044 & Brassicales & Eudicotyledones & phytozome \\
\hline Capsella grandiflora & Cagra.12117s0008 & Brassicales & Eudicotyledones & phytozome \\
\hline Capsella grandiflora & Cagra.1211s0012 & Brassicales & Eudicotyledones & phytozome \\
\hline Capsella grandiflora & Cagra.26626s0001 & Brassicales & Eudicotyledones & phytozome \\
\hline Capsella rubella & Carub.0007s3654 & Brassicales & Eudicotyledones & phytozome \\
\hline Capsella rubella & Carub.0003s1923 & Brassicales & Eudicotyledones & phytozome \\
\hline Capsella rubella & Carub.0005s 1645 & Brassicales & Eudicotyledones & phytozome \\
\hline Carex littledalei & FCM35_KLT03997 & Cyperales & Monocotyledones & $\mathrm{NCBI}$ \\
\hline Carex littledalei & FCM35_KLT07398 & Cyperales & Monocotyledones & NCBI \\
\hline Carica papaya & evm.model.supercontig_14.140 & Brassicales & Eudicotyledones & phytozome \\
\hline Carica papaya & evm.model.supercontig_200.15 & Brassicales & Eudicotyledones & phytozome \\
\hline Carya illinoinensis & Caril.03G085200 & Juglandales & & phytozome \\
\hline Carya illinoinensis & Caril.04G059000 & Juglandales & & phytozome \\
\hline Carya illinoinensis & Caril.01G012500 & Juglandales & & phytozome \\
\hline Carya illinoinensis & Caril.06G171800 & Juglandales & & phytozome \\
\hline Carya illinoinensis & Caril.05G009200 & Juglandales & & phytozome \\
\hline Castanea dentata & Caden.01G186200 & Fagales & Eudicotyledones & phytozome \\
\hline Castanea dentata & Caden.02G186200 & Fagales & Eudicotyledones & phytozome \\
\hline Castanea dentata & Caden.07G119900 & Fagales & Eudicotyledones & phytozome \\
\hline Caulanthus amplexicaulis & Caamp.0044s0232 & Brassicales & Eudicotyledones & phytozome \\
\hline Caulanthus amplexicaulis & Caamp.1041s0897 & Brassicales & Eudicotyledones & phytozome \\
\hline Caulanthus amplexicaulis & Caamp.1039s1127 & Brassicales & Eudicotyledones & phytozome \\
\hline Caulanthus amplexicaulis & Caamp.0051s0388 & Brassicales & Eudicotyledones & phytozome \\
\hline Caulanthus amplexicaulis & Caamp.0078s0093 & Brassicales & Eudicotyledones & phytozome \\
\hline Caulanthus amplexicaulis & Caamp.0026s0499 & Brassicales & Eudicotyledones & phytozome \\
\hline Ceratodon purpureus & CepurGG1.8G115300 & Dicranales & Bryophytes & phytozome \\
\hline
\end{tabular}


bioRxiv preprint doi: https://doi.org/10.1101/2022.01.18.476728; this version posted January 20, 2022. The copyright holder for this preprint (which was not certified by peer review) is the author/funder, who has granted bioRxiv a license to display the preprint in perpetuity. It is made available under aCC-BY-NC-ND 4.0 International license.

\begin{tabular}{|c|c|c|c|c|}
\hline Organism & Protein name & Order & Clade & Database \\
\hline Ceratodon purpureus & CepurGG1.11G093000 & Dicranales & Bryophytes & phytozome \\
\hline Ceratopteris richardii & Ceric.25G072600 & Polypodiales & Monilophytes & phytozome \\
\hline Ceratopteris richardii & Ceric.16G021900 & Polypodiales & Monilophytes & phytozome \\
\hline Chara braunii & CBR_g29865 & Charales & Charophytes & NCBI \\
\hline Chara braunii & CBR_g30221 & Charales & Charophytes & $\mathrm{NCBI}$ \\
\hline Chenopodium quinoa & AUR62013248 & Caryophyllales & Eudicotyledones & phytozome \\
\hline Chenopodium quinoa & AUR62010267 & Caryophyllales & Eudicotyledones & phytozome \\
\hline Chlamydomonas reinhardtii & Cre06.g308400 & Chlamydomonales & Chlorophytes & phytozome \\
\hline Chromochloris zofigiensis & Cz03g22130.t1 & Sphaeropleales & Chlorophytes & phytozome \\
\hline Chrysochromulina tobinii & Ctob_005637 & Prymnesiales & Haptophytes & NCBI \\
\hline Chrysochromulina tobinii & Ctob_015421 & Prymnesiales & Haptophytes & NCBI \\
\hline Chrysochromulina tobinii & Ctob_003434 & Prymnesiales & Haptophytes & $\mathrm{NCBI}$ \\
\hline Chrysochromulina tobinii & Ctob_004562 & Prymnesiales & Haptophytes & $\mathrm{NCBI}$ \\
\hline Chrysochromulina tobinii & Ctob_005565 & Prymnesiales & Haptophytes & $\mathrm{NCBI}$ \\
\hline Cicer arietinum & Ca_02178 & Fabales & Eudicotyledones & phytozome \\
\hline Cicer arietinum & Ca_05590 & Fabales & Eudicotyledones & phytozome \\
\hline Cinnamomum kanehirae & CKAN_02766500 & Laurales & Magnoliides & phytozome \\
\hline Cinnamomum kanehirae & CKAN_02500000 & Laurales & Magnoliides & phytozome \\
\hline Cinnamomum kanehirae & CKAN_01173600 & Laurales & Magnoliides & phytozome \\
\hline Cinnamomum kanehirae & CKAN_00608500 & Laurales & Magnoliides & phytozome \\
\hline Citrus clementina & Ciclev10011769m & Sapindales & Eudicotyledones & phytozome \\
\hline Citrus clementina & Ciclev10025539m & Sapindales & Eudicotyledones & phytozome \\
\hline Citrus clementina & Ciclev10028042m & Sapindales & Eudicotyledones & phytozome \\
\hline Citrus sinensis & orange1.1g012952m & Sapindales & Eudicotyledones & phytozome \\
\hline Citrus sinensis & orange1.1g014325m & Sapindales & Eudicotyledones & phytozome \\
\hline Citrus sinensis & orange1.1g037030m & Sapindales & Eudicotyledones & phytozome \\
\hline Cleome violacea & Clevi.0004s2083 & Brassicales & Eudicotyledones & phytozome \\
\hline Cleome violacea & Clevi.0032s0574 & Brassicales & Eudicotyledones & phytozome \\
\hline Cleome violacea & Clevi.0005s 2503 & Brassicales & Eudicotyledones & phytozome \\
\hline Coccomyxa subellipsoidea & 66502 & & Chlorophytes & phytozome \\
\hline Coffea arabica & evm.model.Scaffold_952.463 & Gentianales & Eudicotyledones & phytozome \\
\hline Coffea arabica & evm.model.Scaffold_612.518 & Gentianales & Eudicotyledones & phytozome \\
\hline Coffea arabica & evm.model.Scaffold_634.624 & Gentianales & Eudicotyledones & phytozome \\
\hline Coffea arabica & evm.model.Scaffold_952.172 & Gentianales & Eudicotyledones & phytozome \\
\hline Corymbia citriodora & Cocit.A0156 & Myrtales & Eudicotyledones & phytozome \\
\hline Corymbia citriodora & Cocit.L5056 & Myrtales & Eudicotyledones & phytozome \\
\hline Corymbia citriodora & Cocit.G0778 & Myrtales & Eudicotyledones & phytozome \\
\hline Crambe hispanica & Crahi.1829s0005 & Brassicales & Eudicotyledones & phytozome \\
\hline Crambe hispanica & Crahi.0455s0005 & Brassicales & Eudicotyledones & phytozome \\
\hline Crambe hispanica & Crahi.0412s0031 & Brassicales & Eudicotyledones & phytozome \\
\hline Crambe hispanica & Crahi.0943s0003 & Brassicales & Eudicotyledones & phytozome \\
\hline Crambe hispanica & Crahi.0276s0014 & Brassicales & Eudicotyledones & phytozome \\
\hline Cucumis sativus & Cucsa.338540 & Cucurbitales & Eudicotyledones & phytozome \\
\hline Daucus carota & DCAR_001632 & Apiales & Eudicotyledones & phytozome \\
\hline Daucus carota & DCAR_029077 & Apiales & Eudicotyledones & phytozome \\
\hline
\end{tabular}


bioRxiv preprint doi: https://doi.org/10.1101/2022.01.18.476728; this version posted January 20, 2022. The copyright holder for this preprint (which was not certified by peer review) is the author/funder, who has granted bioRxiv a license to display the preprint in perpetuity. It is made available under aCC-BY-NC-ND 4.0 International license.

\begin{tabular}{|c|c|c|c|c|}
\hline Organism & Protein name & Order & Clade & Database \\
\hline Daucus carota & DCAR_010857 & Apiales & Eudicotyledones & phytozome \\
\hline Descurainia sophioides & Desop.0231s0613 & Brassicales & Eudicotyledones & phytozome \\
\hline Descurainia sophioides & Desop.0248s0666 & Brassicales & Eudicotyledones & phytozome \\
\hline Descurainia sophioides & Desop.0207s0242 & Brassicales & Eudicotyledones & phytozome \\
\hline Dichanthelium oligosanthes & BAE44_0008647 & Poales & Monocotyledones & $\mathrm{NCBI}$ \\
\hline Dioscorea alata & Dioal.19G152700 & Dioscoreales & Monocotyledones & phytozome \\
\hline Diptychocarpus strictus & Distr.0012s22625 & Brassicales & Eudicotyledones & phytozome \\
\hline Diptychocarpus strictus & Distr.0005s223300 & Brassicales & Eudicotyledones & phytozome \\
\hline Diptychocarpus strictus & Distr.0006s 170500 & Brassicales & Eudicotyledones & phytozome \\
\hline Ectocarpus siliculosus & Esi_0301_0020 & Ectocarpales & Heterokonts & $\mathrm{NCBI}$ \\
\hline Ectocarpus siliculosus & Esi_0147_0077 & Ectocarpales & Heterokonts & NCBI \\
\hline Ectocarpus siliculosus & Esi_0147_0079 & Ectocarpales & Heterokonts & $\mathrm{NCBI}$ \\
\hline Elaeis guineensis & LOC105056003 & Arecales & Monocotyledones & $\mathrm{NCBI}$ \\
\hline Elaeis guineensis & LOC105045483 & Arecales & Monocotyledones & $\mathrm{NCBI}$ \\
\hline Elaeis guineensis & LOC105035125 & Arecales & Monocotyledones & $\mathrm{NCBI}$ \\
\hline Eleusine coracana & ELECO.r07.5BG0436130 & Poales & Monocotyledones & phytozome \\
\hline Eleusine coracana & ELECO.r07.5AG0388310 & Poales & Monocotyledones & phytozome \\
\hline Emiliania huxleyi & EMIHUDRAFT_195717 & Isochrysidales & Haptophytes & NCBI \\
\hline Emiliania huxleyi & EMIHUDRAFT_437207 & Isochrysidales & Haptophytes & $\mathrm{NCBI}$ \\
\hline Eragrostis curvula & EJB05_06262 & Cyperales & Monocotyledones & $\mathrm{NCBI}$ \\
\hline Eragrostis curvula & EJB05_01954 & Cyperales & Monocotyledones & $\mathrm{NCBI}$ \\
\hline Eruca vesicaria & Eruve.3288s0001 & Brassicales & Eudicotyledones & phytozome \\
\hline Eruca vesicaria & Eruve.2621s0007 & Brassicales & Eudicotyledones & phytozome \\
\hline Eruca vesicaria & Eruve.2403s0005 & Brassicales & Eudicotyledones & phytozome \\
\hline Eruca vesicaria & Eruve.0429s0037 & Brassicales & Eudicotyledones & phytozome \\
\hline Eruca vesicaria & Eruve.1019s0020 & Brassicales & Eudicotyledones & phytozome \\
\hline Eruca vesicaria & Eruve.1393s0007 & Brassicales & Eudicotyledones & phytozome \\
\hline Eucalyptus grandis & Eucgr.J01367 & Myrtales & Eudicotyledones & phytozome \\
\hline Eucalyptus grandis & Eucgr.G01374 & Myrtales & Eudicotyledones & phytozome \\
\hline Eucalyptus grandis & Eucgr.100804 & Myrtales & Eudicotyledones & phytozome \\
\hline Euclidium syriacum & Eusyr.0002s0385 & Brassicales & Eudicotyledones & phytozome \\
\hline Euclidium syriacum & Eusyr.0017s0515 & Brassicales & Eudicotyledones & phytozome \\
\hline Euclidium syriacum & Eusyr.0120s0222 & Brassicales & Eudicotyledones & phytozome \\
\hline Eutrema salsugineum & Thhalv10027783m & Brassicales & Eudicotyledones & phytozome \\
\hline Eutrema salsugineum & Thhalv10020332m & Brassicales & Eudicotyledones & phytozome \\
\hline Eutrema salsugineum & Thhalv10010193m & Brassicales & Eudicotyledones & phytozome \\
\hline Fragaria vesca & FvH4_6g47530 & Rosales & Eudicotyledones & phytozome \\
\hline Fragaria vesca & FvH4_2g05490 & Rosales & Eudicotyledones & phytozome \\
\hline Fragaria vesca & FvH4_1g16580 & Rosales & Eudicotyledones & phytozome \\
\hline Ginkgo biloba & GBI00005494 & Ginkgoales & Acrogymnosperms & $\begin{array}{c}\text { Gymno } \\
\text { plaza } 1.0\end{array}$ \\
\hline Glycine max & GlysoPI483463.08G218300 & Fabales & Eudicotyledones & phytozome \\
\hline Glycine max & GlysoPI483463.01G151800 & Fabales & Eudicotyledones & phytozome \\
\hline Glycine max & GlysoPI483463.11G048000 & Fabales & Eudicotyledones & phytozome \\
\hline Glycine max & GlysoPI483463.10G224200 & Fabales & Eudicotyledones & phytozome \\
\hline Glycine max & GlysoPI483463.20G094900 & Fabales & Eudicotyledones & phytozome \\
\hline
\end{tabular}


bioRxiv preprint doi: https://doi.org/10.1101/2022.01.18.476728; this version posted January 20, 2022. The copyright holder for this preprint (which was not certified by peer review) is the author/funder, who has granted bioRxiv a license to display the preprint in perpetuity. It is made available under aCC-BY-NC-ND 4.0 International license.

\begin{tabular}{|c|c|c|c|c|}
\hline Organism & Protein name & Order & Clade & Database \\
\hline Gnetum montanum & GMO00031901 & Gnetales & Acrogymnosperms & $\begin{array}{c}\text { Gymno } \\
\text { plaza } 1.0\end{array}$ \\
\hline Gossypium bardadense & Gobar.A11G078400 & Malvales & Eudicotyledones & phytozome \\
\hline Gossypium bardadense & Gobar.D11G079100 & Malvales & Eudicotyledones & phytozome \\
\hline Gossypium bardadense & Gobar.D11G053600 & Malvales & Eudicotyledones & phytozome \\
\hline Gossypium bardadense & Gobar.A11G053700 & Malvales & Eudicotyledones & phytozome \\
\hline Gossypium bardadense & Gobar.D02G241200 & Malvales & Eudicotyledones & phytozome \\
\hline Gossypium bardadense & Gobar.A03G202000 & Malvales & Eudicotyledones & phytozome \\
\hline Gossypium bardadense & Gobar.A12G022900 & Malvales & Eudicotyledones & phytozome \\
\hline Gossypium bardadense & Gobar.D12G024700 & Malvales & Eudicotyledones & phytozome \\
\hline Gossypium bardadense & Gobar.D01G007700 & Malvales & Eudicotyledones & phytozome \\
\hline Gossypium bardadense & Gobar.A01G007300 & Malvales & Eudicotyledones & phytozome \\
\hline Gossypium hirsitum & Gohir.A11G071400 & Malvales & Eudicotyledones & phytozome \\
\hline Gossypium hirsitum & Gohir.D11G050950 & Malvales & Eudicotyledones & phytozome \\
\hline Gossypium hirsitum & Gohir.A11G047500 & Malvales & Eudicotyledones & phytozome \\
\hline Gossypium hirsitum & Gohir.D11G051100 & Malvales & Eudicotyledones & phytozome \\
\hline Guillardia theta & GUITHDRAFT_146552 & Pyrenomonadales & Cryptomonades & $\mathrm{NCBI}$ \\
\hline Guillardia theta & GUITHDRAFT_158972 & Pyrenomonadales & Cryptomonades & NCBI \\
\hline Guillardia theta & GUITHDRAFT_136489 & Pyrenomonadales & Cryptomonades & NCBI \\
\hline Helianthus annuus & HanXRQChr15g0473081 & Asterales & Eudicotyledones & phytozome \\
\hline Helianthus annuus & HanXRQChr03g0070181 & Asterales & Eudicotyledones & phytozome \\
\hline Helianthus annuus & HanXRQChr04g0128111 & Asterales & Eudicotyledones & phytozome \\
\hline Helianthus annuus & HanXRQChr11g0348401 & Asterales & Eudicotyledones & phytozome \\
\hline Hydrangea quercifolia & Hyque.05G148600 & Rosales & Eudicotyledones & phytozome \\
\hline Hydrangea quercifolia & Hyque.03G170500 & Rosales & Eudicotyledones & phytozome \\
\hline Hydrangea quercifolia & Hyque.13G030400 & Rosales & Eudicotyledones & phytozome \\
\hline Iberis amara & Ibeam.3529s0003 & Brassicales & Eudicotyledones & phytozome \\
\hline Iberis amara & Ibeam.1232s0005 & Brassicales & Eudicotyledones & phytozome \\
\hline Iberis amara & Ibeam.6756s0002 & Brassicales & Eudicotyledones & phytozome \\
\hline Iberis amara & Ibeam.3246s0002 & Brassicales & Eudicotyledones & phytozome \\
\hline Isatis tinctoria & Isati.8565s0004 & Brassicales & Eudicotyledones & phytozome \\
\hline Isatis tinctoria & Isati.0832s0027 & Brassicales & Eudicotyledones & phytozome \\
\hline Isatis tinctoria & Isati.0178s0011 & Brassicales & Eudicotyledones & phytozome \\
\hline Isatis tinctoria & Isati.1336s0016 & Brassicales & Eudicotyledones & phytozome \\
\hline Isatis tinctoria & Isati.1514s0004 & Brassicales & Eudicotyledones & phytozome \\
\hline Joinvillea ascendens & Joasc.14G102000 & Poales & Monocotyledones & phytozome \\
\hline Kalanchoe fedtschenkoi & Kaladp0026s0043 & Saxifragales & Eudicotyledones & phytozome \\
\hline Kalanchoe fedtschenkoi & Kaladp0015s0211 & Saxifragales & Eudicotyledones & phytozome \\
\hline Kalanchoe fedtschenkoi & Kaladp0058s0422 & Saxifragales & Eudicotyledones & phytozome \\
\hline Kalanchoe fedtschenkoi & Kaladp0028s0094 & Saxifragales & Eudicotyledones & phytozome \\
\hline Klebsormidium nitens & GAQ85806 & Klebsormidiales & Charophytes & $\mathrm{NCBI}$ \\
\hline Klebsormidium nitens & GAQ91864 & Klebsormidiales & Charophytes & $\mathrm{NCBI}$ \\
\hline Klebsormidium nitens & GAQ91212 & Klebsormidiales & Charophytes & $\mathrm{NCBI}$ \\
\hline Klebsormidium nitens & GAQ79654 & Klebsormidiales & Charophytes & $\mathrm{NCBI}$ \\
\hline Lactuca sativa & Lsat_1_v5_gn_3_98660 & Asterales & Eudicotyledones & phytozome \\
\hline Lactuca sativa & Lsat_1_v5_gn_3_98880 & Asterales & Eudicotyledones & phytozome \\
\hline
\end{tabular}


bioRxiv preprint doi: https://doi.org/10.1101/2022.01.18.476728; this version posted January 20, 2022. The copyright holder for this preprint (which was not certified by peer review) is the author/funder, who has granted bioRxiv a license to display the preprint in perpetuity. It is made available under aCC-BY-NC-ND 4.0 International license.

\begin{tabular}{|c|c|c|c|c|}
\hline Organism & Protein name & Order & Clade & Database \\
\hline Lactuca sativa & Lsat_1_v5_gn_7_89141 & Asterales & Eudicotyledones & phytozome \\
\hline Lactuca sativa & Lsat_1_v5_gn_3_98761 & Asterales & Eudicotyledones & phytozome \\
\hline Lactuca sativa & Lsat_1_v5_gn_3_98721 & Asterales & Eudicotyledones & phytozome \\
\hline Lactuca sativa & Lsat_1_v5_gn_3_90360 & Asterales & Eudicotyledones & phytozome \\
\hline Lactuca sativa & Lsat_1_v5_gn_9_111380 & Asterales & Eudicotyledones & phytozome \\
\hline Lactuca sativa & Lsat_1_v5_gn_5_70961 & Asterales & Eudicotyledones & phytozome \\
\hline Lepidium sativum & Lesat.0070s0837 & Brassicales & Eudicotyledones & phytozome \\
\hline Lepidium sativum & Lesat.0041s0230 & Brassicales & Eudicotyledones & phytozome \\
\hline Lepidium sativum & Lesat.0086s0034 & Brassicales & Eudicotyledones & phytozome \\
\hline Lepidium sativum & Lesat.0019s0255 & Brassicales & Eudicotyledones & phytozome \\
\hline Lepidium sativum & Lesat.0013s0306 & Brassicales & Eudicotyledones & phytozome \\
\hline Lepidium sativum & Lesat.0024s0016 & Brassicales & Eudicotyledones & phytozome \\
\hline Lindenbergia philippensis & Liphi.09G031000 & Lamiales & Eudicotyledones & phytozome \\
\hline Lindenbergia philippensis & Liphi.02G151000 & Lamiales & Eudicotyledones & phytozome \\
\hline Lindenbergia philippensis & Liphi.09G060300 & Lamiales & Eudicotyledones & phytozome \\
\hline Linum usitatissimum & Lus10027876 & Malpighiales & Eudicotyledones & phytozome \\
\hline Linum usitatissimum & Lus10002826 & Malpighiales & Eudicotyledones & phytozome \\
\hline Lotus japonicus & Lj1g0022114 & Fabales & Eudicotyledones & phytozome \\
\hline Lotus japonicus & Lj2g0026106 & Fabales & Eudicotyledones & phytozome \\
\hline Lotus japonicus & Lj5g0017669 & Fabales & Eudicotyledones & phytozome \\
\hline Lunaria annua & Luann.0281s0031 & Brassicales & Eudicotyledones & phytozome \\
\hline Lunaria annua & Luann.0007s0091 & Brassicales & Eudicotyledones & phytozome \\
\hline Lunaria annua & Luann.0026s0012 & Brassicales & Eudicotyledones & phytozome \\
\hline Lunaria annua & Luann.0006s0072 & Brassicales & Eudicotyledones & phytozome \\
\hline Lupinus albus & Lalb_Chr17g0337091 & Fabales & Eudicotyledones & phytozome \\
\hline Lupinus albus & Lalb_Chr04g0258551 & Fabales & Eudicotyledones & phytozome \\
\hline Lupinus albus & Lalb_Chr16g0378681 & Fabales & Eudicotyledones & phytozome \\
\hline Lupinus albus & Lalb_Chr21g0308431 & Fabales & Eudicotyledones & phytozome \\
\hline Malcomia maritima & Mamar.0082s0142 & Brassicales & Eudicotyledones & phytozome \\
\hline Malcomia maritima & Mamar.0029s0743 & Brassicales & Eudicotyledones & phytozome \\
\hline Malcomia maritima & Mamar.0003s0492 & Brassicales & Eudicotyledones & phytozome \\
\hline Malus domestica & MD17G1056300 & Rosales & Eudicotyledones & phytozome \\
\hline Malus domestica & MD09G1060900 & Rosales & Eudicotyledones & phytozome \\
\hline Malus domestica & MD02G1177900 & Rosales & Eudicotyledones & phytozome \\
\hline Malus domestica & MD15G1287800 & Rosales & Eudicotyledones & phytozome \\
\hline Malus domestica & MD05G1087100 & Rosales & Eudicotyledones & phytozome \\
\hline Manihot esculenta & Manes.02G050801 & Malpighiales & Eudicotyledones & phytozome \\
\hline Manihot esculenta & Manes.12G108000 & Malpighiales & Eudicotyledones & phytozome \\
\hline Manihot esculenta & Manes.10G077300 & Malpighiales & Eudicotyledones & phytozome \\
\hline Marchantia polymorpha & Mapoly0058s0093 & Marchantiales & Marchantiophytes & phytozome \\
\hline Marchantia polymorpha & Mapoly0016s0179 & Marchantiales & Marchantiophytes & phytozome \\
\hline Marchantia polymorpha & Mapoly0117s0012 & Marchantiales & Marchantiophytes & phytozome \\
\hline Medicago truncatula & Medtr7g012250 & Fabales & Eudicotyledones & phytozome \\
\hline Medicago truncatula & Medtr5g016550 & Fabales & Eudicotyledones & phytozome \\
\hline Medicago truncatula & Medtr1g107540 & Fabales & Eudicotyledones & phytozome \\
\hline
\end{tabular}


bioRxiv preprint doi: https://doi.org/10.1101/2022.01.18.476728; this version posted January 20, 2022. The copyright holder for this preprint (which was not certified by peer review) is the author/funder, who has granted bioRxiv a license to display the preprint in perpetuity. It is made available under aCC-BY-NC-ND 4.0 International license.

\begin{tabular}{|c|c|c|c|c|}
\hline Organism & Protein name & Order & Clade & Database \\
\hline Mimulus guttatus & Migut.H00656 & Lamiales & Eudicotyledones & phytozome \\
\hline Mimulus guttatus & Migut.H00402 & Lamiales & Eudicotyledones & phytozome \\
\hline Mimulus guttatus & Migut.B00375 & Lamiales & Eudicotyledones & phytozome \\
\hline Miscanthus sinensis & Misin03G029900 & Cyperales & Monocotyledones & phytozome \\
\hline Miscanthus sinensis & Misin04G012900 & Cyperales & Monocotyledones & phytozome \\
\hline Musa acuminata & GSMUA_Achr10P29220_001 & Zingiberales & Monocotyledones & phytozome \\
\hline Musa acuminata & GSMUA_Achr7P21640_001 & Zingiberales & Monocotyledones & phytozome \\
\hline Musa acuminata & GSMUA_Achr5P07740_001 & Zingiberales & Monocotyledones & phytozome \\
\hline Musa balbisiana & C4D60_Mb05t09990 & Zingiberales & Monocotyledones & $\mathrm{NCBI}$ \\
\hline Musa balbisiana & C4D60_Mb07t05750 & Zingiberales & Monocotyledones & NCBI \\
\hline Musa balbisiana & C4D60_Mb10t02290 & Zingiberales & Monocotyledones & $\mathrm{NCBI}$ \\
\hline Myagrum perfoliatum & Myper.0019s0558 & Brassicales & Eudicotyledones & phytozome \\
\hline Myagrum perfoliatum & Myper.0009s1500 & Brassicales & Eudicotyledones & phytozome \\
\hline Myagrum perfoliatum & Myper.0005s1433 & Brassicales & Eudicotyledones & phytozome \\
\hline Nelumbo nucifera & LOC104598017 & Proteales & Eudicotyledones & NCBI \\
\hline Nelumbo nucifera & LOC104589843 & Proteales & Eudicotyledones & $\mathrm{NCBI}$ \\
\hline Nelumbo nucifera & LOC104605416 & Proteales & Eudicotyledones & $\mathrm{NCBI}$ \\
\hline Nymphaea colorata & Nycol.I01022 & Nymphaeales & Nymphaeales & phytozome \\
\hline Nymphaea colorata & Nycol.C00833 & Nymphaeales & Nymphaeales & phytozome \\
\hline Olea europaea & Oeu062142 & Lamiales & Eudicotyledones & phytozome \\
\hline Olea europaea & Oeu014788 & Lamiales & Eudicotyledones & phytozome \\
\hline Olea europaea & Oeu001763 & Lamiales & Eudicotyledones & phytozome \\
\hline Olea europaea & Oeu044292 & Lamiales & Eudicotyledones & phytozome \\
\hline Oryza sativa & LOC_Os12g21890 & Poales & Monocotyledones & phytozome \\
\hline Panicum hallii & Pahal.2G380700 & Poales & Monocotyledones & phytozome \\
\hline Panicum virgatum & Pavir.2KG446900 & Poales & Monocotyledones & phytozome \\
\hline Panicum virgatum & Pavir.2NG500600 & Poales & Monocotyledones & phytozome \\
\hline Paspalum vaginatum & Pavag02G285600 & Poales & Monocotyledones & phytozome \\
\hline Pharus latifolius & Phala.10G061700 & Poales & Monocotyledones & phytozome \\
\hline Phaseolus vulgaris & Phvul.008G036300 & Fabales & Eudicotyledones & phytozome \\
\hline Phaseolus vulgaris & Phvul.002G015700 & Fabales & Eudicotyledones & phytozome \\
\hline Phaseolus vulgaris & Phvul.007G038300 & Fabales & Eudicotyledones & phytozome \\
\hline Phoenix dactylifera & LOC103711183 & Arecales & Monocotyledones & $\mathrm{NCBI}$ \\
\hline Phoenix dactylifera & LOC103706227 & Arecales & Monocotyledones & $\mathrm{NCBI}$ \\
\hline Phoenix dactylifera & LOC103705030 & Arecales & Monocotyledones & $\mathrm{NCBI}$ \\
\hline Physcomitrella patens & Pp3c19_18320V3 & Funariales & Bryophytes & phytozome \\
\hline Physcomitrella patens & Pp3c22_10340V3 & Funariales & Bryophytes & phytozome \\
\hline Physcomitrella patens & Pp3c21_8410V3 & Funariales & Bryophytes & phytozome \\
\hline Physcomitrella patens & Pp3c22_14420V3 & Funariales & Bryophytes & phytozome \\
\hline Picea glauca & PGL00015278 & Pinales & Acrogymnosperms & $\begin{array}{c}\text { Gymno } \\
\text { plaza } 1.0\end{array}$ \\
\hline Pinus pinaster & PPI00036745 & Pinales & Acrogymnosperms & $\begin{array}{l}\text { Gymno } \\
\text { plaza } 1.0\end{array}$ \\
\hline Pinus pinaster & PPI00005815 & Pinales & Acrogymnosperms & $\begin{array}{c}\text { Gymno } \\
\text { plaza } 1.0\end{array}$ \\
\hline Pinus pinaster & PPI00034805 & Pinales & Acrogymnosperms & $\begin{array}{l}\text { Gymno } \\
\text { plaza } 1.0\end{array}$ \\
\hline Pinus sylvestris & PSY00021791 & Pinales & Acrogymnosperms & $\begin{array}{c}\text { Gymno } \\
\text { plaza } 1.0\end{array}$ \\
\hline
\end{tabular}


bioRxiv preprint doi: https://doi.org/10.1101/2022.01.18.476728; this version posted January 20, 2022. The copyright holder for this preprint (which was not certified by peer review) is the author/funder, who has granted bioRxiv a license to display the preprint in perpetuity. It is made available under aCC-BY-NC-ND 4.0 International license.

\begin{tabular}{|c|c|c|c|c|}
\hline Organism & Protein name & Order & Clade & Database \\
\hline Pinus sylvestris & PSY00016716 & Pinales & Acrogymnosperms & $\begin{array}{l}\text { Gymno } \\
\text { plaza } 1.0\end{array}$ \\
\hline Pinus taeda & PTA00064026 & Pinales & Acrogymnosperms & $\begin{array}{l}\text { Gymno } \\
\text { plaza } 1.0\end{array}$ \\
\hline Pinus taeda & PTA00032593 & Pinales & Acrogymnosperms & $\begin{array}{l}\text { Gymno } \\
\text { plaza } 1.0\end{array}$ \\
\hline Pinus taeda & PTA00009584 & Pinales & Acrogymnosperms & $\begin{array}{c}\text { Gymno } \\
\text { plaza } 1.0\end{array}$ \\
\hline Poncirus trifoliata & Ptrif.0006s2220 & Sapindales & Eudicotyledones & phytozome \\
\hline Poncirus trifoliata & Ptrif.0007s0475 & Sapindales & Eudicotyledones & phytozome \\
\hline Poncirus trifoliata & Ptrif.0008s0442 & Sapindales & Eudicotyledones & phytozome \\
\hline Populus deltoides & Podel.17G093700 & Malpighiales & Eudicotyledones & phytozome \\
\hline Populus deltoides & Podel.07G056300 & Malpighiales & Eudicotyledones & phytozome \\
\hline Populus deltoides & Podel.01G321200 & Malpighiales & Eudicotyledones & phytozome \\
\hline Populus deltoides & Podel.09G100700 & Malpighiales & Eudicotyledones & phytozome \\
\hline Populus trichocarpa & Potri.017G089700 & Malpighiales & Eudicotyledones & phytozome \\
\hline Populus trichocarpa & Potri.007G047900 & Malpighiales & Eudicotyledones & phytozome \\
\hline Populus trichocarpa & Potri.001G302000 & Malpighiales & Eudicotyledones & phytozome \\
\hline Populus trichocarpa & Potri.009G098000 & Malpighiales & Eudicotyledones & phytozome \\
\hline Portulaca amilis & FUN_051737 & Caryophyllales & Eudicotyledones & phytozome \\
\hline Portulaca amilis & FUN_052803 & Caryophyllales & Eudicotyledones & phytozome \\
\hline Portulaca amilis & FUN_047201 & Caryophyllales & Eudicotyledones & phytozome \\
\hline Prunus persica & Prupe.3G259200 & Rosales & Eudicotyledones & phytozome \\
\hline Prunus persica & Prupe.6G230100 & Rosales & Eudicotyledones & phytozome \\
\hline Prunus persica & Prupe.8G131700 & Rosales & Eudicotyledones & phytozome \\
\hline Pseudotsuga menziesii & PME00026064 & Pinales & Acrogymnosperms & $\begin{array}{l}\text { Gymno } \\
\text { plaza } 1.0\end{array}$ \\
\hline Pseudotsuga menziesii & PME00001365 & Pinales & Acrogymnosperms & $\begin{array}{c}\text { Gymno } \\
\text { plaza } 1.0\end{array}$ \\
\hline Pseudotsuga menziesii & PME00077364 & Pinales & Acrogymnosperms & $\begin{array}{c}\text { Gymno } \\
\text { plaza } 1.0\end{array}$ \\
\hline Quercus rubra & Qurub.02G186400 & Fagales & Eudicotyledones & phytozome \\
\hline Quercus rubra & Qurub.11G098700 & Fagales & Eudicotyledones & phytozome \\
\hline Quercus rubra & Qurub.06G075200 & Fagales & Eudicotyledones & phytozome \\
\hline Ricinus communis & 29970.m000976 & Malpighiales & Eudicotyledones & phytozome \\
\hline Ricinus communis & 29801.m003123 & Malpighiales & Eudicotyledones & phytozome \\
\hline Rorippa islandica & Roisl.0070s0018 & Brassicales & Eudicotyledones & phytozome \\
\hline Rorippa islandica & Roisl.0046s0950 & Brassicales & Eudicotyledones & phytozome \\
\hline Salix purpurea & Sapur.017G073600 & Salicales & Eudicotyledones & phytozome \\
\hline Salix purpurea & Sapur.007G045200 & Salicales & Eudicotyledones & phytozome \\
\hline Salix purpurea & Sapur.016G181600 & Salicales & Eudicotyledones & phytozome \\
\hline Salix purpurea & Sapur.009G076600 & Salicales & Eudicotyledones & phytozome \\
\hline Schrenkiella parvula & Sp7g02580 & Brassicales & Eudicotyledones & phytozome \\
\hline Schrenkiella parvula & Sp3g17480 & Brassicales & Eudicotyledones & phytozome \\
\hline Schrenkiella parvula & Sp5g12010 & Brassicales & Eudicotyledones & phytozome \\
\hline Selaginella moellendorffii & 91219 & Selaginellales & Lycophytes & phytozome \\
\hline Selaginella moellendorffii & 165134 & Selaginellales & Lycophytes & phytozome \\
\hline Setaria italica & Seita.2G322700 & Cyperales & Monocotyledones & phytozome \\
\hline Setaria viridis & Sevir.2G334300 & Cyperales & Monocotyledones & phytozome \\
\hline Sinapis alba & Sialb.0606s 0023 & Brassicales & Eudicotyledones & phytozome \\
\hline
\end{tabular}


bioRxiv preprint doi: https://doi.org/10.1101/2022.01.18.476728; this version posted January 20, 2022. The copyright holder for this preprint (which was not certified by peer review) is the author/funder, who has granted bioRxiv a license to display the preprint in perpetuity. It is made available under aCC-BY-NC-ND 4.0 International license.

\begin{tabular}{|c|c|c|c|c|}
\hline Organism & Protein name & Order & Clade & Database \\
\hline Sinapis alba & Sialb.0540s0029 & Brassicales & Eudicotyledones & phytozome \\
\hline Sinapis alba & Sialb.0766s0022 & Brassicales & Eudicotyledones & phytozome \\
\hline Sinapis alba & Sialb.0054s0274 & Brassicales & Eudicotyledones & phytozome \\
\hline Sinapis alba & Sialb.0001s0595 & Brassicales & Eudicotyledones & phytozome \\
\hline Sinapis alba & Sialb.0672s 0053 & Brassicales & Eudicotyledones & phytozome \\
\hline Solanum lycopersicum & Solyc03g013310 & Solanales & Eudicotyledones & phytozome \\
\hline Solanum lycopersicum & Solyc01g107750 & Solanales & Eudicotyledones & phytozome \\
\hline Solanum lycopersicum & Solyc02g093000 & Solanales & Eudicotyledones & phytozome \\
\hline Solanum tuberosum & Soltu.DM.02G028260 & Solanales & Eudicotyledones & phytozome \\
\hline Solanum tuberosum & Soltu.DM.03G007160 & Solanales & Eudicotyledones & phytozome \\
\hline Solanum tuberosum & Soltu.DM.01G047110 & Solanales & Eudicotyledones & phytozome \\
\hline Sorghum bicolor & Sobic.002G008800 & Poales & Monocotyledones & phytozome \\
\hline Sphagnum fallax & Sphfalx16G057000 & Sphagnales & Bryophytes & phytozome \\
\hline Sphagnum fallax & Sphfalx17G006600 & Sphagnales & Bryophytes & phytozome \\
\hline Sphagnum fallax & Sphfalx18G079700 & Sphagnales & Bryophytes & phytozome \\
\hline Sphagnum fallax & Sphfalx13G023500 & Sphagnales & Bryophytes & phytozome \\
\hline Sphagnum fallax & Sphfalx14G024100 & Sphagnales & Bryophytes & phytozome \\
\hline Sphagnum fallax & Sphfalx06G107500 & Sphagnales & Bryophytes & phytozome \\
\hline Sphagnum magellanicum & Sphmag16G056400 & Sphagnages & Bryophytes & phytozome \\
\hline Sphagnum magellanicum & Sphmag17G006400 & Sphagnages & Bryophytes & phytozome \\
\hline Sphagnum magellanicum & Sphmag06G111300 & Sphagnages & Bryophytes & phytozome \\
\hline Sphagnum magellanicum & Sphmag18G019000 & Sphagnages & Bryophytes & phytozome \\
\hline Sphagnum magellanicum & Sphmag13G020000 & Sphagnages & Bryophytes & phytozome \\
\hline Sphagnum magellanicum & Sphmag14G023700 & Sphagnages & Bryophytes & phytozome \\
\hline Spinacia oleracea & Spov3_C0007.00101 & Caryophyllales & Eudicotyledones & phytozome \\
\hline Spinacia oleracea & Spov3_chr1.02493 & Caryophyllales & Eudicotyledones & phytozome \\
\hline Spirodela polyrhiza & Spipo2G0093300 & Alismatales & Monocotyledones & phytozome \\
\hline Spirodela polyrhiza & Spipo4G0005500 & Alismatales & Monocotyledones & phytozome \\
\hline Stanleya pinnata & Stapi.1453s0002 & Brassicales & Eudicotyledones & phytozome \\
\hline Stanleya pinnata & Stapi.1874s0008 & Brassicales & Eudicotyledones & phytozome \\
\hline Stanleya pinnata & Stapi.1161s0006 & Brassicales & Eudicotyledones & phytozome \\
\hline Stanleya pinnata & Stapi.0737s0004 & Brassicales & Eudicotyledones & phytozome \\
\hline Stanleya pinnata & Stapi.0692s0011 & Brassicales & Eudicotyledones & phytozome \\
\hline Taxus baccata & TBA00001628 & Taxales & Acrogymnosperms & $\begin{array}{c}\text { Gymno } \\
\text { plaza } 1.0\end{array}$ \\
\hline Taxus baccata & TBA00011682 & Taxales & Acrogymnosperms & $\begin{array}{c}\text { Gymno } \\
\text { plaza } 1.0\end{array}$ \\
\hline Theobroma cacao & Thecc.04G090400 & Malvales & Eudicotyledones & phytozome \\
\hline Theobroma cacao & Thecc.01G062500 & Malvales & Eudicotyledones & phytozome \\
\hline Theobroma cacao & Thecc.02G105700 & Malvales & Eudicotyledones & phytozome \\
\hline Thinopyrum intermedium & Thint.15G0279400 & Poales & Monocotyledones & phytozome \\
\hline Thinopyrum intermedium & Thint.13G0199200 & Poales & Monocotyledones & phytozome \\
\hline Thinopyrum intermedium & Thint.14G0237600 & Poales & Monocotyledones & phytozome \\
\hline Thlaspi arvense & Thlar.0083s0010 & Brassicales & Eudicotyledones & phytozome \\
\hline Thlaspi arvense & Thlar.0014s 0526 & Brassicales & Eudicotyledones & phytozome \\
\hline Thlaspi arvense & Thlar.0021s1378 & Brassicales & Eudicotyledones & phytozome \\
\hline Thuja plicata & Thupl.29382416s0045 & Pinales & Acrogymnosperms & phytozome \\
\hline
\end{tabular}


bioRxiv preprint doi: https://doi.org/10.1101/2022.01.18.476728; this version posted January 20, 2022. The copyright holder for this preprint (which was not certified by peer review) is the author/funder, who has granted bioRxiv a license to display the preprint in perpetuity. It is made available under aCC-BY-NC-ND 4.0 International license.

\begin{tabular}{|ccccc|}
\hline Organism & Protein name & Order & Clade & Database \\
Thuja plicata & Thupl.29377609s0023 & Pinales & Acrogymnosperms & phytozome \\
Trifolium pratense & Tp57577_TGAC_v2_mRNA345 & Fabales & Eudicotyledones & phytozome \\
Trifolium pratense & Tp57577_TGAC_v2_mRNA278 & Fabales & Eudicotyledones & phytozome \\
Trifolium pratense & Tp57577_TGAC_v2_mRNA143 & Fabales & Eudicotyledones & phytozome \\
Urochloa fusca & Urofu.1G032300 & Poales & Monocotyledones & phytozome \\
Urochloa fusca & Urofu.2G334900 & Poales & Monocotyledones & phytozome \\
Vigna unguiculata & Vigun05g037300 & Fabales & Eudicotyledones & phytozome \\
Vigna unguiculata & Vigun07g259200 & Fabales & Eudicotyledones & phytozome \\
Vigna unguiculata & Vigun02g146800 & Fabales & Eudicotyledones & phytozome \\
Vitis vinifera & VIT_214s0108g01410 & Vitales & Eudicotyledones & phytozome \\
Vitis vinifera & VIT_203s0180g00020 & Vitales & Eudicotyledones & phytozome \\
Vitis vinifera & VIT_207s0031g00020 & Vitales & Eudicotyledones & phytozome \\
Volvox carteri & Vocar.0030s0065 & Chlamydomonadal & Chlorophytes & phytozome \\
Zea mays & Zm00001d007942 & Cyperales & Monocotyledones & phytozome \\
Zostera marina & Zosma06g28190 & Alismatales & Monocotyledones & phytozome \\
Zostera marina & Zosma01g09840 & Alismatales & Monocotyledones & phytozome \\
\hline
\end{tabular}




\begin{tabular}{|c|c|c|}
\hline \multicolumn{3}{|c|}{ Supplementary Table 2: Primers used in this study } \\
\hline pVND7_FW_attB4 & 5'- GGGGACAACTTTGTATAGAAAAGTTGTCCTGCCGGTAAAGTGGAGAAG -3' & VND7 promoter \\
\hline pVND7_RV_attB1r & 5’- GGGGACTGCTTTTTTTGTACAAACTTGTCCACGATGATCCTATAAACG -3' & VND7 promoter \\
\hline pXCP1_FW_attB4 & 5'- GGGGACAACTTTGTATAGAAAAGTTGTCGCATTGCTGTGTCGATGG -3' & XCP1 promoter \\
\hline pXCP1_RV_attB1r & 5’- GGGGACTGCTTTTTTTGTACAAACTTGTAGCCAAATTTGTTCACTG -3' & XCP1 promoter \\
\hline XCP1_FW_attB1 & 5'- GGGGACAAGTTTGTACAAAAAAGCAGGCTTCATGGCTTTTTCTGCACCATCAC -3' & XCP1 coding \\
\hline XCP1_NS_attB2 & 5'- GGGGACCACTTTGTACAAGAAAGCTGGGTACTTGGTCTTGGTAGGATATG -3' & $X C P 1$ coding \\
\hline pBFN1-1975_FW_attB4 & 5'- GGGGACAACTTTGTATAGAAAAGTTGGAAATTAAGTATTTACCTGCCAAAAG -3' & BFN1 promoter \\
\hline pBFN1_RV_attB1r & 5'- GGGGACTGCTTTTTTTGTACAAACTTGATCTTCAAAGTTTGAAACTTATATAATG -3' & BFN1 promoter \\
\hline pCESA4_FW_attB4 & 5’- GGGGACAACTTTGTATAGAAAAGTTGGACATGCGATGGCATGGATGC -3' & CESA4 promoter \\
\hline pCESA4_RV_attB1r & 5'- GGGGACTGCTTTTTTTGTACAAACTTGGGCGAGGTACACTGAGCTCTC -3' & CESA4 promoter \\
\hline pCESA7_FW_attB4 & 5'- GGGGACAACTTTGTATAGAAAAGTTGCCCAGTTTGGAACGACACTTAGAAAAATAAG -3' & CESA7 promoter \\
\hline pCESA7_RV_attB1r & 5'- GGGGACTGCTTTTTTTGTACAAACTTGGAGGGACGGCCGGAGATTAG -3' & CESA7 promoter \\
\hline pCESA8_FW_attB4 & 5'- GGGGACAACTTTGTATAGAAAAGTTGCGCCTCACAATGTGTTCTTGC -3' & CESA8 promoter \\
\hline pCESA8_RV_attB1r & 5'- GGGGACTGCTTTTTTGTACAAACTTGCTTCGAATTCCCCTGTTTGGAG -3' & CESA8 promoter \\
\hline pPTEN2a_FW_attB4 & 5'- GGGGACAACTTTGTATAGAAAAGTTGTGAATAAACATGTAATCTCCATTTTTTTGTTCTC -3' & PTEN2a promoter \\
\hline pPTEN2a_RV_attB1r & 5’- GGGGACTGCTTTTTTTGTACAAACTTGCGTTTCTATCTTAATCCAAAATGTGAATTCTC -3' & PTEN2a promoter \\
\hline pPTEN2b_FW_attB4 & 5’- GGGGACAACTTTGTATAGAAAAGTTGCTAGATTTTAACTTGTGGTATACCGC -3' & PTEN2b promoter \\
\hline pPTEN2b_RV_attB1r & 5’- GGGGACTGCTTTTTTTGTACAAACTTGTTTAGCAATCCAACGCTAGCTC -3' & PTEN2b promoter \\
\hline PTEN1_FW_attB1 & 5'- GGGGACAAGTTTGTACAAAAAAGCAGGCTCC ATGGGTCTCAAGCTCTCACGAG -3' & PTEN1 coding \\
\hline PTEN1_NS_RV_attB2 & 5'- GGGGACCACTTTGTACAAGAAAGCTGGGTCAGAGAGAGAAAGGTCATCGCGG -3' & PTEN1 coding \\
\hline PTEN1_RV_attB2 & 5'- GGGGACCACTTTGTACAAGAAAGCTGGGTCTCAAGAGAGAGAAAGGTCATCGCG - 3' & PTEN1stop coding \\
\hline PTEN2a_FW_BstBI & 5'- ATTTTCGAATGTCGTCTGAGTCACCGAATTTG -3' & PTEN2a coding \\
\hline PTEN2a_NS_RV_Spel & 5'- ATTACTAGTATCGCTTTCAAAGTCGTCTTCATCTC -3' & PTEN2a coding \\
\hline PTEN2a_RV_Spēl & 5'- ATTACTAGTTCAATCGCTTTCAAAGTCGTCTTCATC -3' & PTEN2astop coding \\
\hline PTEN2b_FW_attB1 & 5'- GGGGACAAGTTTGTACAAAAAAGCAGGCTCCATGGAAACTGATCCTGCTAACTCTTC -3' & PTEN2b coding \\
\hline PTEN2b_NS_RV_attB2 & 5'- GGGGACCACTTTGTACAAGAAAGCTGGGTCGTCGCTTTCATAGTCTTCTTCCTC -3' & PTEN2b coding \\
\hline PTEN2b_RV_attB̄2 & 5'- GGGGACCACTTTGTACAAGAAAGCTGGGTCTCAGTCGCTTTCATAGTCTTCTTCC -3' & PTEN2bstop coding \\
\hline PTEN2b_Nter_FW_attB1 & 5'- GGGGACAAGTTTGTACAAAAAAGCAGGCTCCATGAGAAGATACCAGGTATGG -3' & PTEN2b $\triangle$ Nter gDNA \\
\hline PTEN2b_Nter_FW_attB1 & 5'- GGGGACAAGTTTGTACAAAAAAGCAGGCTCCATGAGAAGATACCAGGAGGGGG -3' & PTEN2b $\triangle N$ ter cDNA \\
\hline PTEN2bACter_RV_attB2 & 5'- GGGGACCACTTTGTACAAGAAAGCTGGGTCTCAGGGTTCTATCATTACGATCTCG -3' & PTEN2b $\Delta$ Cter coding \\
\hline PTEN2bD1-131_FW_attB1 & 5'- GGGGACAAGTTTGTACAAAAAAGCAGGCTCCATGCTTGGTCTGCATTTGCCAACG -3' & PTEN2b_1-131 coding \\
\hline PTEN1 ${ }^{\text {Nterm }} R_{R} V^{-}$ & 5'- GGTATCTTCTTCGTTTCTTGGACACCAAGTTACG -3' & PTEN1 Nter codding \\
\hline PTEN2b-Nter_FW & 5'- CCAAGAAACGAATGAGAAGATACCAGGTATGG -3' & PTEN2b-Nter gDNA \\
\hline
\end{tabular}




\begin{tabular}{|c|c|c|}
\hline \multicolumn{3}{|c|}{ Supplementary Table 2: Primers used in this study (continuation) } \\
\hline Primer Name & Primer Sequence & Objective \\
\hline PTEN2b-Nter_FW & 5'- CCAAGAAACGAAGAAGATACCAGGAGGGGG -3' & PTEN2b-Nter cDNA \\
\hline MpPTEN2 attB1_FW & 5'- GGGGACAAGTTTGTACAAAAAAGCAGGCTCCATGGACGACTCAGCCAACAG -3' & MpPTEN2 coding \\
\hline MpPTEN2_attB1_RV & 5'- GGGGACCACTTTGTACAAGAAAGCTGGGTCTCAATCTTCATCACTCTCGAAATCC -3' & MpPTEN2 coding \\
\hline qPTEN1_FW & 5'- GTCCGTGCTCGATATGCGACATC -3' & qPCR PTEN1 \\
\hline qPTEN1_RV & 5'- GTACGCCGATACCATTAGCCCTG -3' & qPCR PTEN1 \\
\hline qPTEN2a_FW & 5'-CAACCAGGCCGTAGGTGTATGC-3' & qPCR PTEN2a \\
\hline qPTEN2a_RV & 5'-CCCCTTTCTTTGGGGCACTGAAC-3' & qPCR PTEN2a \\
\hline qPTEN2b_FW & 5'-GCTGAAGAGGCTATTGATTACT-3' & qPCR PTEN2b \\
\hline qPTEN2b_RV & 5'-AAATCCTCTGAGCATGCATCTTCGC-3' & qPCR PTEN2b \\
\hline qCRPTEN FW & 5'AGAGGTGGTGGGAAAGATG-3' & qPCR CrPTEN \\
\hline qCrPTEN RV & 5'-GAGGTTGGAAATAAGGAAGAGG-3' & qPCR CrPTEN \\
\hline qCITRINE FW & 5'-GAAGTTCATCTGCACCACC-3' & qPCR CITRINE \\
\hline qCITRINE RV & 5'-TTGTACTCCAGCTTGTGCC-3' & qPCR CITRINE \\
\hline qPDF2 FW & 5'- TAACGTGGCCAAAATGATGC-3' & qPCR PDF2 \\
\hline qPDF2 RV & 5'-GTTCTCCACAACCGCTTGGT -3' & qPCR PDF2 \\
\hline pten2a_LP & 5'-CGGCAATATGTCATTATGCAG-3' & genotyping pten2a \\
\hline pten2a RP & 5'-TTTTTCCTGATCTGAATTCGAG-3' & genotyping pten $2 a$ \\
\hline pten2b_LP & 5'-CTCGAAAAATCCGAAAAGACC-3' & genotyping pten $2 b$ \\
\hline pten2b_RP & 5'-GAAGCGAATTTAGCCAAAACC-3' & genotyping pten $2 b$ \\
\hline cesa6_LP & 5'-GCTTGCAGCTGAATCAATACC-3' & genotyping cesa 6 \\
\hline cesa6 RP & 5'-AАCСTGATCAAATCCAATCCC-3' & genotyping cesa 6 \\
\hline
\end{tabular}




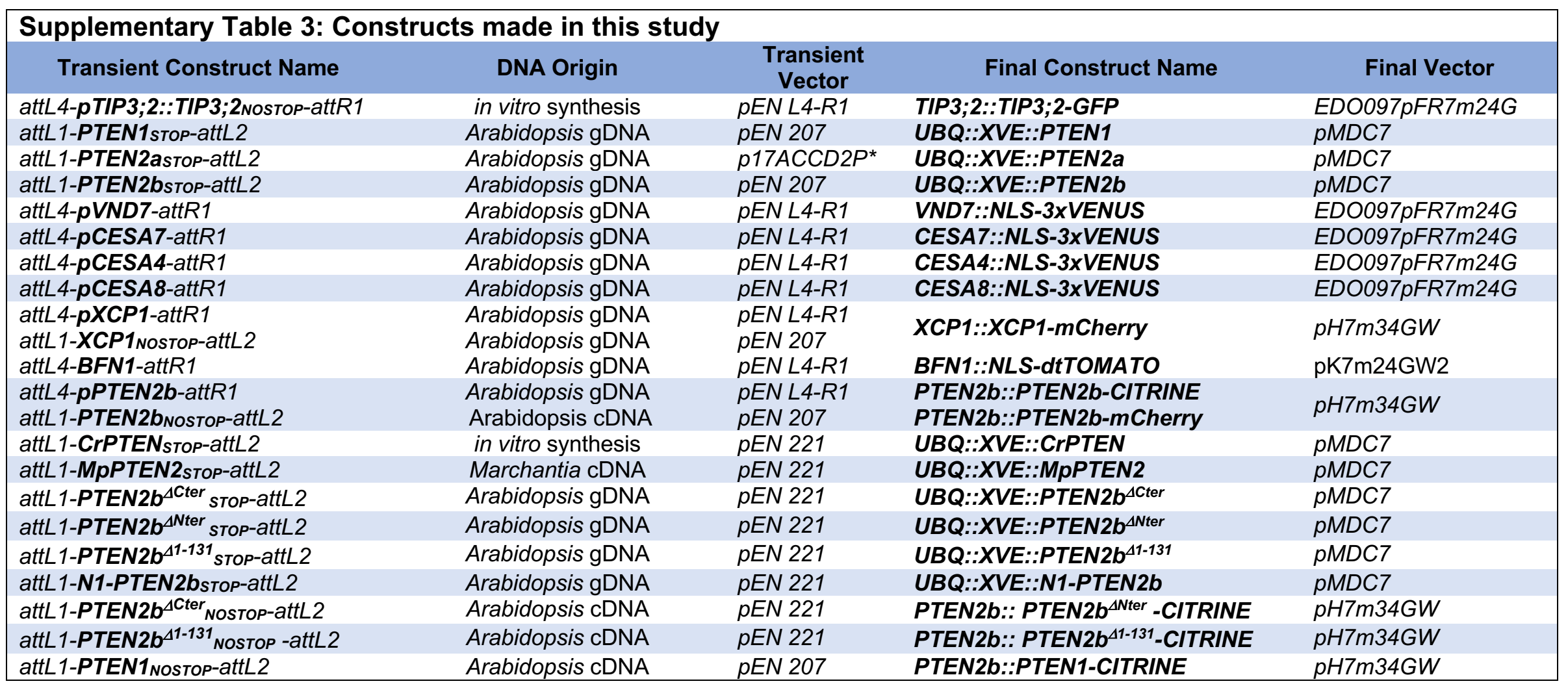

* p17ACCD2P was in vitro synthesized in pMk-RQ plasmid (Invitrogen) and contain attL1-MCS**-attL2 sequences.

**MCS: 5' - GAA TTC GAA GCT CGG TAC CCG GGG ATC CTC TAG AGT CGA CCT GCA GGC CCA TGG TGA CTA GTC AAG CTT-3' 